Supporting Information for

\title{
Biscyclization Reactions in Butadiyne- and Ethyne-linked Triazenes and Diazenes: Concerted vs. Stepwise Cyclizations
}

\author{
Laura D. Shirtcliff, Austin G. Hayes, and Michael M. Haley* \\ Department of Chemistry, University of Oregon, Eugene, Oregon 97403-1253 \\ haley@uoregon.edu
}

Felix Köhler, Kirsten Hess, and Rainer Herges*

Institut für Organische Chemie, Universität Kiel, 24098 Kiel, Germany

rherges@oc.uni-kiel.de

Table of Contents

Page

$\begin{array}{ll}\text { Experimental procedures } & \text { S2 }\end{array}$

$\begin{array}{ll}\text { Complete citation for Reference 32 } & \text { S7 }\end{array}$

$\begin{array}{ll}\text { Figure S1 S8 } & \text { S1 }\end{array}$

Figure S2 $\quad$ S9

$\begin{array}{ll}\text { NMR spectra } & \text { S10 }\end{array}$

$\begin{array}{ll}\text { X-ray data for } \mathbf{1 2} & \text { S18 }\end{array}$

$\begin{array}{lr}\text { Cartesian coordinates of calculated structures } & \text { S26 }\end{array}$ 


\section{Experimental Section}

General. ${ }^{1} \mathrm{H}$ and ${ }^{13} \mathrm{C}$ NMR spectra were recorded on a $300 \mathrm{MHz}$ spectrometer $(1 \mathrm{H}$, 299.95 MHz; $\left.{ }^{13} \mathrm{C}, 75.43 \mathrm{MHz}\right)$. Chemical shifts $(\delta)$ are expressed in ppm downfield from $\mathrm{SiMe}_{4}$ using the residual $\mathrm{CHCl}_{3}$ as internal standard $\left(\mathrm{CDCl}_{3}:{ }^{1} \mathrm{H}, 7.26 \mathrm{ppm} ;{ }^{13} \mathrm{C}, 77.0 \mathrm{ppm}\right)$. Coupling constants are expressed in hertz. IR spectra were recorded using an FTIR spectrometer. Mass spectra were recorded using an LC mass spectrometer with either APCI or ESI sources. Melting points are uncorrected. $\mathrm{CH}_{2} \mathrm{Cl}_{2}$ and $\mathrm{Et}_{3} \mathrm{~N}$ were distilled from $\mathrm{CaH}_{2}$ under a $\mathrm{N}_{2}$ atmosphere prior to use. $\mathrm{THF}$ and $\mathrm{Et}_{2} \mathrm{O}$ were distilled from $\mathrm{Na} /$ benzophenone ketal under a $\mathrm{N}_{2}$ atmosphere prior to use. All other chemicals were of reagent grade quality and used as obtained from manufacturers. Reactions were carried out in an inert atmosphere (dry $\mathrm{N}_{2}$ or Ar) when necessary. Column chromatography was performed on reagent grade silica gel (230-400 mesh). Preparative and analytical thin-layer chromatography was performed on precoated silica gel plates.

General Procedure A: Cu-catalyzed Homocoupling. To a heated solution of alkyne of pyridine or pyridine/MeOH (1:1, ca. $0.1 \mathrm{M})$ was added $\mathrm{Cu}(\mathrm{OAc})_{2}(5$ eq. $)$. The mixture was stirred at $50{ }^{\circ} \mathrm{C}$ for ca. 2-12 h (depending on functional group) and the solvent was evaporated. The residue was taken into $5 \% \mathrm{Et}_{3} \mathrm{~N}$ in $\mathrm{CH}_{2} \mathrm{Cl}_{2}$ and vacuum filtered through silica. After solvent evaporation, purification by column chromatography gave the desired pure product.

H Dimer 5a. Alkyne 1a $(0.33 \mathrm{~g}, 1.6 \mathrm{mmol})$ was reacted with $\mathrm{Cu}(\mathrm{OAc})_{2}(2.7 \mathrm{~g}, 14 \mathrm{mmol})$ in pyridine $(20 \mathrm{~mL})$ according to General Procedure A. Purification by column chromatography $\left(2.5 \% \mathrm{Et}_{3} \mathrm{~N}\right.$ in 1:5 $\mathrm{CH}_{2} \mathrm{Cl}_{2}$ :hexanes) afforded dimer $\mathbf{5 a}(0.32 \mathrm{~g}, 98 \%)$ as a light yellow powder: mp 103.9-104.4 ${ }^{\circ} \mathrm{C} ;{ }^{1} \mathrm{H} \mathrm{NMR}\left(\mathrm{CDCl}_{3}\right) \delta 7.52(\mathrm{~d}, J=7.5 \mathrm{~Hz}, 2 \mathrm{H}), 7.43$ $(\mathrm{d}, J=8.4 \mathrm{~Hz}, 2 \mathrm{H}), 7.30(\mathrm{t}, J=7.2 \mathrm{~Hz}, 2 \mathrm{H}), 7.08(\mathrm{t}, J=7.8 \mathrm{~Hz}, 2 \mathrm{H}), 3.81(\mathrm{q}, J=7.2 \mathrm{~Hz} 8 \mathrm{H})$, $1.33(\mathrm{t}, J=7.2 \mathrm{~Hz}, 12 \mathrm{H}) ;{ }^{13} \mathrm{C} \mathrm{NMR}\left(\mathrm{CDCl}_{3}\right) \delta 153.8,133.6,129.3,124.5,117.1,116.9,80.5$, 78.1, 48.9 (br), 41.8 (br), 14.4 (br), 10.8 (br); IR (neat) 3295, 3063, 2975, 2934, 2872, 2208, $2142 \mathrm{~cm}^{-1}$; MS (APCI) $\mathrm{m} / z(\%) 401.2\left(100, \mathrm{M}^{+}+\mathrm{H}\right), 328.3\left(25, \mathrm{M}^{+}-\mathrm{C}_{4} \mathrm{H}_{10} \mathrm{~N}\right), 300.3\left(45, \mathrm{M}^{+}-\right.$ $\mathrm{C}_{4} \mathrm{H}_{10} \mathrm{~N}_{3}$ ).

t-Butyl Dimer 5b. Alkyne 1b $(0.31 \mathrm{~g}, 1.2 \mathrm{mmol})$ was reacted with $\mathrm{Cu}(\mathrm{OAc})_{2}(2.7 \mathrm{~g}, 14$ $\mathrm{mmol})$ in pyridine $(20 \mathrm{~mL})$ according to General Procedure A. Purification by column chromatography $\left(2.5 \% \mathrm{Et}_{3} \mathrm{~N}\right.$ in $1: 5 \mathrm{CH}_{2} \mathrm{Cl}_{2}$ :hexanes) afforded dimer $\mathbf{5 b}(0.30 \mathrm{~g}, 99 \%)$ as a light yellow powder: $\mathrm{mp} 110.1-110.9{ }^{\circ} \mathrm{C} ;{ }^{1} \mathrm{H} \mathrm{NMR}\left(\mathrm{CDCl}_{3}\right) \delta$ 7.53-7.52 (m, 2H), 7.32-7.31 $(\mathrm{m}, 4 \mathrm{H}), 3.79(\mathrm{q}, J=7.5 \mathrm{~Hz}, 8 \mathrm{H}), 1.32(\mathrm{~s}, 18 \mathrm{H}), 1.31(\mathrm{t}, J=7.5 \mathrm{~Hz}, 12 \mathrm{H}) ;{ }^{13} \mathrm{C} \mathrm{NMR}\left(\mathrm{CDCl}_{3}\right)$ 
$\delta$ 151.5, 147.5, 130.4, 126.9, 116.8, 116.4, 81.0, 77.6, 34.3, 31.2, 31.11, 31.10; IR (neat) 3063, 2964, 2870, 2209, 2139, $1728 \mathrm{~cm}^{-1}$; MS (APCI) $\mathrm{m} / z$ (\%) $513.3\left(100, \mathrm{M}^{+}+\mathrm{H}\right), 440.3$ $\left(25, \mathrm{M}^{+}-\mathrm{C}_{4} \mathrm{H}_{10} \mathrm{~N}\right), 412.3\left(45, \mathrm{M}^{+}-\mathrm{C}_{4} \mathrm{H}_{10} \mathrm{~N}_{3}\right)$.

Chloro Dimer 5c. Alkyne 1c $(0.32 \mathrm{~g}, 1.3 \mathrm{mmol})$ was reacted with $\mathrm{Cu}(\mathrm{OAc})_{2}(2.7 \mathrm{~g}, 14$ $\mathrm{mmol})$ in pyridine $(20 \mathrm{~mL})$ according to General Procedure A. Purification by column chromatography (2.5\% $\mathrm{Et}_{3} \mathrm{~N}$ in 1:5 $\mathrm{CH}_{2} \mathrm{Cl}_{2}$ :hexanes) afforded dimer $\mathbf{5 c}(0.30 \mathrm{~g}, 96 \%)$ as a light yellow powder: mp 107.3-107.7 ${ }^{\circ} \mathrm{C} ;{ }^{1} \mathrm{H} \mathrm{NMR}\left(\mathrm{CDCl}_{3}\right) \delta 7.46(\mathrm{~d}, J=2.4 \mathrm{~Hz}, 2 \mathrm{H}), 7.36$ (d, $J=8.5 \mathrm{~Hz}, 2 \mathrm{H}), 7.22$ (dd, $J=8.5,2.4 \mathrm{~Hz}, 2 \mathrm{H}), 3.80$ (q, $J=7.2 \mathrm{~Hz} 8 \mathrm{H}), 1.31$ (br s, $12 \mathrm{H})$; ${ }^{13} \mathrm{C} \mathrm{NMR}\left(\mathrm{CDCl}_{3}\right) \delta$ 152.5, 132.8, 129.7, 129.4, 118.3, 118.1, 79.67, 78.8, 49.2 (br), 42.0 (br), 14.4 (br), 10.8 (br); IR (neat) 3067, 2972, 2936, 2873, 2202, 2135, 1892, $1587 \mathrm{~cm}^{-1}$; MS (APCI) $m / z(\%) 469.2\left(100, \mathrm{M}^{+}+\mathrm{H}\right), 396.1\left(70, \mathrm{M}^{+}-\mathrm{C}_{4} \mathrm{H}_{10} \mathrm{~N}\right), 368.1\left(45, \mathrm{M}^{+}-\mathrm{C}_{4} \mathrm{H}_{10} \mathrm{~N}_{3}\right) 269.0$ $\left(20, \mathrm{M}^{+}-\mathrm{C}_{8} \mathrm{H}_{20} \mathrm{~N}_{6}\right)$.

Nitro Dimer 5d. Alkyne 1d $(0.30 \mathrm{~g}, 1.2 \mathrm{mmol})$ was reacted with $\mathrm{Cu}(\mathrm{OAc})_{2}(2.7 \mathrm{~g}, 14$ $\mathrm{mmol})$ in pyridine $(20 \mathrm{~mL})$ according to General Procedure A. Purification by column chromatography (2.5\% $\mathrm{Et}_{3} \mathrm{~N}$ in 1:5 $\mathrm{CH}_{2} \mathrm{Cl}_{2}$ :hexanes) afforded dimer $\mathbf{5 d}(0.29 \mathrm{~g}, 96 \%)$ as a slightly yellow powder: $\mathrm{mp} 166.6-167.2{ }^{\circ} \mathrm{C} ;{ }^{1} \mathrm{H} \mathrm{NMR}\left(\mathrm{CDCl}_{3}\right) \delta 8.37(\mathrm{~d}, J=2.7 \mathrm{~Hz}, 2 \mathrm{H})$, $8.12(\mathrm{dd}, J=9.0,2.7 \mathrm{~Hz}, 2 \mathrm{H}), 7.55(\mathrm{~d}, J=9.0 \mathrm{~Hz}, 2 \mathrm{H}), 3.91(\mathrm{q}, J=7.2 \mathrm{~Hz}, 4 \mathrm{H}), 3.89(\mathrm{q}, J=$ $7.1 \mathrm{~Hz} 4 \mathrm{H}), 1.41(\mathrm{t}, J=7.2 \mathrm{~Hz}, 6 \mathrm{H}), 1.35(\mathrm{t}, J=7.2 \mathrm{~Hz}, 6 \mathrm{H}) ;{ }^{13} \mathrm{C} \mathrm{NMR}\left(\mathrm{CDCl}_{3}\right) \delta$ 158.4, 143.8, 129.4, 124.8, 121.7, 117.2, , 79.2, 79.1, 50.1, 43.0, 14.3, 10.7; IR (neat) 2978, 2933, $1598,1571,1508,1465 \mathrm{~cm}^{-1}$; MS (APCI) $\mathrm{m} / z(\%) 491.2\left(100 \mathrm{M}^{+}+\mathrm{H}\right), 418.0\left(25, \mathrm{M}^{+}-\right.$ $\left.\mathrm{C}_{4} \mathrm{H}_{10} \mathrm{~N}\right), 390.0\left(45, \mathrm{M}^{+}-\mathrm{C}_{4} \mathrm{H}_{10} \mathrm{~N}_{3}\right)$.

Cyano Dimer 5e. Alkyne 1e $(0.25 \mathrm{~g}, 1.1 \mathrm{mmol})$ was reacted with $\mathrm{Cu}(\mathrm{OAc})_{2}(1.1 \mathrm{~g}, 5.5$ $\mathrm{mmol})$ in pyridine $(10 \mathrm{~mL})$ according to General Procedure A. Filtration over a short pad of silica (washing through $\left.\mathrm{CH}_{2} \mathrm{Cl}_{2}\right)$ afforded dimer $5 \mathbf{e}(0.24 \mathrm{~g}, 98 \%)$ as an yellow-orange powder: mp 145.2-146.2 ${ }^{\circ} \mathrm{C} .{ }^{1} \mathrm{H}$ NMR $\left(\mathrm{CDCl}_{3}\right) \delta 7.76(\mathrm{t}, J=1.2 \mathrm{~Hz}, 2 \mathrm{H}), 7.51-7.50(\mathrm{~m}, 4 \mathrm{H})$, $3.86(\mathrm{q}, J=7.0 \mathrm{~Hz}, 8 \mathrm{H}), 1.38(\mathrm{t}, J=7.0 \mathrm{~Hz}, 6 \mathrm{H}), 1.31(\mathrm{t}, J=7.1 \mathrm{~Hz}, 6 \mathrm{H}) ;{ }^{13} \mathrm{C} \mathrm{NMR}\left(\mathrm{CDCl}_{3}\right)$

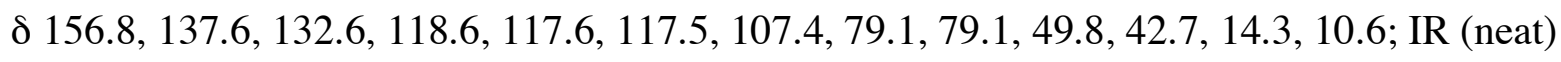
$3068,2978,2936,2874,2580,2532,2225,1917 \mathrm{~cm}^{-1}$; MS (APCI) $\mathrm{m} / \mathrm{z}$ (\%) 451.2 (100, $\left.\mathrm{M}^{+}+\mathrm{H}\right) 473.2\left(40, \mathrm{M}^{+}+\mathrm{Na}\right)$.

Methoxy Dimer 5f. Alkyne 1f $(0.50 \mathrm{~g}, 2.2 \mathrm{mmol})$ was reacted with $\mathrm{Cu}(\mathrm{OAc})_{2}(2.15 \mathrm{~g}$, $10.8 \mathrm{mmol})$ in pyridine/ $\mathrm{MeOH}(1: 1,40 \mathrm{~mL})$ according to General Procedure A. Purification by column chromatography (2.5\% $\mathrm{Et}_{3} \mathrm{~N}$ in 1:1:5 EtOAc: $\mathrm{CH}_{2} \mathrm{Cl}_{2}$ :hexanes) afforded dimer $\mathbf{5 f}$ $(0.44 \mathrm{~g}, 87 \%)$ as an orange-yellow powder: $\mathrm{mp} 113.2-113.8^{\circ} \mathrm{C} .{ }^{1} \mathrm{H} \mathrm{NMR}\left(\mathrm{CDCl}_{3}\right) \delta 7.35(\mathrm{~d}$, 
$J=9.1 \mathrm{~Hz}, 2 \mathrm{H}), 7.01(\mathrm{~d}, J=2.9 \mathrm{~Hz}, 2 \mathrm{H}), 6.87(\mathrm{dd}, J=9.1,2.9 \mathrm{~Hz}, 2 \mathrm{H}), 3.79(\mathrm{~s}, 6 \mathrm{H}), 3.77(\mathrm{q}$, $J=7.0 \mathrm{~Hz}, 8 \mathrm{H}), 1.30(\mathrm{t}, J=7.0 \mathrm{~Hz}, 12 \mathrm{H}) ;{ }^{13} \mathrm{C} \mathrm{NMR}\left(\mathrm{CDCl}_{3}\right) \delta 156.8,148.4,118.4,117.7$, 117.3 116.9, , 80.8, 78.4, 55.8, 52.8, 12.4; IR (neat) 3077, 2979, 2932, 2870, 2830, 2209, 2132, 1736, $1594 \mathrm{~cm}^{-1}$; MS (APCI) $\mathrm{m} / z(\%) 461.0\left(100, \mathrm{M}^{+}+\mathrm{H}\right), 388.0\left(85, \mathrm{M}^{+}-\mathrm{C}_{4} \mathrm{H}_{10} \mathrm{~N}\right)$.

General Procedure B: Biscyclization. A solution of dimer in ODCB (ca. $0.2 \mathrm{M}$ ) was heated to $90{ }^{\circ} \mathrm{C}$ for $2-8 \mathrm{~h}$ under $\mathrm{N}_{2}$. The reactions were monitored by TLC. Upon completion, the solution was cooled and diluted with hexanes. The mixture filtered over a short pad of silica, first washing with hexanes (to remove ODCB), then eluting with 1:1 $\mathrm{CH}_{2} \mathrm{Cl}_{2}$ :EtOAc. The solvent was evaporated and purification by preparative TLC gave the desired pure product.

H Cyclized Dimer 6a. A solution of dimer 5a (95 mg, $0.24 \mathrm{mmol})$ in ODCB (10 mL) was heated to $90{ }^{\circ} \mathrm{C}$ according to General Procedure B. Purification by preparative TLC (45\% hexanes $/ \mathrm{CH}_{2} \mathrm{Cl}_{2}$ ) provided $6 \mathbf{a}\left(93 \mathrm{mg}, 98 \%\right.$ ) as an orange powder: $\mathrm{mp} 170.5-171.8^{\circ} \mathrm{C}$; ${ }^{1} \mathrm{H} \mathrm{NMR}\left(\mathrm{CDCl}_{3}\right) \delta 7.80(\mathrm{~d}, J=8.3 \mathrm{~Hz}, 2 \mathrm{H}), 7.77(\mathrm{~d}, J=8.7 \mathrm{~Hz}, 2 \mathrm{H}), 7.40-7.35(\mathrm{~m}, 2 \mathrm{H})$, 7.26-7.20 (m, 2H), 3.38 (q, $J=7.3 \mathrm{~Hz} 8 \mathrm{H}), 0.99(\mathrm{t}, J=7.3 \mathrm{~Hz}, 12 \mathrm{H}) ;{ }^{13} \mathrm{C} \mathrm{NMR}\left(\mathrm{CDCl}_{3}\right) \delta$ $146.2,126.7,122.9,121.8,120.1,118.0$, there aren't any others, 87.1, 52.5, 12.1; IR (neat) 3054, 2973, $22091466 \mathrm{~cm}^{-1}$; MS (APCI) $\mathrm{m} / z$ (\%) $401.4\left(100, \mathrm{M}^{+}+\mathrm{H}\right), 329.2\left(25, \mathrm{M}^{+}-\right.$ $\left.\mathrm{C}_{4} \mathrm{H}_{10} \mathrm{~N}\right), 314.2\left(25, \mathrm{M}^{+}-\mathrm{C}_{4} \mathrm{H}_{10} \mathrm{~N}_{2}\right), 300.2\left(45, \mathrm{M}^{+}-\mathrm{C}_{4} \mathrm{H}_{10} \mathrm{~N}_{3}\right)$.

$\boldsymbol{t}$-Butyl Cyclized Dimer 6b. A solution of dimer $5 \mathbf{b}(85 \mathrm{mg}, 0.17 \mathrm{mmol})$ in ODCB (10 $\mathrm{mL}$ ) was reacted according to General Procedure B. Purification by preparative TLC (55\% $\mathrm{CH}_{2} \mathrm{Cl}_{2} /$ hexanes) provided $\mathbf{6 c}(84 \mathrm{mg}, 99 \%)$ as an orange powder: $\mathrm{mp} 137.5-138.4{ }^{\circ} \mathrm{C} ;{ }^{1} \mathrm{H}$ NMR $\left(\mathrm{CDCl}_{3}\right) \delta 7.71(\mathrm{~d}, J=2.1 \mathrm{~Hz}, 2 \mathrm{H}), 7.69(\mathrm{~d}, J=8.4 \mathrm{~Hz}, 2 \mathrm{H}), 7.49(\mathrm{dd}, J=8.4,2.1 \mathrm{~Hz}$, $2 \mathrm{H}), 3.36(\mathrm{q}, J=6.9 \mathrm{~Hz}, 8 \mathrm{H}), 1.41(\mathrm{~s}, 18 \mathrm{H}), 0.99(\mathrm{t}, J=6.9 \mathrm{~Hz}, 12 \mathrm{H}) ;{ }^{13} \mathrm{C} \mathrm{NMR}\left(\mathrm{CDCl}_{3}\right) \delta$ $145.8,145.1,126.5,121.8,120.2,117.6,114.4,87.4,52.6,35.0,31.2,12.2$; IR (neat) 2968, 2869, 2208, 1906, 1730, $1465 \mathrm{~cm}^{-1}$; MS (APCI) $\mathrm{m} / \mathrm{z}(\%) 513.3\left(100, \mathrm{M}^{+}+\mathrm{H}\right), 441.2\left(50, \mathrm{M}^{+}-\right.$ $\left.\mathrm{C}_{4} \mathrm{H}_{9} \mathrm{~N}\right), 398.3\left(15, \mathrm{M}^{+}-2 \mathrm{C}_{4} \mathrm{H}_{9}\right)$.

Chloro Cyclized Dimer 6c. A solution of dimer 5c (91 mg, $0.19 \mathrm{mmol})$ in ODCB (10 $\mathrm{mL}$ ) was reacted according to General Procedure B. Purification by preparative TLC (45\% hexanes in $\mathrm{CH}_{2} \mathrm{Cl}_{2}$ ) provided $\mathbf{6 c}(88 \mathrm{mg}, 97 \%)$ as an orange powder: $\mathrm{mp} 104.9-106.0{ }^{\circ} \mathrm{C} ;{ }^{1} \mathrm{H}$ $\operatorname{NMR}\left(\mathrm{CDCl}_{3}\right) \delta 7.76(\mathrm{~d}, J=2.1 \mathrm{~Hz}, 2 \mathrm{H}), 7.68(\mathrm{~d}, J=9.0 \mathrm{~Hz}, 2 \mathrm{H}), 7.31(\mathrm{dd}, J=9.0,2.1 \mathrm{~Hz}$, 2H), 3.35 (q, $J=7.1 \mathrm{~Hz}, 8 \mathrm{H}), 0.97(\mathrm{t}, J=7.1 \mathrm{~Hz}, 12 \mathrm{H}) ;{ }^{13} \mathrm{C} \mathrm{NMR}\left(\mathrm{CDCl}_{3}\right) \delta$ 144.6, 129.0, 128.3, 122.2, 119.8, 119.7, 119.0, 87.0, 52.6, 12.1; IR (neat) 3064, 2976, 2936, 2865, 2211, $1895,1727 \mathrm{~cm}^{-1}$; MS (APCI) $\mathrm{m} / z(\%) 469.2\left(100, \mathrm{M}^{+}+\mathrm{H}\right)$. 
Nitro Cyclized Dimer 6d. A solution of dimer 5d (92 mg, $0.19 \mathrm{mmol}$ ) in ODCB (10 $\mathrm{mL}$ ) was reacted according to General Procedure B. Purification by preparative TLC (45\% hexanes in $\mathrm{CH}_{2} \mathrm{Cl}_{2}$ ) provided $\mathbf{6 d}(89 \mathrm{mg}, 97 \%)$ as an orange powder: $\mathrm{mp} 221.2-222.3{ }^{\circ} \mathrm{C} ;{ }^{1} \mathrm{H}$ NMR $\left(\mathrm{CDCl}_{3}\right) \delta 8.84(\mathrm{~d}, J=2.1 \mathrm{~Hz}, 2 \mathrm{H}), 8.16(\mathrm{dd}, J=9.3,2.1 \mathrm{~Hz}, 2 \mathrm{H}), 7.80(\mathrm{~d}, J=9.3 \mathrm{~Hz}$, 2H), $3.40(\mathrm{q}, J=6.9 \mathrm{~Hz} 8 \mathrm{H}), 0.98(\mathrm{t}, J=6.9 \mathrm{~Hz}, 12 \mathrm{H}) ;{ }^{13} \mathrm{C} \mathrm{NMR}\left(\mathrm{CDCl}_{3}\right) \delta 147.3,143.9$, 123.5, 121.1, 120.2, 119.2, 118.9, 87.9, 52.7, 12.0; IR (KBr) 2984, 2939, $1626 \mathrm{~cm}^{-1}$; MS (APCI) $m / z(\%) 491.3\left(100, \mathrm{M}^{+}+\mathrm{H}\right), 418.9\left(\mathrm{M}^{+}-\mathrm{C}_{4} \mathrm{H}_{10} \mathrm{~N}_{2} \mathrm{O}_{2}\right) 390.0$.

Cyano Cyclized Dimer 6e. A solution of dimer 5 e $(98 \mathrm{mg}, 0.22 \mathrm{mmol})$ in ODCB (10 $\mathrm{mL}$ ) was reacted according to General Procedure B. Purification by preparative TLC (45\% hexanes in $\mathrm{CH}_{2} \mathrm{Cl}_{2}$ ) provided $6 \mathbf{e}\left(96 \mathrm{mg}\right.$, 98\%) as an orange powder: $\mathrm{mp} 179.7-181.0{ }^{\circ} \mathrm{C} ;{ }^{1} \mathrm{H}$ $\operatorname{NMR}\left(\mathrm{CDCl}_{3}\right) \delta 8.21(\mathrm{~d}, J=1.9 \mathrm{~Hz}, 2 \mathrm{H}), 7.79(\mathrm{dd}, J=8.7,1.9 \mathrm{~Hz}, 2 \mathrm{H}), 7.48(\mathrm{~d}, J=8.7 \mathrm{~Hz}$, $2 \mathrm{H}), 3.36(\mathrm{q}, J=6.9 \mathrm{~Hz}, 8 \mathrm{H}), 0.94(\mathrm{t}, J=6.9 \mathrm{~Hz}, 12 \mathrm{H}) ;{ }^{13} \mathrm{C} \mathrm{NMR}\left(\mathrm{CDCl}_{3}\right) \delta 146.2,127.5$, 127.5, 121.3, 120.7, 119.5, 119.3, 106.5, 87.2, 52.6, 11.9; IR (KBr) 2977, 2221, 2166, 1622 $\mathrm{cm}^{-1}$; MS (APCI) $m / z(\%) 469.2\left(100, \mathrm{M}^{+}+\mathrm{H}\right)$.

Methoxy Cyclized Dimer 6f. Alkyne dimer $5 f(30 \mathrm{mg}, 0.07 \mathrm{mmol})$ in ODCB (3mL) was reacted according to general procedure $\mathrm{B}\left(100^{\circ} \mathrm{C}, 15 \mathrm{~h}\right)$. After concentration the crude product was purified by preparative chromatography $\left(1: 1: 4 \mathrm{CH}_{2} \mathrm{Cl}_{2}\right.$ :EtOAc:hexanes) to afford bisisoindazole $\mathbf{6 f}(27 \mathrm{mg}, 87 \%)$ as a yellow-orange solid: $\mathrm{mp} 133.2-133.8{ }^{\circ} \mathrm{C} ;{ }^{1} \mathrm{H}$ NMR $\left(\mathrm{CDCl}_{3}\right) \delta 7.64(\mathrm{~d}, J=9.4 \mathrm{~Hz}, 2 \mathrm{H}), 7.06(\mathrm{dd}, J=9.4,2.4 \mathrm{~Hz}, 2 \mathrm{H}), 7.00(\mathrm{~d}, J=2.4 \mathrm{~Hz}, 2 \mathrm{H})$, 3.88 (s, 6H), 3.33 (q, $J=7.0 \mathrm{~Hz}, 8 \mathrm{H}), 0.98(\mathrm{t}, J=7.0 \mathrm{~Hz}, 12 \mathrm{H}) ;{ }^{13} \mathrm{C} \mathrm{NMR}\left(\mathrm{CDCl}_{3}\right) \delta 156.4$, 143.0, 122.3, 121.7, 119.8, 97.0, 87.5, 55.6, 52.8, 12.4; IR (KBr) 2965, 2054, 1635, $1218 \mathrm{~cm}^{-}$ ${ }^{1}$; MS (APCI) $m / z(\%) 461.2\left(100, \mathrm{M}^{+}+\mathrm{H}\right), 389.1\left(40, \mathrm{M}^{+}-\mathrm{C}_{4} \mathrm{H}_{10} \mathrm{~N}\right), 374.0\left(20, \mathrm{M}^{+}-\mathrm{C}_{4} \mathrm{H}_{10} \mathrm{~N}_{2}\right)$.

Pd-catalyzed Methoxy Cyclized Dimer 6f. Triazene $5 \mathbf{f}(0.35 \mathrm{~g}, 1.53 \mathrm{mmol})$ was dissolved in a minimal amount of THF and DIPA $(1: 1,15 \mathrm{~mL}) . \mathrm{PdCl}_{2}\left(\mathrm{PPh}_{3}\right)_{2}(54 \mathrm{mg}, 0.08$ $\mathrm{mmol}), \mathrm{CuI}(29 \mathrm{mg}, 0.15 \mathrm{mmol})$, and $\mathrm{I}_{2}(0.196 \mathrm{~g}, 0.77 \mathrm{mmol})$ were added and the reaction was placed in a $50{ }^{\circ} \mathrm{C}$ sand bath for $1.5 \mathrm{~h}$. The crude reaction mixture was concentrated in vacuo and then dissolved in 1:1 $\mathrm{CH}_{2} \mathrm{Cl}_{2} /$ hexanes, filtered over a short pad of silica and concentrated in vacuo to yield dimer $\mathbf{6 f}(0.35 \mathrm{~g}, 99 \%)$ as a orange-red solid. Spectral data were consistent with those described above.

$\boldsymbol{t}$-Butyl Piperidyl-cyclized Dimer 8. Triazene $7(0.52 \mathrm{~g}, 1.92 \mathrm{mmol})$ was dissolved in a minimal amount of THF and DIPA (1:1, $10 \mathrm{~mL}) . \mathrm{PdCl}_{2}\left(\mathrm{PPh}_{3}\right)_{2}(90 \mathrm{mg}, 0.134 \mathrm{mmol}), \mathrm{CuI}$ (50 $\mathrm{mg}, 0.27 \mathrm{mmol})$, and $\mathrm{I}_{2}(0.234 \mathrm{~g}, 0.96 \mathrm{mmol})$ were added and the reaction was placed in a $50{ }^{\circ} \mathrm{C}$ sand bath for $1.5 \mathrm{~h}$. The crude reaction mixture was concentrated in vacuo and then 
dissolved in 1:1 $\mathrm{CH}_{2} \mathrm{Cl}_{2} /$ hexanes, filtered over a short pad of silica and concentrated in vacuo to yield dimer $8(0.495 \mathrm{~g}, 97 \%)$ as a reddish solid: $\mathrm{mp}(\mathrm{dec}) 258.0{ }^{\circ} \mathrm{C} ;{ }^{1} \mathrm{H} \mathrm{NMR}\left(\mathrm{CDCl}_{3}\right) \delta$ 7.77-7.71 (m, 4H), $7.49(\mathrm{dd}, J=9.0,1.8 \mathrm{~Hz}, 2 \mathrm{H}), 3.40(\mathrm{t}, J=5.7 \mathrm{~Hz}, 8 \mathrm{H}), 1.94(\mathrm{q}, J=5.7$ $\mathrm{Hz}, 8 \mathrm{H}), 1.64(\mathrm{q}, J=5.7 \mathrm{~Hz}, 4 \mathrm{H}), 1.42(\mathrm{~s}, 18 \mathrm{H}) ;{ }^{13} \mathrm{C} \mathrm{NMR}\left(\mathrm{CDCl}_{3}\right) \delta 145.8,144.3,135.2$, 126.3, 122.1, 117.6, 114.1, 87.8, 56.4, 34.9, 31.2, 26.2, 23.5; IR (KBr) 2947, 2220, $1358 \mathrm{~cm}^{-}$ ${ }^{1}$; MS (APCI) $m / z(\%) 537.5\left(100, \mathrm{M}^{+}+\mathrm{H}\right), 452.1\left(30, \mathrm{M}^{+}-\mathrm{C}_{5} \mathrm{H}_{10} \mathrm{~N}\right), 424.1\left(40, \mathrm{M}^{+}-\mathrm{C}_{5} \mathrm{H}_{10} \mathrm{~N}_{3}\right)$.

Ethyne-linked Chloro Dimer 9. Alkyne 1c (1.50 g, $3.36 \mathrm{mmol})$ and iodide 10 (1.65 g, $4.89 \mathrm{mmol})$ were dissolved in THF and DIPA $(1: 1,30 \mathrm{~mL})$. The solution was purged with Ar for $15 \mathrm{~min}$, then $\mathrm{PdCl}_{2}$ (dppf) (0.20 g, $\left.0.25 \mathrm{mmol}\right)$ and $\mathrm{CuI}(93 \mathrm{mg}, 0.49 \mathrm{mmol})$ were added. The reaction was placed in a $50{ }^{\circ} \mathrm{C}$ sand bath for $16 \mathrm{~h}$. The crude product was concentrated in vacuo, taken up in $1: 1 \mathrm{CH}_{2} \mathrm{Cl}_{2} /$ hexanes, and filtered over a short pad of silica. After concentration the crude product was chromatographed on silica gel (4:1 hexanes $\left./ \mathrm{CH}_{2} \mathrm{Cl}_{2}\right)$ to afford $9(1.32 \mathrm{~g}, 62 \%)$ as a light yellow solid: $\mathrm{mp} 107.3-107.7{ }^{\circ} \mathrm{C} ;{ }^{1} \mathrm{H}$ $\operatorname{NMR}\left(\mathrm{CDCl}_{3}\right) \delta 7.44(\mathrm{~d}, J=2.1 \mathrm{~Hz}, 2 \mathrm{H}), 7.38(\mathrm{~d}, J=8.7 \mathrm{~Hz}, 2 \mathrm{H}), 7.20(\mathrm{dd}, J=8.7,2.1 \mathrm{~Hz}$, $2 \mathrm{H}), 3.82(\mathrm{q}, J=6.9 \mathrm{~Hz}, 8 \mathrm{H}), 1.33(\mathrm{t}, J=6.9 \mathrm{~Hz}, 12 \mathrm{H}) ;{ }^{13} \mathrm{C} \mathrm{NMR}\left(\mathrm{CDCl}_{3}\right) \delta 150.8,132.1$, 129.4, 128.8, 119.8, 116.0, 91.8, 49.2, 41.9, 14.6, 11.0; IR (KBr) 2966, 1924, 2017, $1465 \mathrm{~cm}^{-}$ ${ }^{1}$; MS (APCI) $m / z(\%) 445.1\left(100, \mathrm{M}^{+}+\mathrm{H}\right), 327.0(60)$.

Chloro bisisoindazole 11. A pressure bomb was charged with $9(0.20 \mathrm{~g}, 0.45 \mathrm{mmol})$, $\mathrm{CuCl}(10 \mathrm{mg}, 0.11 \mathrm{mmol})$ and $\mathrm{MeOH}(20 \mathrm{~mL})$ and placed in a $125^{\circ} \mathrm{C}$ sand bath for $16 \mathrm{~h}$ (CAUTION). The reaction was cooled to rt, diluted with EtOAc, filtered over a short pad of silica, and concentrated in vacuo to afford $11(0.19 \mathrm{~g}, 98 \%)$ as a yellowish powder: $\mathrm{mp}$ 170.5-171.8 ${ }^{\circ} \mathrm{C} ;{ }^{1} \mathrm{H} \mathrm{NMR}\left(\mathrm{CDCl}_{3}\right) \delta 7.72(\mathrm{~d}, J=8.7 \mathrm{~Hz}, 2 \mathrm{H}), 7.30(\mathrm{dd}, J=8.7,2.1 \mathrm{~Hz}, 2 \mathrm{H})$, $7.19(\mathrm{~d}, J=2.1 \mathrm{~Hz}, 2 \mathrm{H}), 3.28(\mathrm{q}, J=6.9 \mathrm{~Hz}, 4 \mathrm{H}), 0.87(\mathrm{t}, J=6.9 \mathrm{~Hz}, 12 \mathrm{H}) ;{ }^{13} \mathrm{C} \mathrm{NMR}$ $\left(\mathrm{CDCl}_{3}\right) \delta 144.3,128.5,127.9,122.8,121.7,119.5,118.4,51.6,11.9$; IR (KBr) 2976, 2934, 1646, $1474 \mathrm{~cm}^{-1}$; MS (APCI) $m / z(\%) 445.1\left(100, \mathrm{M}^{+}+\mathrm{H}\right), 374.0\left(80, \mathrm{M}^{+}-\mathrm{Cl}_{2}\right)$.

Rearranged Chloro Bisindazole 12. A pressure bomb was charged with diyne 5c $(0.166 \mathrm{~g}, 0.354 \mathrm{mmol})$ and $\mathrm{MeOH}(20 \mathrm{~mL})$ and placed in a $125^{\circ} \mathrm{C}$ sand bath for $10 \mathrm{~h}$ (CAUTION). The reaction was cooled to rt, diluted with $\mathrm{CH}_{2} \mathrm{Cl}_{2}$, filtered over a short pad of silica (washing with EtOAc), and concentrated in vacuo to yield the expected isoindazole 7c $(0.125 \mathrm{~g}, 75 \%)$. Spectral data were consistent with those described above. The silica pad was washed with $\mathrm{MeOH}$ and another fraction was collected to yield 12 (35 $\mathrm{mg}, 21 \%)$ as a yellow solid: $\mathrm{mp}(\mathrm{dec}) 307.7-307.9{ }^{\circ} \mathrm{C} ;{ }^{1} \mathrm{H} \mathrm{NMR}\left(\mathrm{CDCl}_{3}\right) \delta 7.95(\mathrm{~d}, J=8.7 \mathrm{~Hz}, 2 \mathrm{H}), 7.17$ (dd, $J=8.7,1.8 \mathrm{~Hz}, 2 \mathrm{H}), 6.94(\mathrm{~d}, J=1.8 \mathrm{~Hz}, 2 \mathrm{H}), 5.29(\mathrm{sext}, J=6.9 \mathrm{~Hz}, 2 \mathrm{H}), 4.50(\mathrm{sext}, J=6.9$ 
$\mathrm{Hz}, 2 \mathrm{H}), 3.55$ (sext, $J=6.9 \mathrm{~Hz}, 4 \mathrm{H}), 1.73(\mathrm{t}, J=6.9 \mathrm{~Hz}, 6 \mathrm{H}), 0.94(\mathrm{t}, J=6.9 \mathrm{~Hz}, 6 \mathrm{H}) ;{ }^{13} \mathrm{C}$

$\operatorname{NMR}\left(\mathrm{CDCl}_{3}\right) \delta 153.5,149.3,132.7,132.3,129.3$ 125.2, 120.7, 118.6, 49.1, 48.7, 12.6, 11.7;

IR (KBr) 2977, 2919, 1565, $1548 \mathrm{~cm}^{-1}$; MS (APCI) $\mathrm{m} / z$ (\%) $468.7\left(100, \mathrm{M}^{+}+\mathrm{H}\right)$

\section{Complete Citation for Reference 32}

Frisch, M. J.; Trucks, G. W.; Schlegel, H. B.; Scuseria, G. E.; Robb, M. A.; Cheeseman, J.

R.; Zakrzewski, V. G.; Montgomery, J. A., Jr.; Stratmann, R. E.; Burant, J. C.; Dapprich, S.;

Millam, J. M.; Daniels, A. D.; Kudin, K. N.; Strain, M. C.; Farkas, O.; Tomasi, J.; Barone,

V.; Cossi, M.; Cammi, R.; Mennucci, B.; Pomelli, C.; Adamo, C.; Clifford, S.; Ochterski, J.;

Petersson, G. A.; Ayala, P. Y.; Cui, Q.; Morokuma, K.; Malick, D. K.; Rabuck, A. D.;

Raghavachari, K.; Foresman, J. B.; Cioslowski, J.; Ortiz, J. V.; Stefanov, B. B.; Liu, G.;

Liashenko, A.; Piskorz, P.; Komaromi, I.; Gomperts, R.; Martin, R. L.; Fox, D. J.; Keith, T.;

Al-Laham, M. A.; Peng, C. Y.; Nanayakkara, A.; Gonzalez, C.; Challacombe, M.; Gill, P. M.

W.; Johnson, B.; Chen, W.; Wong, M. W.; Andres, J. L.; Gonzalez, C.; Head-Gordon, M.;

Replogle, E. S.; Pople, J. A.; Revision A.6 ed.; Gaussian: Pittsburg, PA, 1998. 

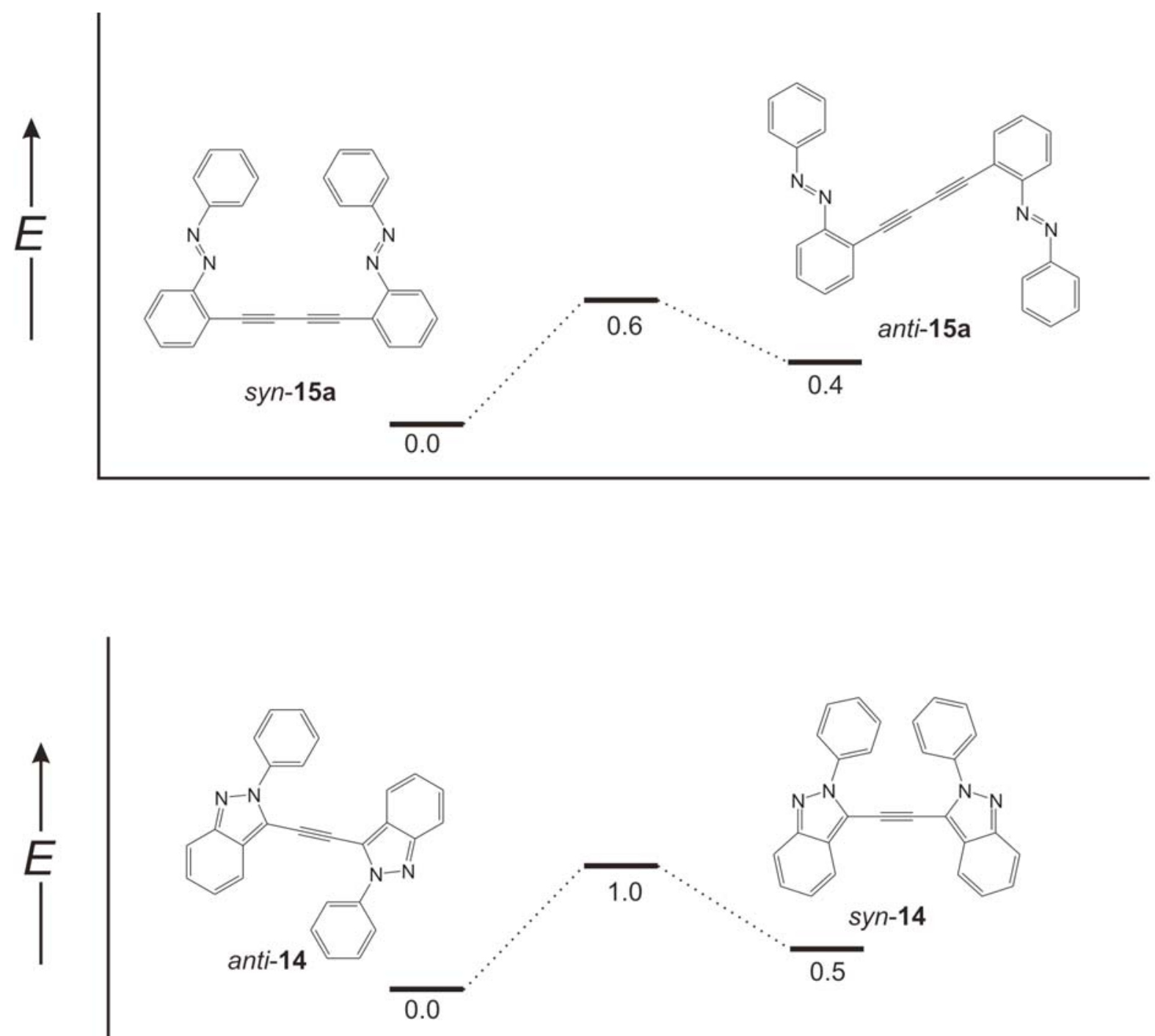

Figure S1. Rotational barriers of reactant and product of the diazene diyne. 


\begin{tabular}{ccc}
\hline structure & CIV & C-N Bond Length $(\AA)$ \\
\hline $\mathbf{1 8 b}$ & $0.052 / 0.025$ & $1.818 / 2.690$ \\
$\mathbf{2 0 b}$ & 0.037 & 2.294 \\
$\mathbf{1 8 c}$ & 0.057 & 1.804 \\
$\mathbf{2 3 b}$ & $0.045 / 0.025$ & $1.813 / 2.526$ \\
$\mathbf{2 3 c}$ & 0.054 & 1.726 \\
$\mathbf{2 7 b}$ & 0.027 & 2.100 \\
$\mathbf{2 7 c}$ & 0.050 & 1.849 \\
$\mathbf{3 1 b}$ & 0.037 & 2.111 \\
$\mathbf{3 1 c}$ & 0.042 & 1.773 \\
\hline
\end{tabular}

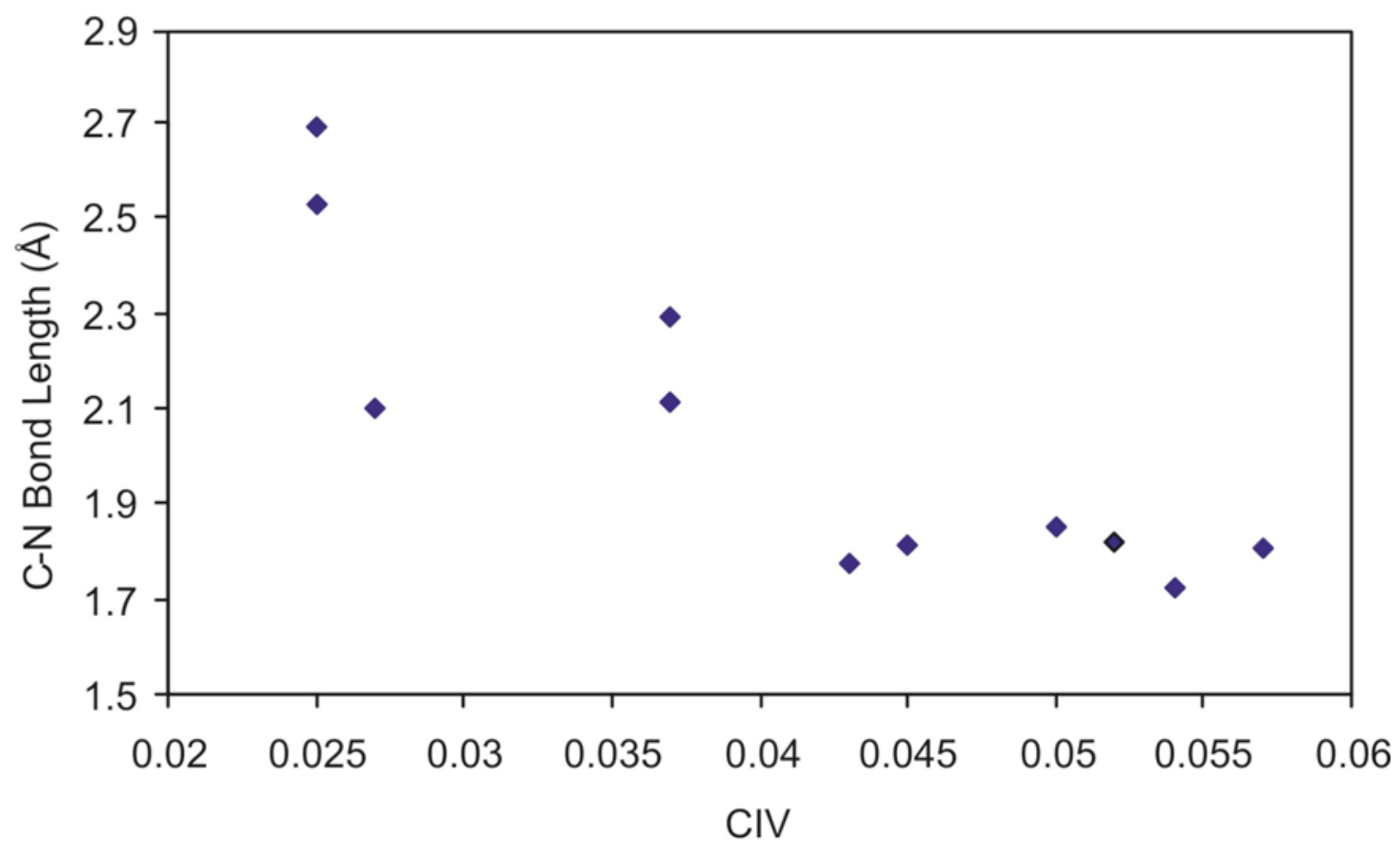

Figure S2. CIVs (critical isosurface values) of all transition states of the cyclization reactions plotted as a function of the bond length of the $\mathrm{C}-\mathrm{N}$ bond which is formed during the reaction. As expected, there is no linear relationship because the CIV does not represent the total electron density, but the density of delocalized electrons. Therefore, the CIV is a function of both the bond length as well as the "pseudo" character of the reaction. 

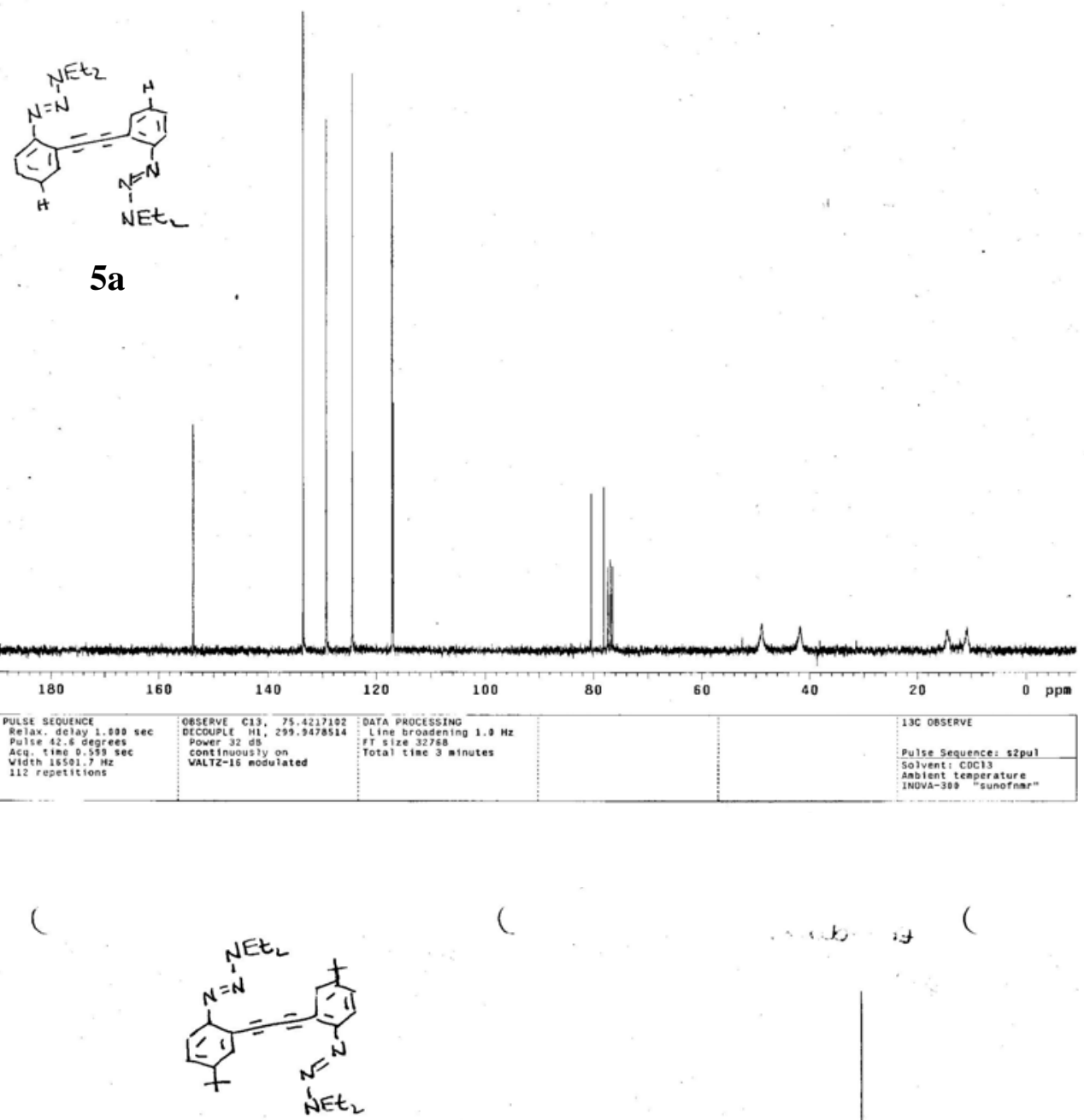

$5 b$

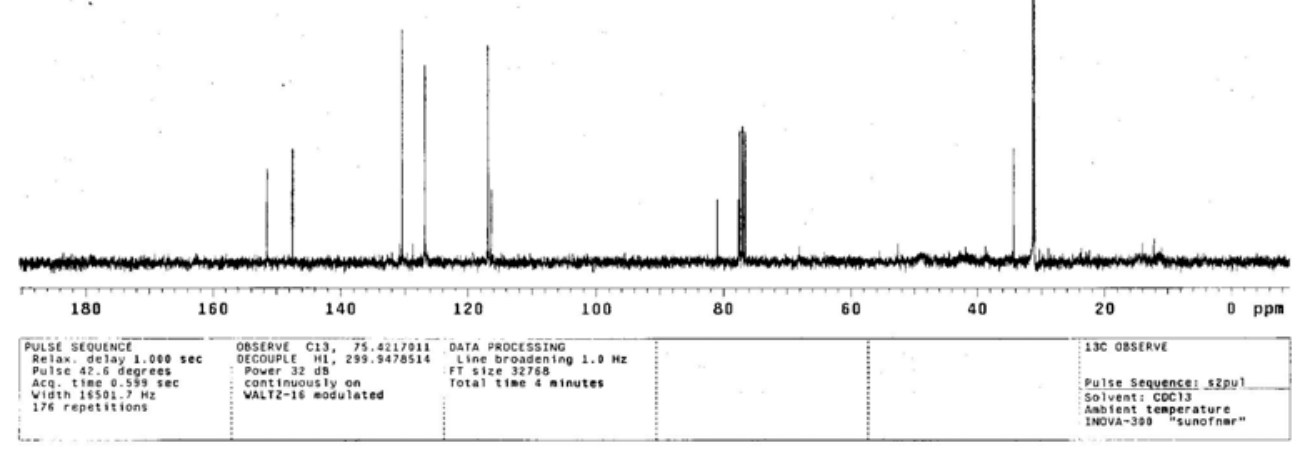

S10 

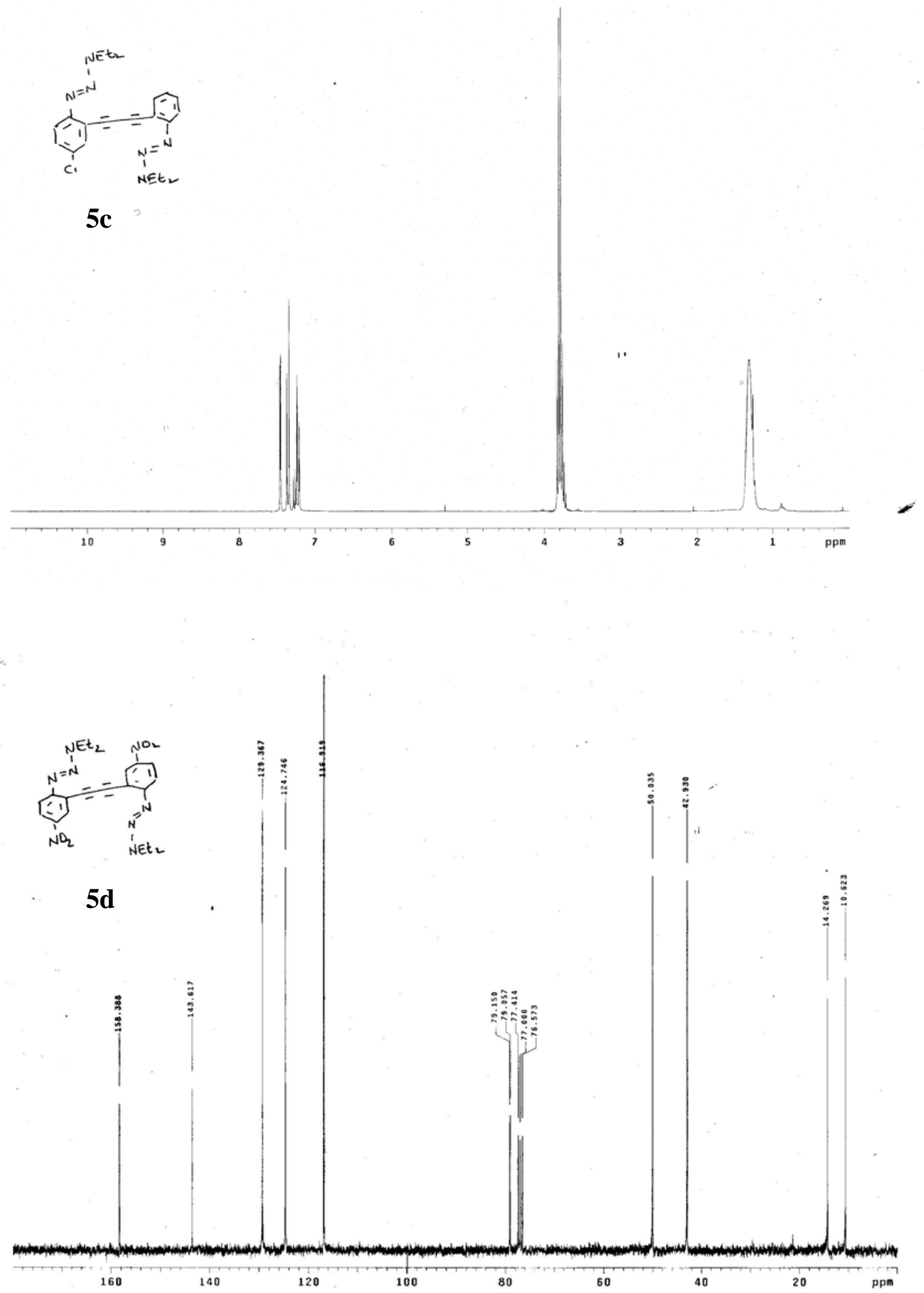

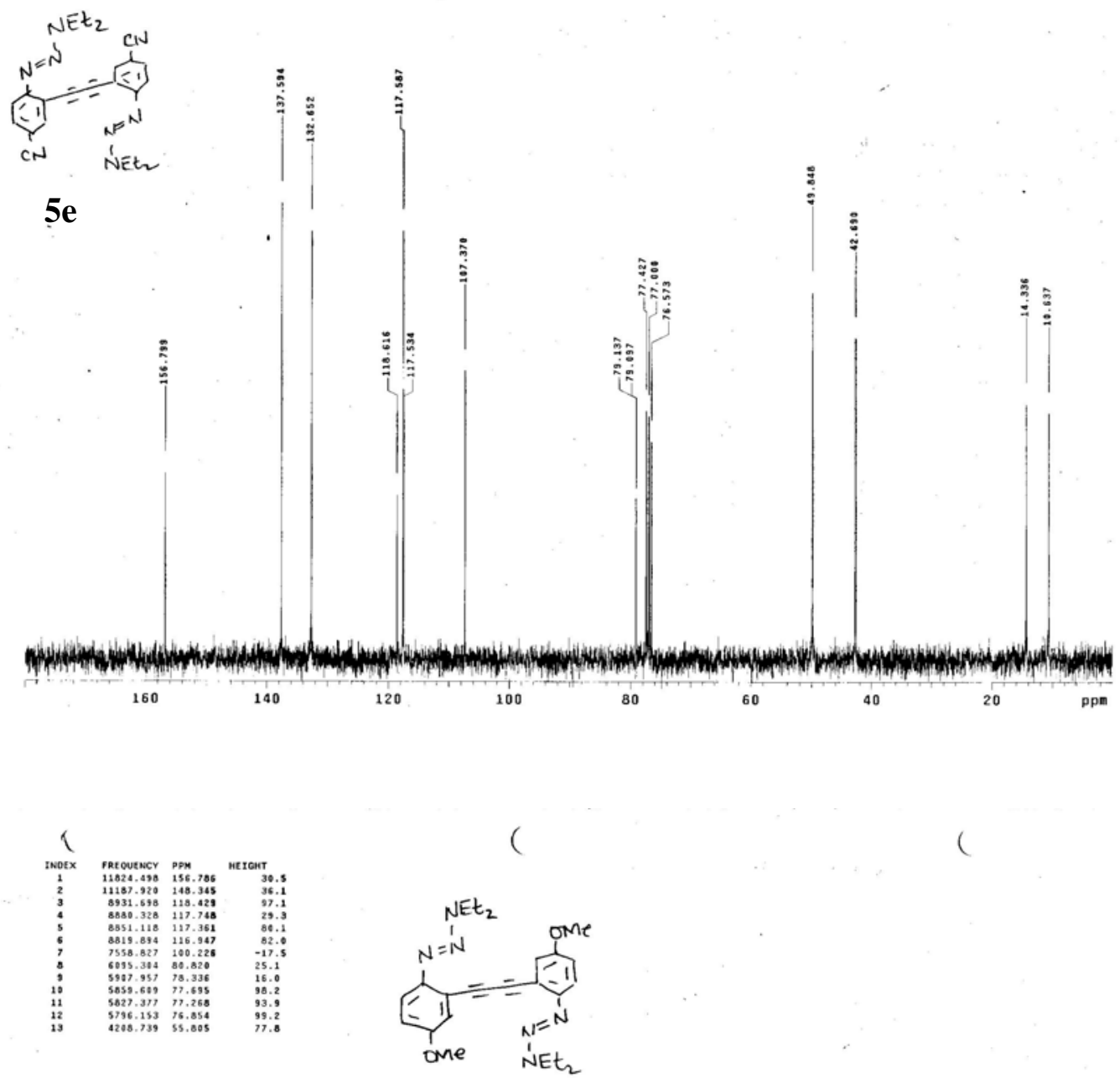

5f

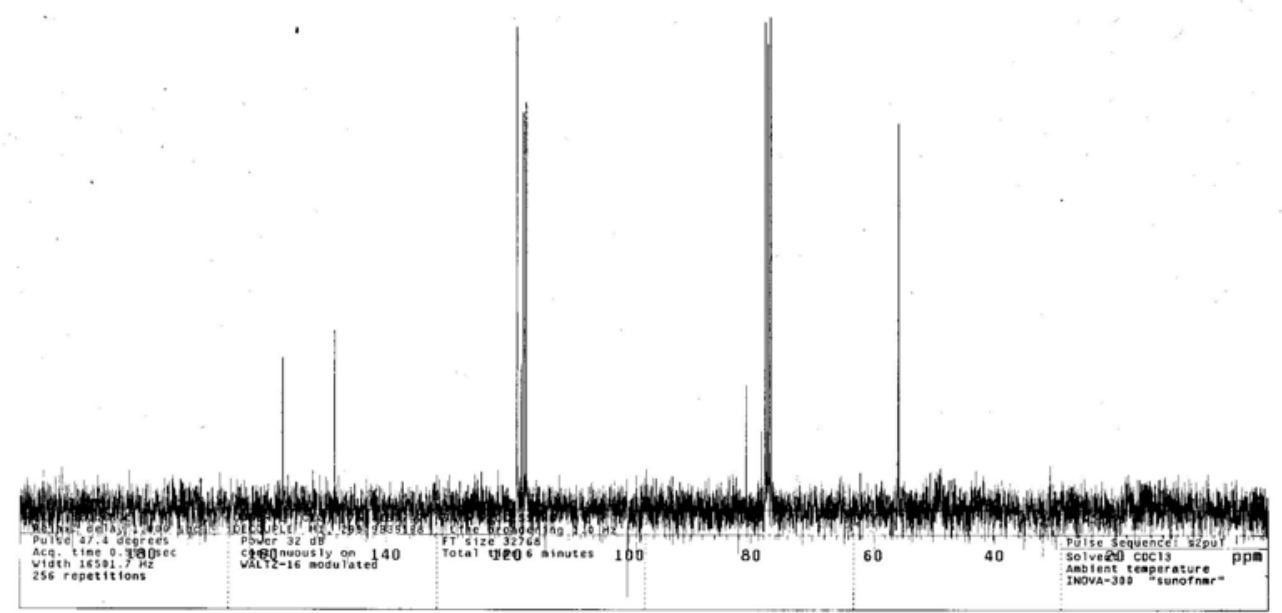



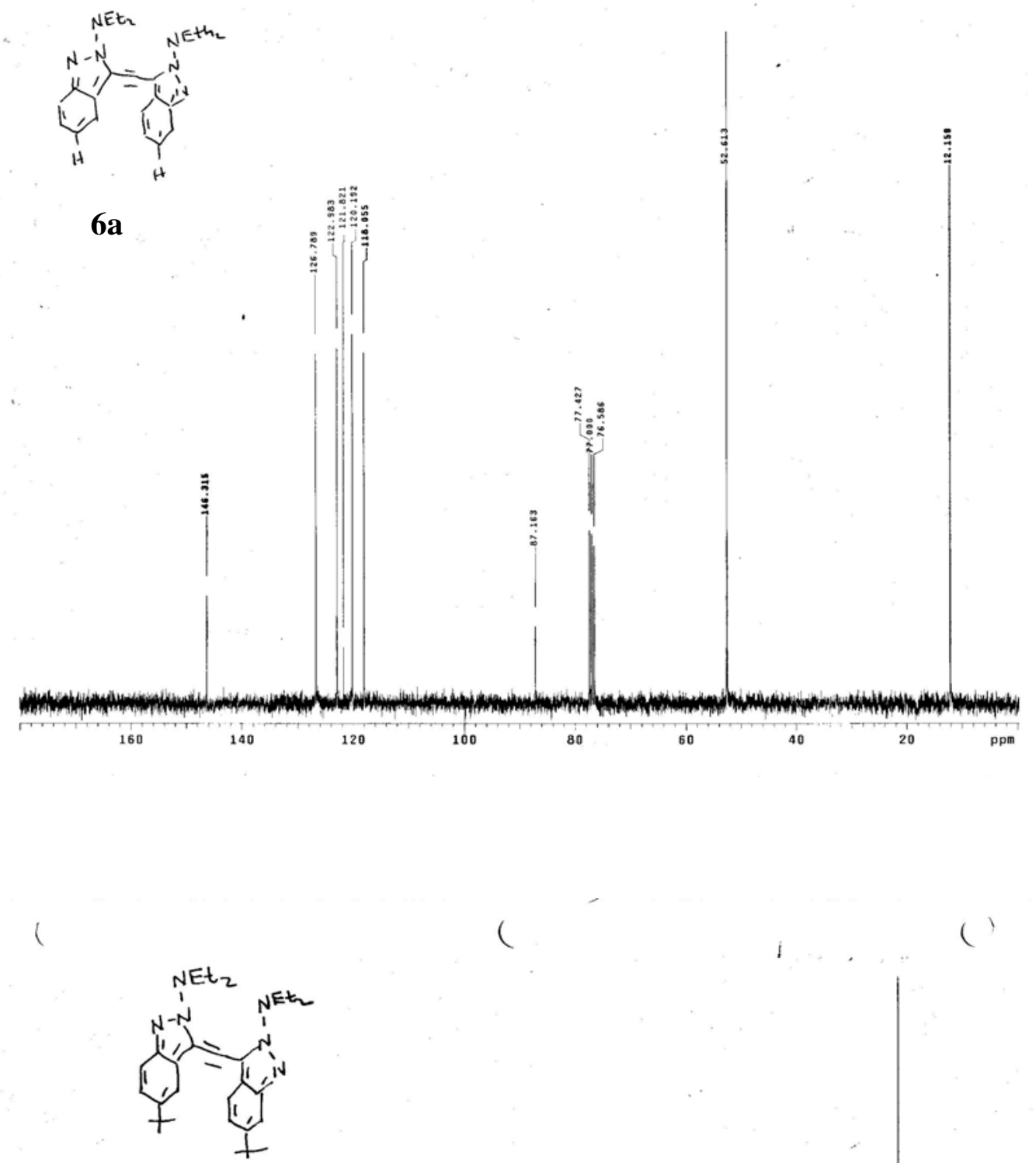

6b

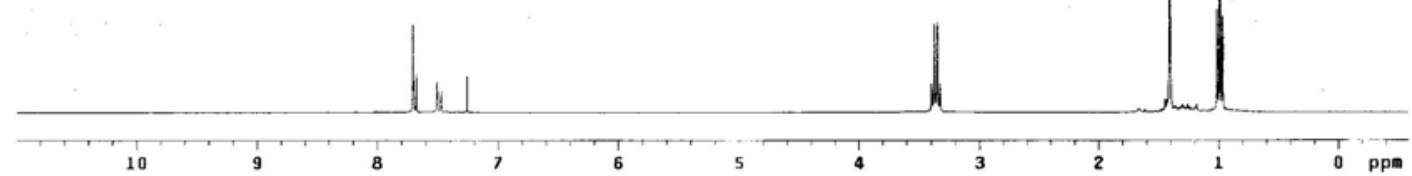




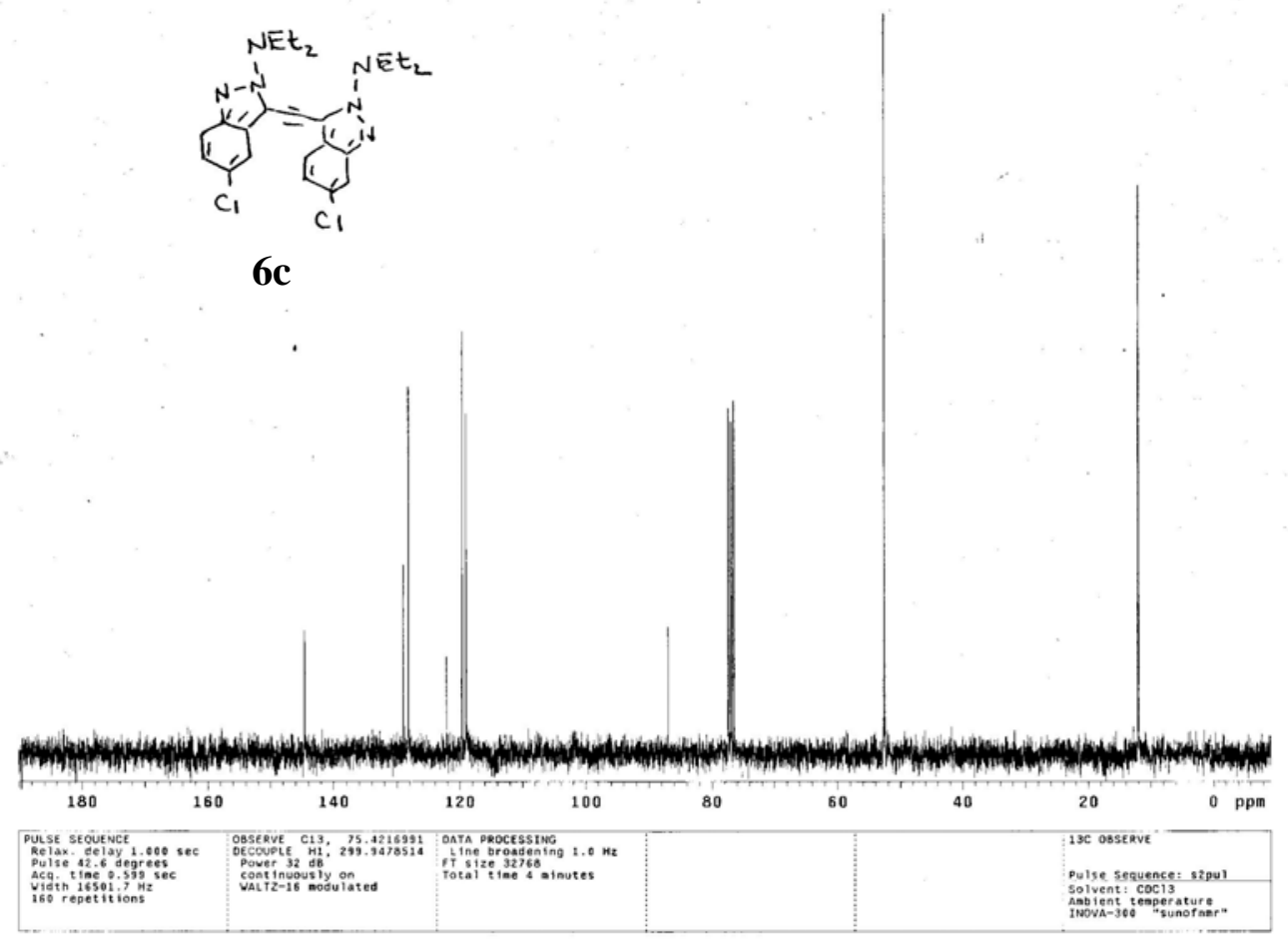

I

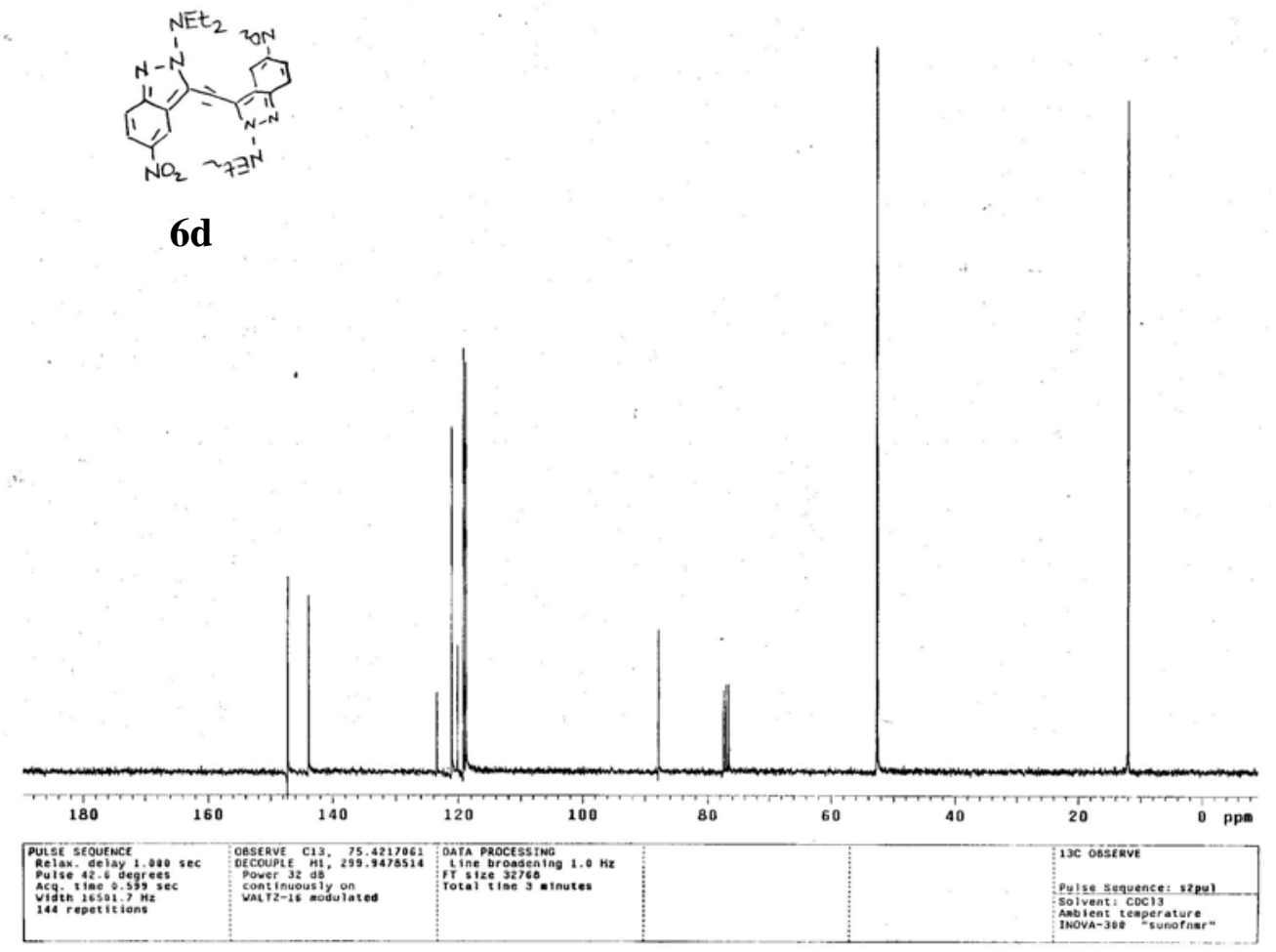



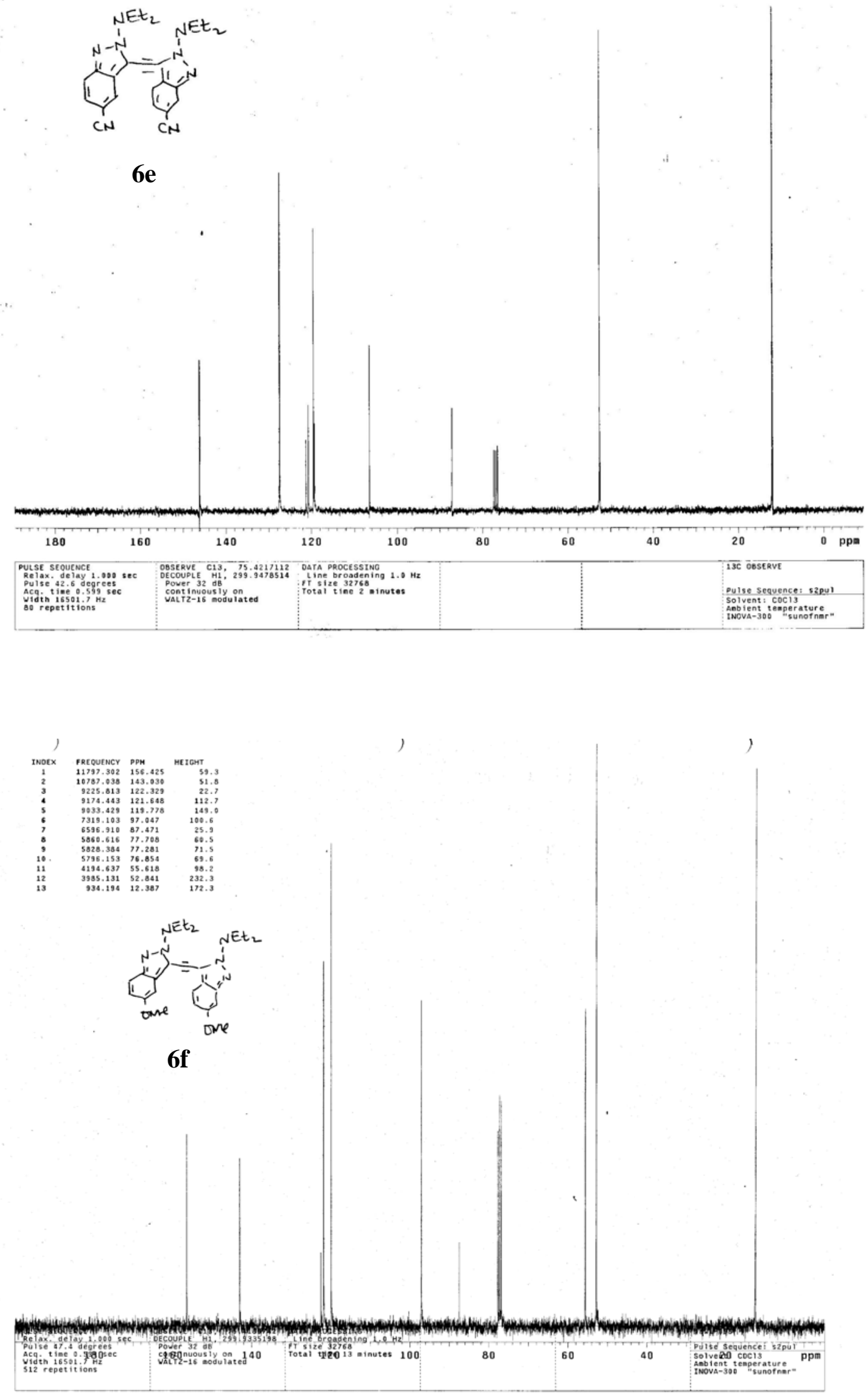

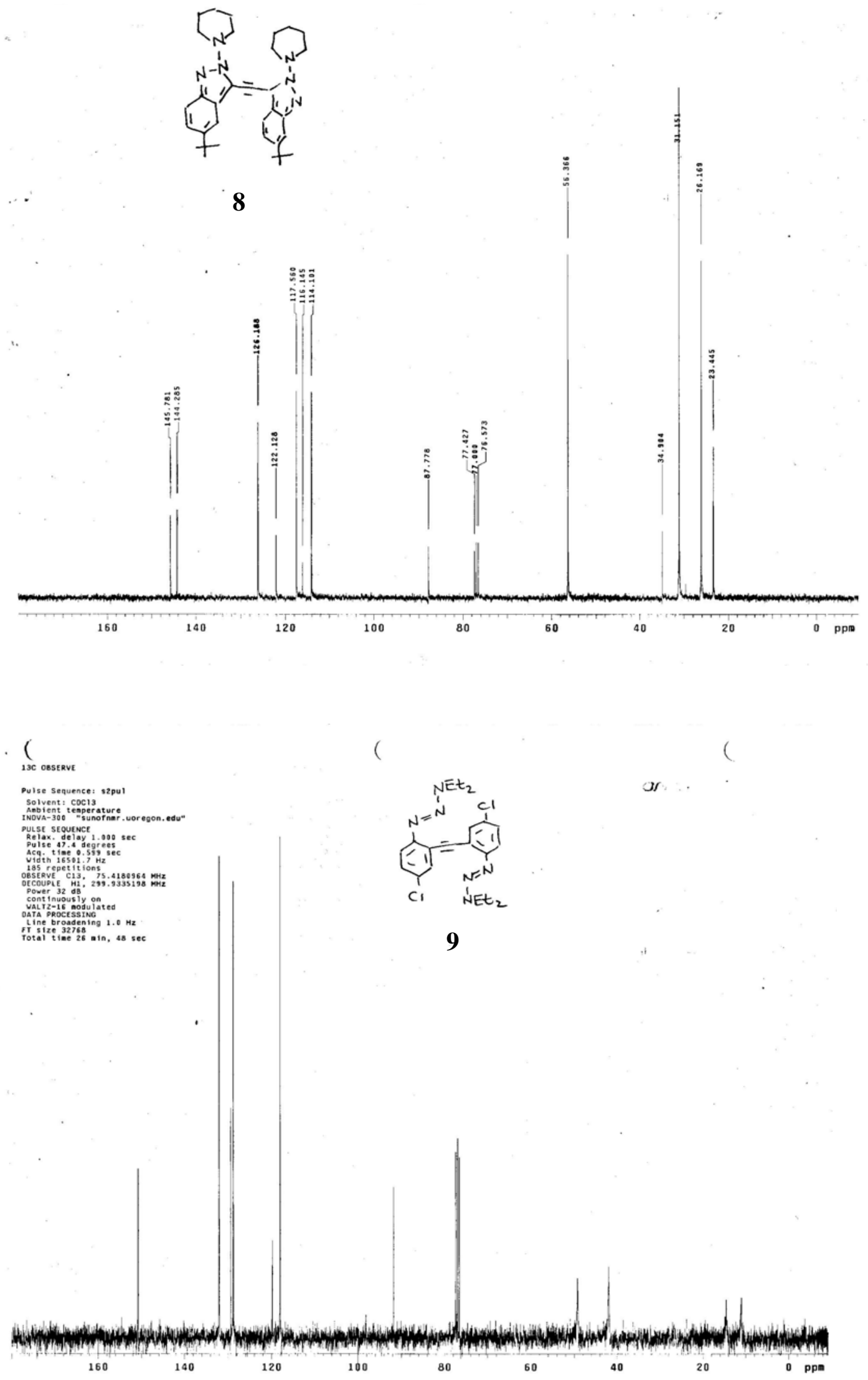


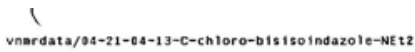

Pulse Sequence: szpul

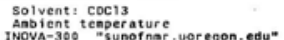

PULSE SEOUENCE
Relax. delay $1.000 \mathrm{sec}$

Pulse 47.4 degrees
Acg.

Videth $16501.7 \mathrm{~Hz}$

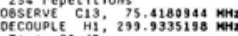

Power 32 dis
cont inuousty on
VALL T2-16 nodulated

line broasening $1.0 \mathrm{~Hz}$

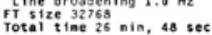

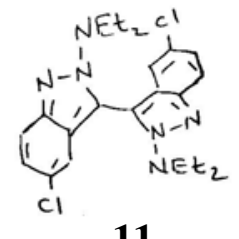

11

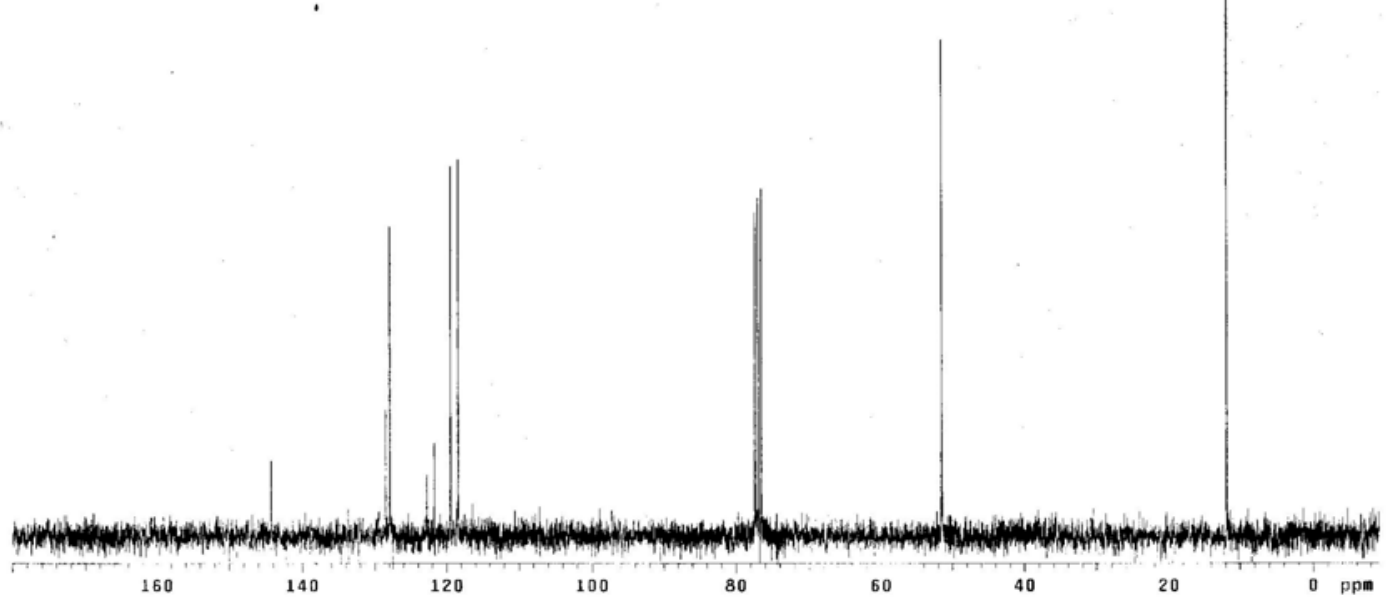

STANOARO IN OBSERVE

Pulse Sequence: s2pul

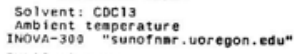

PULSE SEOUEnCE
Relax. Gelay $1.000 \mathrm{sec}$

ACO 37.9 degrees

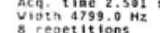

OESERVE H1 299.9325256 $\mathrm{MHz}$

DAFA brockssing $0.2 \mathrm{kz}$

tine broadening $0.2 \mathrm{kz}$
FT size $32768 \mathrm{~s}$
Iotal tine $0 \mathrm{sin}, 28 \mathrm{sec}$

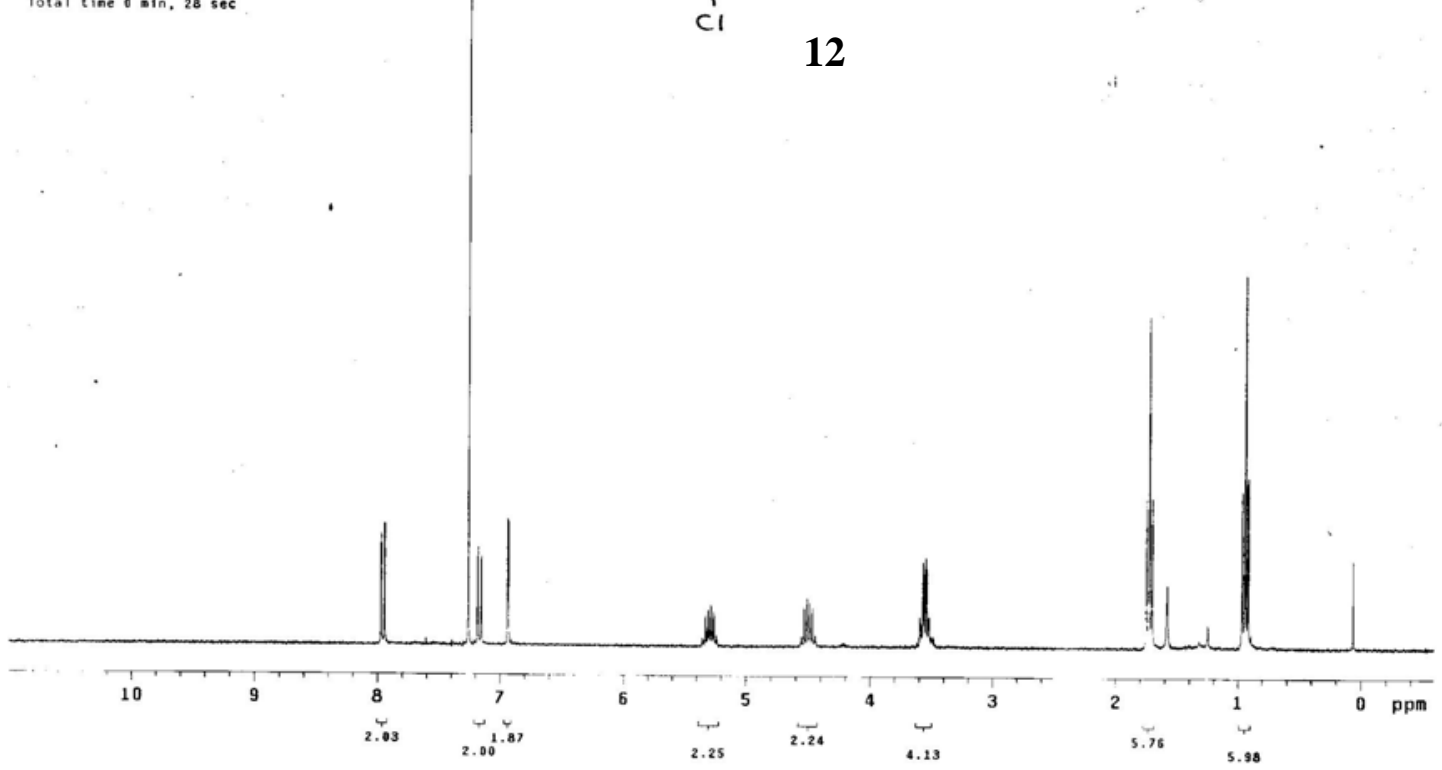

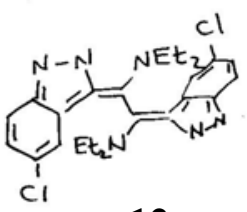

12 
X-ray Data for 12

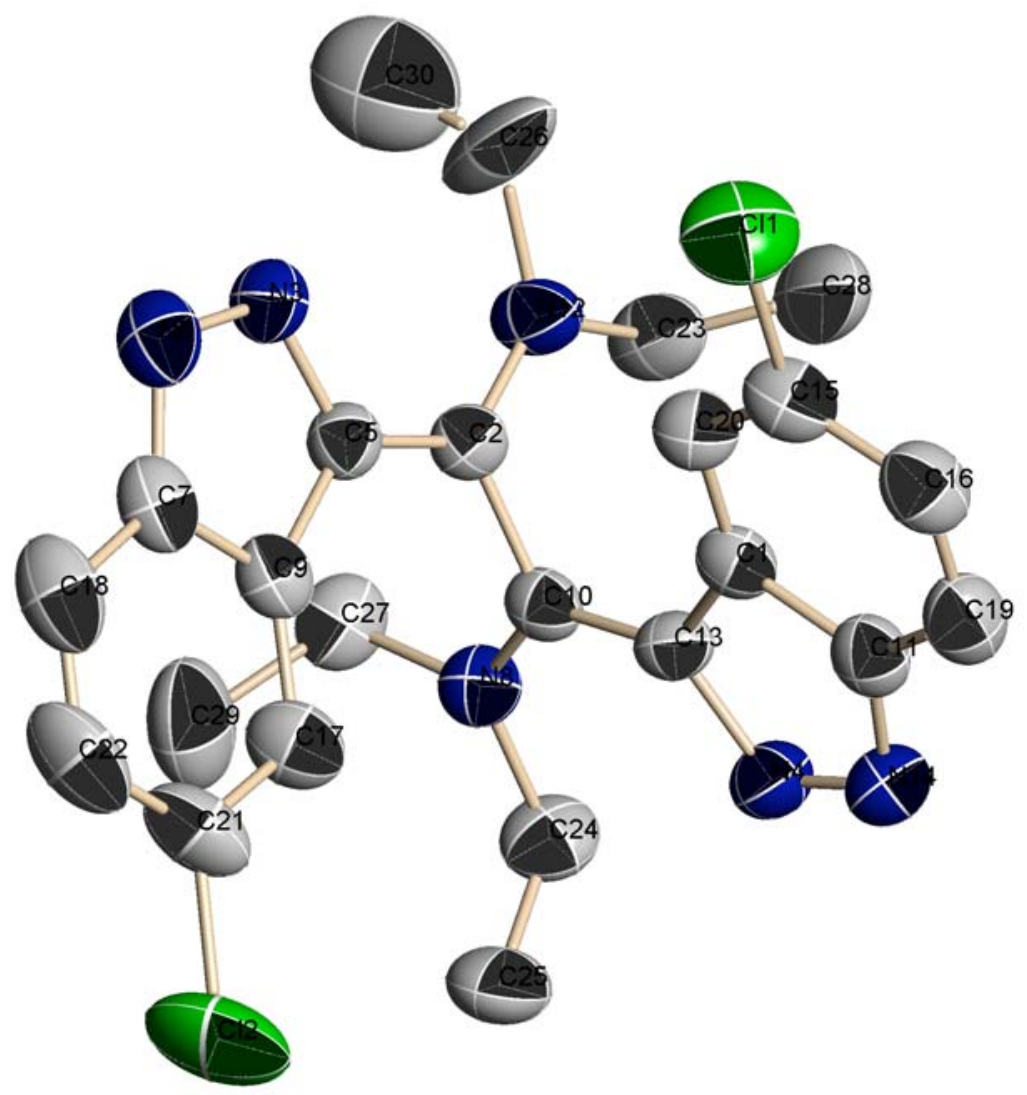

Molecular Structure of $\mathbf{1 2 .}$ 
Table 1. Crystal data and structure refinement for $\mathbf{1 2}$.

Empirical formula

Formula weight

Temperature

Wavelength

Crystal system

Space group

Unit cell dimensions

Volume

Z

Density (calculated)

Absorption coefficient

$\mathrm{F}(000)$

Crystal size

Theta range for data collection

Index ranges

Reflections collected

Independent reflections

Completeness to theta $=25.09^{\circ}$

Absorption correction

Max. and min. transmission

Refinement method

Data / restraints / parameters

Goodness-of-fit on $\mathrm{F}^{2}$

Final $\mathrm{R}$ indices [I $>2 \operatorname{sigma}(\mathrm{I})]$

$\mathrm{R}$ indices (all data)

Absolute structure parameter

Largest diff. peak and hole

\section{C24 H26 Cl2 N6}

469.41

153(2) K

$0.71073 \AA$

Monoclinic

P2(1)

$\mathrm{a}=8.3847(17) \AA$

$\alpha=90^{\circ}$.

$\mathrm{b}=15.410(3) \AA$

$\beta=113.862(3)^{\circ}$.

$c=9.877(2) \AA$

$\gamma=90^{\circ}$.

1167.1(4) $\AA^{3}$

2

\section{$1.336 \mathrm{Mg} / \mathrm{m}^{3}$}

$0.303 \mathrm{~mm}^{-1}$

492

$0.10 \times 0.10 \times 0.15 \mathrm{~mm}^{3}$

2.25 to $25.09^{\circ}$.

$-9<=\mathrm{h}<=9,-18<=\mathrm{k}<=18,-11<=\mathrm{l}<=11$

8452

$4083[\mathrm{R}(\mathrm{int})=0.0112]$

$99.4 \%$

Empirical

1.000 and 0.878

Full-matrix least-squares on $\mathrm{F}^{2}$

4083 / 15 / 293

1.047

$\mathrm{R} 1=0.0461, \mathrm{wR} 2=0.1244$

$\mathrm{R} 1=0.0476, \mathrm{wR} 2=0.1259$

$0.05(8)$

0.464 and -0.431 e. $\AA^{-3}$ 
Table 2. Atomic coordinates ( $\times 10^{4}$ ) and equivalent isotropic displacement parameters $\left(\AA^{2} \times 10^{3}\right)$ for 12. $U(e q)$ is defined as one third of the trace of the orthogonalized $U^{\mathrm{ij}}$ tensor.

\begin{tabular}{|c|c|c|c|c|}
\hline & $\mathrm{x}$ & $\mathrm{y}$ & $\mathrm{z}$ & $\mathrm{U}(\mathrm{eq})$ \\
\hline $\mathrm{Cl}(1)$ & $-591(2)$ & $5822(1)$ & $7705(2)$ & 111(1) \\
\hline $\mathrm{Cl}(2)$ & 207(1) & $3187(1)$ & $767(1)$ & $81(1)$ \\
\hline $\mathrm{N}(1)$ & $4665(4)$ & $3971(2)$ & 6344(3) & $57(1)$ \\
\hline $\mathrm{N}(2)$ & $4862(3)$ & $4489(2)$ & 5364(3) & $50(1)$ \\
\hline $\mathrm{N}(3)$ & $4500(3)$ & $5937(2)$ & $3239(3)$ & $57(1)$ \\
\hline $\mathrm{N}(4)$ & $-2025(3)$ & $6720(2)$ & $1276(3)$ & $57(1)$ \\
\hline $\mathrm{N}(5)$ & $-661(3)$ & $7033(2)$ & 2359(3) & $51(1)$ \\
\hline $\mathrm{N}(6)$ & 2901(3) & $7270(2)$ & $4805(3)$ & $47(1)$ \\
\hline $\mathrm{C}(1)$ & $983(5)$ & $5241(3)$ & $7328(4)$ & $69(1)$ \\
\hline$C(2)$ & $1620(6)$ & $4466(3)$ & 8086(4) & $78(1)$ \\
\hline$C(3)$ & $2844(5)$ & $3996(2)$ & 7808(4) & $68(1)$ \\
\hline$C(4)$ & $3453(4)$ & $4329(2)$ & 6790(3) & $52(1)$ \\
\hline$C(5)$ & 2813(4) & $5116(2)$ & 6042(3) & $45(1)$ \\
\hline$C(6)$ & 1544(4) & $5589(2)$ & 6311(4) & $58(1)$ \\
\hline$C(7)$ & $3770(3)$ & $5202(2)$ & $5122(3)$ & $42(1)$ \\
\hline $\mathrm{C}(8)$ & $3669(3)$ & $5870(2)$ & 4121(3) & $41(1)$ \\
\hline $\mathrm{C}(9)$ & 2350(3) & $6579(2)$ & $3938(3)$ & $41(1)$ \\
\hline$C(10)$ & $686(3)$ & $6419(2)$ & 2829(3) & $42(1)$ \\
\hline $\mathrm{C}(11)$ & 43(4) & $5643(2)$ & 1954(3) & $43(1)$ \\
\hline$C(12)$ & $627(4)$ & $4800(2)$ & 1883(3) & $48(1)$ \\
\hline$C(13)$ & $-508(4)$ & $4244(2)$ & $874(4)$ & $54(1)$ \\
\hline$C(14)$ & $-2193(5)$ & $4469(2)$ & $-102(4)$ & $62(1)$ \\
\hline$C(15)$ & $-2786(4)$ & $5295(2)$ & $-40(4)$ & $60(1)$ \\
\hline$C(16)$ & $-1662(4)$ & $5873(2)$ & $997(3)$ & $48(1)$ \\
\hline$C(17)$ & $4404(5)$ & $6728(2)$ & 2348(4) & $66(1)$ \\
\hline$C(18)$ & $3380(7)$ & $6591(3)$ & $741(5)$ & $92(1)$ \\
\hline$C(19)$ & $5525(7)$ & $5089(7)$ & $2875(9)$ & $181(5)$ \\
\hline$C(20)$ & $7058(17)$ & $5315(4)$ & $3867(11)$ & $220(7)$ \\
\hline$C(21)$ & $4740(4)$ & $7346(2)$ & 5923(4) & $59(1)$ \\
\hline$C(22)$ & $4865(6)$ & $7341(4)$ & 7483(4) & $96(2)$ \\
\hline $\mathrm{C}(23)$ & 1815(5) & $8054(2)$ & 4652(4) & $60(1)$ \\
\hline$C(24)$ & $586(5)$ & 7990(3) & $5423(5)$ & $77(1)$ \\
\hline
\end{tabular}


Table 3. Bond lengths [A] for $\mathbf{1 2}$.

\begin{tabular}{|c|c|}
\hline $\mathrm{Cl}(1)-\mathrm{C}(1)$ & $1.755(4)$ \\
\hline $\mathrm{Cl}(2)-\mathrm{C}(13)$ & $1.754(3)$ \\
\hline $\mathrm{N}(1)-\mathrm{N}(2)$ & $1.315(4)$ \\
\hline $\mathrm{N}(1)-\mathrm{C}(4)$ & $1.375(5)$ \\
\hline $\mathrm{N}(2)-\mathrm{C}(7)$ & $1.388(4)$ \\
\hline $\mathrm{N}(3)-\mathrm{C}(8)$ & $1.321(3)$ \\
\hline $\mathrm{N}(3)-\mathrm{C}(17)$ & $1.487(4)$ \\
\hline $\mathrm{N}(3)-\mathrm{C}(19)$ & $1.681(10)$ \\
\hline N(4)-N(5) & $1.301(4)$ \\
\hline $\mathrm{N}(4)-\mathrm{C}(16)$ & $1.393(4)$ \\
\hline $\mathrm{N}(5)-\mathrm{C}(10)$ & $1.400(4)$ \\
\hline $\mathrm{N}(6)-\mathrm{C}(9)$ & $1.326(4)$ \\
\hline $\mathrm{N}(6)-\mathrm{C}(23)$ & $1.484(4)$ \\
\hline $\mathrm{N}(6)-\mathrm{C}(21)$ & $1.494(4)$ \\
\hline $\mathrm{C}(1)-\mathrm{C}(6)$ & $1.378(5)$ \\
\hline $\mathrm{C}(1)-\mathrm{C}(2)$ & $1.396(6)$ \\
\hline$C(2)-C(3)$ & $1.372(6)$ \\
\hline$C(3)-C(4)$ & $1.397(5)$ \\
\hline$C(4)-C(5)$ & $1.408(4)$ \\
\hline$C(5)-C(6)$ & $1.402(4)$ \\
\hline$C(5)-C(7)$ & $1.441(4)$ \\
\hline$C(7)-C(8)$ & $1.407(4)$ \\
\hline $\mathrm{C}(8)-\mathrm{C}(9)$ & $1.512(4)$ \\
\hline C(9)-C(10) & $1.404(4)$ \\
\hline $\mathrm{C}(10)-\mathrm{C}(11)$ & $1.446(4)$ \\
\hline $\mathrm{C}(11)-\mathrm{C}(12)$ & $1.399(4)$ \\
\hline $\mathrm{C}(11)-\mathrm{C}(16)$ & $1.404(4)$ \\
\hline $\mathrm{C}(12)-\mathrm{C}(13)$ & $1.366(4)$ \\
\hline $\mathrm{C}(13)-\mathrm{C}(14)$ & $1.395(5)$ \\
\hline$C(14)-C(15)$ & $1.377(5)$ \\
\hline$C(15)-C(16)$ & $1.397(4)$ \\
\hline $\mathrm{C}(17)-\mathrm{C}(18)$ & $1.483(6)$ \\
\hline$C(19)-C(20)$ & $1.309(12)$ \\
\hline $\mathrm{C}(21)-\mathrm{C}(22)$ & $1.501(6)$ \\
\hline$C(23)-C(24)$ & $1.512(5)$ \\
\hline
\end{tabular}


Table 4. Bond angles $\left[{ }^{\circ}\right]$ for $\mathbf{1 2}$.

\begin{tabular}{|c|c|}
\hline $\mathrm{N}(2)-\mathrm{N}(1)-\mathrm{C}(4)$ & $108.7(2)$ \\
\hline $\mathrm{N}(1)-\mathrm{N}(2)-\mathrm{C}(7)$ & $109.7(2)$ \\
\hline $\mathrm{C}(8)-\mathrm{N}(3)-\mathrm{C}(17)$ & $122.5(3)$ \\
\hline $\mathrm{C}(8)-\mathrm{N}(3)-\mathrm{C}(19)$ & $122.1(3)$ \\
\hline C(17)-N(3)-C(19) & $114.9(3)$ \\
\hline $\mathrm{N}(5)-\mathrm{N}(4)-\mathrm{C}(16)$ & $108.7(2)$ \\
\hline $\mathrm{N}(4)-\mathrm{N}(5)-\mathrm{C}(10)$ & $110.2(3)$ \\
\hline $\mathrm{C}(9)-\mathrm{N}(6)-\mathrm{C}(23)$ & $123.3(2)$ \\
\hline $\mathrm{C}(9)-\mathrm{N}(6)-\mathrm{C}(21)$ & $121.8(3)$ \\
\hline $\mathrm{C}(23)-\mathrm{N}(6)-\mathrm{C}(21)$ & $114.7(2)$ \\
\hline $\mathrm{C}(6)-\mathrm{C}(1)-\mathrm{C}(2)$ & $123.7(4)$ \\
\hline $\mathrm{C}(6)-\mathrm{C}(1)-\mathrm{Cl}(1)$ & $117.6(3)$ \\
\hline $\mathrm{C}(2)-\mathrm{C}(1)-\mathrm{Cl}(1)$ & $118.7(3)$ \\
\hline $\mathrm{C}(3)-\mathrm{C}(2)-\mathrm{C}(1)$ & $119.9(3)$ \\
\hline$C(2)-C(3)-C(4)$ & 118.1(3) \\
\hline $\mathrm{N}(1)-\mathrm{C}(4)-\mathrm{C}(3)$ & $128.0(3)$ \\
\hline $\mathrm{N}(1)-\mathrm{C}(4)-\mathrm{C}(5)$ & $110.6(3)$ \\
\hline$C(3)-C(4)-C(5)$ & $121.4(3)$ \\
\hline $\mathrm{C}(6)-\mathrm{C}(5)-\mathrm{C}(4)$ & $120.4(3)$ \\
\hline$C(6)-C(5)-C(7)$ & $137.0(3)$ \\
\hline$C(4)-C(5)-C(7)$ & $102.6(3)$ \\
\hline$C(1)-C(6)-C(5)$ & $116.4(3)$ \\
\hline $\mathrm{N}(2)-\mathrm{C}(7)-\mathrm{C}(8)$ & $123.3(2)$ \\
\hline $\mathrm{N}(2)-\mathrm{C}(7)-\mathrm{C}(5)$ & $108.4(2)$ \\
\hline $\mathrm{C}(8)-\mathrm{C}(7)-\mathrm{C}(5)$ & $128.3(3)$ \\
\hline $\mathrm{N}(3)-\mathrm{C}(8)-\mathrm{C}(7)$ & $128.5(3)$ \\
\hline $\mathrm{N}(3)-\mathrm{C}(8)-\mathrm{C}(9)$ & $114.9(2)$ \\
\hline $\mathrm{C}(7)-\mathrm{C}(8)-\mathrm{C}(9)$ & $116.5(2)$ \\
\hline $\mathrm{N}(6)-\mathrm{C}(9)-\mathrm{C}(10)$ & $128.4(3)$ \\
\hline N(6)-C(9)-C(8) & $117.1(2)$ \\
\hline $\mathrm{C}(10)-\mathrm{C}(9)-\mathrm{C}(8)$ & $114.5(2)$ \\
\hline $\mathrm{C}(9)-\mathrm{C}(10)-\mathrm{N}(5)$ & $123.6(3)$ \\
\hline $\mathrm{C}(9)-\mathrm{C}(10)-\mathrm{C}(11)$ & $128.6(2)$ \\
\hline $\mathrm{N}(5)-\mathrm{C}(10)-\mathrm{C}(11)$ & $107.8(2)$ \\
\hline $\mathrm{C}(12)-\mathrm{C}(11)-\mathrm{C}(16)$ & $118.8(3)$ \\
\hline $\mathrm{C}(12)-\mathrm{C}(11)-\mathrm{C}(10)$ & 138.1(3) \\
\hline $\mathrm{C}(16)-\mathrm{C}(11)-\mathrm{C}(10)$ & $103.0(2)$ \\
\hline
\end{tabular}




$\begin{array}{lr}\mathrm{C}(13)-\mathrm{C}(12)-\mathrm{C}(11) & 117.7(3) \\ \mathrm{C}(12)-\mathrm{C}(13)-\mathrm{C}(14) & 124.1(3) \\ \mathrm{C}(12)-\mathrm{C}(13)-\mathrm{Cl}(2) & 118.2(3) \\ \mathrm{C}(14)-\mathrm{C}(13)-\mathrm{Cl}(2) & 117.7(2) \\ \mathrm{C}(15)-\mathrm{C}(14)-\mathrm{C}(13) & 118.8(3) \\ \mathrm{C}(14)-\mathrm{C}(15)-\mathrm{C}(16) & 118.3(3) \\ \mathrm{N}(4)-\mathrm{C}(16)-\mathrm{C}(15) & 127.3(3) \\ \mathrm{N}(4)-\mathrm{C}(16)-\mathrm{C}(11) & 110.4(2) \\ \mathrm{C}(15)-\mathrm{C}(16)-\mathrm{C}(11) & 122.3(3) \\ \mathrm{C}(18)-\mathrm{C}(17)-\mathrm{N}(3) & 112.7(4) \\ \mathrm{C}(20)-\mathrm{C}(19)-\mathrm{N}(3) & 93.7(9) \\ \mathrm{N}(6)-\mathrm{C}(21)-\mathrm{C}(22) & 112.4(3) \\ \mathrm{N}(6)-\mathrm{C}(23)-\mathrm{C}(24) & 114.3(3)\end{array}$

Symmetry transformations used to generate equivalent atoms: 
Table 5. Anisotropic displacement parameters $\left(\AA^{2} \times 10^{3}\right)$ for 12. The anisotropic displacement factor exponent takes the form: $-2 \pi^{2}\left[h^{2} a^{* 2} U^{11}+\ldots+2 h k a^{*} b^{*} U^{12}\right]$

\begin{tabular}{|c|c|c|c|c|c|c|}
\hline & $\mathrm{U}^{11}$ & $\mathrm{U}^{22}$ & $\mathrm{U}^{33}$ & $\mathrm{U}^{23}$ & $\mathrm{U}^{13}$ & $\mathrm{U}^{12}$ \\
\hline $\mathrm{Cl}(1)$ & $130(1)$ & $126(1)$ & $129(1)$ & $-8(1)$ & $106(1)$ & $-3(1)$ \\
\hline $\mathrm{Cl}(2)$ & $93(1)$ & $49(1)$ & $100(1)$ & $-18(1)$ & $39(1)$ & $-10(1)$ \\
\hline $\mathrm{N}(1)$ & 71(2) & $44(1)$ & $55(1)$ & $9(1)$ & $24(1)$ & 1(1) \\
\hline $\mathrm{N}(2)$ & $58(1)$ & $43(1)$ & $49(1)$ & $8(1)$ & $23(1)$ & $6(1)$ \\
\hline $\mathrm{N}(3)$ & $63(2)$ & $56(2)$ & $68(2)$ & 21(1) & $43(1)$ & $14(1)$ \\
\hline $\mathrm{N}(4)$ & $48(1)$ & $64(2)$ & $58(2)$ & $4(1)$ & $21(1)$ & $7(1)$ \\
\hline $\mathrm{N}(5)$ & $47(1)$ & $53(1)$ & $54(1)$ & $4(1)$ & $22(1)$ & $10(1)$ \\
\hline $\mathrm{N}(6)$ & $49(1)$ & $41(1)$ & $49(1)$ & $0(1)$ & $19(1)$ & $-3(1)$ \\
\hline $\mathrm{C}(1)$ & $85(2)$ & $74(2)$ & $68(2)$ & $-9(2)$ & $50(2)$ & $-19(2)$ \\
\hline$C(2)$ & $106(3)$ & $84(3)$ & $63(2)$ & $-2(2)$ & $55(2)$ & $-31(2)$ \\
\hline$C(3)$ & $95(3)$ & $55(2)$ & $54(2)$ & $5(2)$ & $31(2)$ & $-21(2)$ \\
\hline $\mathrm{C}(4)$ & $62(2)$ & $47(2)$ & $44(2)$ & $0(1)$ & $18(1)$ & $-17(1)$ \\
\hline$C(5)$ & $50(2)$ & $43(1)$ & $41(1)$ & $-2(1)$ & $19(1)$ & $-10(1)$ \\
\hline$C(6)$ & $62(2)$ & $59(2)$ & $65(2)$ & $0(2)$ & $38(2)$ & $-6(2)$ \\
\hline$C(7)$ & $43(1)$ & $41(1)$ & $43(1)$ & $-1(1)$ & 19(1) & $-1(1)$ \\
\hline $\mathrm{C}(8)$ & $39(1)$ & $40(1)$ & $45(1)$ & $3(1)$ & $17(1)$ & $-3(1)$ \\
\hline $\mathrm{C}(9)$ & $44(1)$ & $40(1)$ & $44(1)$ & $6(1)$ & $25(1)$ & $0(1)$ \\
\hline $\mathrm{C}(10)$ & $42(1)$ & $42(1)$ & $48(1)$ & 2(1) & $24(1)$ & 2(1) \\
\hline $\mathrm{C}(11)$ & $47(1)$ & $46(2)$ & $45(1)$ & $1(1)$ & $27(1)$ & $-4(1)$ \\
\hline$C(12)$ & $50(2)$ & $46(2)$ & $52(2)$ & $-1(1)$ & $24(1)$ & $-4(1)$ \\
\hline $\mathrm{C}(13)$ & $57(2)$ & $51(2)$ & $61(2)$ & $-6(1)$ & $31(2)$ & $-11(1)$ \\
\hline$C(14)$ & $60(2)$ & 71(2) & $56(2)$ & $-14(2)$ & $25(2)$ & $-18(2)$ \\
\hline $\mathrm{C}(15)$ & $49(2)$ & $81(2)$ & $49(2)$ & $-1(2)$ & $18(1)$ & $-5(2)$ \\
\hline$C(16)$ & $46(1)$ & $57(2)$ & $44(1)$ & 1(1) & $22(1)$ & $-5(1)$ \\
\hline $\mathrm{C}(17)$ & $70(2)$ & $65(2)$ & $84(2)$ & $30(2)$ & $52(2)$ & $13(2)$ \\
\hline$C(18)$ & $113(3)$ & $100(3)$ & $79(3)$ & $45(2)$ & $57(2)$ & $48(3)$ \\
\hline$C(19)$ & $70(3)$ & $307(11)$ & $202(7)$ & $203(8)$ & $92(4)$ & $100(5)$ \\
\hline $\mathrm{C}(20)$ & $490(20)$ & 71(4) & $237(11)$ & $3(5)$ & $286(14)$ & $-11(7)$ \\
\hline$C(21)$ & $53(2)$ & $50(2)$ & $70(2)$ & $-1(2)$ & $21(2)$ & $-9(1)$ \\
\hline $\mathrm{C}(22)$ & $79(3)$ & $128(4)$ & $59(2)$ & $4(2)$ & $6(2)$ & $-24(3)$ \\
\hline$C(23)$ & $65(2)$ & $42(2)$ & 71(2) & $-7(2)$ & $26(2)$ & 1(1) \\
\hline $\mathrm{C}(24)$ & $79(2)$ & $84(3)$ & $74(2)$ & $-14(2)$ & $36(2)$ & $18(2)$ \\
\hline
\end{tabular}


Table 6. Hydrogen coordinates ( x $10^{4}$ ) and isotropic displacement parameters $\left(\AA^{2} \mathrm{x} 10^{3}\right.$ ) for $\mathbf{1 2}$.

\begin{tabular}{|c|c|c|c|c|}
\hline & $\mathrm{x}$ & $\mathrm{y}$ & $\mathrm{z}$ & $\mathrm{U}(\mathrm{eq})$ \\
\hline $\mathrm{H}(2)$ & 1206 & 4264 & 8794 & 93 \\
\hline $\mathrm{H}(3)$ & 3265 & 3459 & 8294 & 81 \\
\hline $\mathrm{H}(6)$ & 1094 & 6121 & 5819 & 70 \\
\hline $\mathrm{H}(12)$ & 1772 & 4621 & 2514 & 58 \\
\hline $\mathrm{H}(14)$ & -2920 & 4060 & -798 & 74 \\
\hline $\mathrm{H}(15)$ & -3929 & 5467 & -687 & 72 \\
\hline $\mathrm{H}(17 \mathrm{~A})$ & 3866 & 7203 & 2692 & 80 \\
\hline $\mathrm{H}(17 \mathrm{~B})$ & 5601 & 6911 & 2513 & 80 \\
\hline $\mathrm{H}(18 \mathrm{~A})$ & 3889 & 6111 & 398 & 137 \\
\hline $\mathrm{H}(18 \mathrm{~B})$ & 3402 & 7121 & 201 & 137 \\
\hline $\mathrm{H}(18 \mathrm{C})$ & 2172 & 6449 & 562 & 137 \\
\hline $\mathrm{H}(19 \mathrm{~A})$ & 5122 & 4522 & 3093 & 217 \\
\hline $\mathrm{H}(19 \mathrm{~B})$ & 5465 & 5095 & 1853 & 217 \\
\hline $\mathrm{H}(20 \mathrm{~A})$ & 7300 & 5918 & 3698 & 331 \\
\hline $\mathrm{H}(20 \mathrm{~B})$ & 7954 & 4935 & 3787 & 331 \\
\hline $\mathrm{H}(20 \mathrm{C})$ & 7067 & 5260 & 4859 & 331 \\
\hline $\mathrm{H}(21 \mathrm{~A})$ & 5250 & 7892 & 5749 & 71 \\
\hline $\mathrm{H}(21 \mathrm{~B})$ & 5432 & 6858 & 5793 & 71 \\
\hline $\mathrm{H}(22 \mathrm{~A})$ & 4255 & 7848 & 7640 & 143 \\
\hline $\mathrm{H}(22 \mathrm{~B})$ & 6094 & 7361 & 8176 & 143 \\
\hline $\mathrm{H}(22 \mathrm{C})$ & 4328 & 6810 & 7652 & 143 \\
\hline $\mathrm{H}(23 \mathrm{~A})$ & 1122 & 8164 & 3586 & 72 \\
\hline $\mathrm{H}(23 \mathrm{~B})$ & 2595 & 8558 & 5057 & 72 \\
\hline $\mathrm{H}(24 \mathrm{~A})$ & -291 & 7542 & 4943 & 116 \\
\hline $\mathrm{H}(24 \mathrm{~B})$ & 6 & 8550 & 5360 & 116 \\
\hline $\mathrm{H}(24 \mathrm{C})$ & 1247 & 7837 & 6465 & 116 \\
\hline
\end{tabular}




\section{Cartesian coordinated for calculated structures}

\section{syn-14}

Point Group: C1

B3LYP/6-31G* $=-1296.74793 \mathrm{au}$

B3LYP/6-31G* Zero Point Corrected Energy $=-1296.35945$ au $\mathrm{NIMAG}=0$

B3LYP/6-31G* Optimized Cartesian Coordinates

$\begin{array}{lrrr}\mathrm{C} & 0.58260400 & -0.69667200 & -0.08299100 \\ \mathrm{C} & 1.97596300 & -0.84504100 & -0.00238100 \\ \mathrm{C} & 2.73027700 & -1.92867900 & 0.49332900 \\ \mathrm{C} & 2.41339100 & -3.20562000 & 1.00891300 \\ \mathrm{C} & 3.45471800 & -4.01080100 & 1.41530500 \\ \mathrm{C} & 4.08926700 & -1.49109200 & 0.41008700 \\ \mathrm{C} & 4.81215700 & -3.57831000 & 1.32448000 \\ \mathrm{C} & 5.14574000 & -2.33780600 & 0.83151300 \\ \mathrm{~N} & 4.18089700 & -0.24470900 & -0.09644400 \\ \mathrm{~N} & 2.90616900 & 0.11796700 & -0.34646800 \\ \mathrm{C} & 2.66205100 & 1.41318400 & -0.89149900 \\ \mathrm{H} & 1.38077800 & -3.53455200 & 1.08028600 \\ \mathrm{H} & 3.24599900 & -4.99904900 & 1.81551700 \\ \mathrm{H} & 5.59731300 & -4.25215300 & 1.65654400 \\ \mathrm{H} & 6.17574600 & -2.00286900 & 0.76074800 \\ \mathrm{C} & -0.63617000 & -0.62879200 & -0.08950200 \\ \mathrm{C} & -2.03853600 & -0.64702200 & -0.14051500 \\ \mathrm{C} & -2.89085800 & -1.69332200 & -0.55195800 \\ \mathrm{C} & -2.69036200 & -2.98559700 & -1.08719400 \\ \mathrm{C} & -3.80522200 & -3.74482600 & -1.36861300 \\ \mathrm{C} & -4.21055400 & -1.20000400 & -0.30997000 \\ \mathrm{C} & -5.12331700 & -3.25135500 & -1.13128200 \\ \mathrm{C} & -5.34403200 & -1.99695600 & -0.61018200 \\ \mathrm{~N} & -4.18868300 & 0.04773800 & 0.20021500 \\ \mathrm{~N} & -2.88053100 & 0.36138100 & 0.29210300 \\ \mathrm{C} & -2.52411700 & 1.62947200 & 0.84153300 \\ \mathrm{H} & -1.68773000 & -3.36132700 & -1.26982700 \\ \mathrm{H} & -3.68630500 & -4.74227300 & -1.78261900 \\ \mathrm{H} & -5.96994000 & -3.88896500 & -1.37108900 \\ \mathrm{H} & -6.34380500 & -1.61578200 & -0.42767400 \\ \mathrm{C} & 1.70327000 & 1.59240000 & -1.89298500 \\ \mathrm{C} & 3.42164400 & 2.49175100 & -0.42889400 \\ \mathrm{C} & 3.20234800 & 3.76067000 & -0.96102800 \\ \mathrm{C} & 2.23129800 & 3.95636100 & -1.94650600 \\ \mathrm{C} & 1.48724400 & 2.86985800 & -2.41126800 \\ \mathrm{C} & -1.43559400 & 2.34569100 & 0.33795400 \\ \mathrm{C} & -3.30234100 & 2.15074200 & 1.88078300 \\ \mathrm{C} & -1.11081600 & 3.58062400 & 0.89948300 \\ \mathrm{C} & -2.97378100 & 3.38993200 & 2.42447400 \\ \mathrm{C} & -1.87510900 & 4.10582700 & 1.94186500 \\ & 1.14703700 & 0.74268000 & -2.27168000 \\ \mathrm{H} & 4.17263200 & 2.31984000 & 0.33334200 \\ & 3.79133900 & 4.59956600 & -0.60118000\end{array}$




$\begin{array}{lrrr}\mathrm{H} & 2.06363400 & 4.94746600 & -2.35851600 \\ \mathrm{H} & 0.74598200 & 3.00956400 & -3.19300100 \\ \mathrm{H} & -0.85580800 & 1.95588300 & -0.48920400 \\ \mathrm{H} & -4.15082900 & 1.58164600 & 2.24171400 \\ \mathrm{H} & -0.26161100 & 4.13219100 & 0.50641400 \\ \mathrm{H} & -3.57614300 & 3.79410000 & 3.23321800 \\ \mathrm{H} & -1.62023400 & 5.06970000 & 2.37347600\end{array}$

\section{anti-14}

Point Group: CI

B3LYP/6-31G* $=-1296.74875$ au

B3LYP/6-31G* Zero Point Corrected Energy $=-1296.36021$ au $\mathrm{NIMAG}=0$

$\begin{array}{lrrr}\text { B3LYP/6-31G* Optimized Cartesian Coordinates } \\ \text { C } & -0.60562000 & 0.07366800 & 0.01564500 \\ \mathrm{C} & -2.00570700 & 0.13719600 & 0.03543700 \\ \mathrm{C} & -2.94500600 & -0.91585300 & 0.00873000 \\ \mathrm{C} & -2.85809600 & -2.32614500 & 0.02575500 \\ \mathrm{C} & -4.03459200 & -3.04244600 & -0.01799500 \\ \mathrm{C} & -4.21564000 & -0.26217900 & -0.05388300 \\ \mathrm{C} & -5.30325600 & -2.39150900 & -0.07929400 \\ \mathrm{C} & -5.41332400 & -1.02002900 & -0.09669600 \\ \mathrm{~N} & -4.08707300 & 1.07948200 & -0.05728300 \\ \mathrm{~N} & -2.75613900 & 1.29742400 & 0.00535900 \\ \mathrm{C} & -2.28753200 & 2.64437200 & 0.01164400 \\ \mathrm{H} & -1.89381200 & -2.82330500 & 0.07897500 \\ \mathrm{H} & -4.00397900 & -4.12841100 & -0.00202300 \\ \mathrm{H} & -6.20177600 & -3.00185400 & -0.11089300 \\ \mathrm{H} & -6.37514700 & -0.51896800 & -0.14049900 \\ \mathrm{C} & 0.60562000 & -0.07366800 & -0.01564500 \\ \mathrm{C} & 2.00570700 & -0.13719600 & -0.03543700 \\ \mathrm{C} & 2.94500600 & 0.91585300 & -0.00873000 \\ \mathrm{C} & 2.85809600 & 2.32614500 & -0.02575500 \\ \mathrm{C} & 4.03459200 & 3.04244600 & 0.01799500 \\ \mathrm{C} & 4.21564000 & 0.26217900 & 0.05388300 \\ \mathrm{C} & 5.30325600 & 2.39150900 & 0.07929400 \\ \mathrm{C} & 5.41332400 & 1.02002900 & 0.09669600 \\ \mathrm{~N} & 4.08707300 & -1.07948200 & 0.05728300 \\ \mathrm{~N} & 2.75613900 & -1.29742400 & -0.00535900 \\ \mathrm{C} & 2.28753200 & -2.64437200 & -0.01164400 \\ \mathrm{H} & 1.89381200 & 2.82330500 & -0.07897500 \\ \mathrm{H} & 4.00397900 & 4.12841100 & 0.00202300 \\ \mathrm{H} & 6.20177600 & 3.00185400 & 0.11089300 \\ \mathrm{H} & 6.37514700 & 0.51896800 & 0.14049900 \\ \mathrm{C} & -1.20677200 & 3.01858900 & 0.81537600 \\ \mathrm{C} & -2.95135800 & 3.58990400 & -0.77618300 \\ \mathrm{C} & -2.51593500 & 4.91314600 & -0.76922800 \\ \mathrm{C} & -1.42525200 & 5.29502200 & 0.01532200 \\ \mathrm{C} & -0.77629100 & 4.34575000 & 0.80666300 \\ \mathrm{C} & 1.20677200 & -3.01858900 & -0.81537600\end{array}$




$\begin{array}{lrrr}\mathrm{C} & 2.95135800 & -3.58990400 & 0.77618300 \\ \mathrm{C} & 0.77629100 & -4.34575000 & -0.80666300 \\ \mathrm{C} & 2.51593500 & -4.91314600 & 0.76922800 \\ \mathrm{C} & 1.42525200 & -5.29502200 & -0.01532200 \\ \mathrm{H} & -0.72133700 & 2.28812500 & 1.45176100 \\ \mathrm{H} & -3.79871300 & 3.27687000 & -1.37479200 \\ \mathrm{H} & -3.03028400 & 5.64697600 & -1.38326200 \\ \mathrm{H} & -1.08821200 & 6.32770600 & 0.01619000 \\ \mathrm{H} & 0.05906400 & 4.63772000 & 1.43696900 \\ \mathrm{H} & 0.72133700 & -2.28812500 & -1.45176100 \\ \mathrm{H} & 3.79871300 & -3.27687000 & 1.37479200 \\ \mathrm{H} & -0.05906400 & -4.63772000 & -1.43696900 \\ \mathrm{H} & 3.03028400 & -5.64697600 & 1.38326200 \\ \mathrm{H} & 1.08821200 & -6.32770600 & -0.01619000\end{array}$

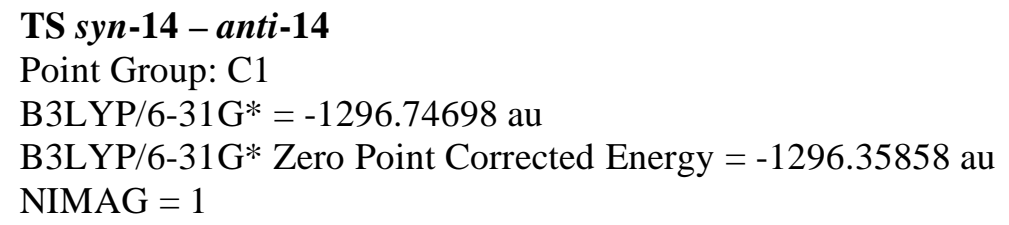

B3LYP/6-31G* Optimized Cartesian Coordinates

$\begin{array}{lrrr}\mathrm{C} & -0.63585800 & -0.08485700 & -0.40421600 \\ \mathrm{C} & -2.03176600 & -0.23841900 & -0.37724600 \\ \mathrm{C} & -2.83815000 & -1.18892500 & -1.03655200 \\ \mathrm{C} & -2.58494000 & -2.29668900 & -1.87710800 \\ \mathrm{C} & -3.66703900 & -3.00666000 & -2.34923700 \\ \mathrm{C} & -4.17827800 & -0.81932600 & -0.70319100 \\ \mathrm{C} & -5.00478000 & -2.64345600 & -2.00837800 \\ \mathrm{C} & -5.27746800 & -1.56581100 & -1.19731800 \\ \mathrm{~N} & -4.20924800 & 0.26597200 & 0.09676800 \\ \mathrm{~N} & -2.91662500 & 0.59323600 & 0.28544200 \\ \mathrm{C} & -2.61624500 & 1.73722900 & 1.08565300 \\ \mathrm{H} & -1.56817700 & -2.57194500 & -2.14244400 \\ \mathrm{H} & -3.50664700 & -3.86357800 & -2.99765600 \\ \mathrm{H} & -5.82369400 & -3.23705200 & -2.40554700 \\ \mathrm{H} & -6.29208900 & -1.28255900 & -0.93570400 \\ \mathrm{C} & 0.58170200 & -0.02748200 & -0.47046000 \\ \mathrm{C} & 1.96978300 & 0.15004000 & -0.59244800 \\ \mathrm{C} & 2.67738900 & 1.17134700 & -1.25854000 \\ \mathrm{C} & 2.30709200 & 2.28784600 & -2.04233500 \\ \mathrm{C} & 3.31394000 & 3.08904100 & -2.53409500 \\ \mathrm{C} & 4.05328500 & 0.89454800 & -0.98679700 \\ \mathrm{C} & 4.68848800 & 2.81018000 & -2.26731400 \\ \mathrm{C} & 5.07378500 & 1.73071500 & -1.50608400 \\ \mathrm{~N} & 4.19631600 & -0.20748600 & -0.22330200 \\ \mathrm{~N} & 2.93966800 & -0.64003300 & -0.00064300 \\ \mathrm{C} & 2.75614000 & -1.79072200 & 0.82349400 \\ \mathrm{H} & 1.26167300 & 2.50030100 & -2.24576800 \\ \mathrm{H} & 3.06413100 & 3.95463000 & -3.14136500 \\ \mathrm{H} & 5.44456500 & 3.47272000 & -2.67985400\end{array}$




$\begin{array}{lrrr}\mathrm{H} & 6.11687200 & 1.51291900 & -1.29949000 \\ \mathrm{C} & -1.51207700 & 1.74013500 & 1.94233400 \\ \mathrm{C} & -3.46758200 & 2.84467400 & 1.01391700 \\ \mathrm{C} & -3.19868700 & 3.96535500 & 1.79588700 \\ \mathrm{C} & -2.08736800 & 3.98687300 & 2.64203500 \\ \mathrm{C} & -1.24950000 & 2.87331100 & 2.71215800 \\ \mathrm{C} & 1.73773300 & -2.70974900 & 0.55623800 \\ \mathrm{C} & 3.63440100 & -1.99185500 & 1.89397300 \\ \mathrm{C} & 1.58669900 & -3.82228600 & 1.38417200 \\ \mathrm{C} & 3.47855500 & -3.11248900 & 2.70569900 \\ \mathrm{C} & 2.45229600 & -4.02777000 & 2.45896000 \\ \mathrm{H} & -0.87439500 & 0.86762000 & 2.01567700 \\ \mathrm{H} & -4.32621400 & 2.80852800 & 0.35392100 \\ \mathrm{H} & -3.85852700 & 4.82648400 & 1.73898400 \\ \mathrm{H} & -1.87905600 & 4.86478800 & 3.24700100 \\ \mathrm{H} & -0.39110700 & 2.87609800 & 3.37777600 \\ \mathrm{H} & 1.08073100 & -2.56534000 & -0.29250100 \\ \mathrm{H} & 4.42594700 & -1.27296200 & 2.06867500 \\ \mathrm{H} & 0.79435600 & -4.53560800 & 1.17609700 \\ \mathrm{H} & 4.15974500 & -3.26731900 & 3.53768200 \\ \mathrm{H} & 2.33180100 & -4.89832600 & 3.09744200\end{array}$

\section{syn-15a}

Point Group: C1

B3LYP/6-31G* $=-1296.64391 \mathrm{au}$

B3LYP/6-31G* Zero Point Corrected Energy $=-1296.26047$ au $\mathrm{NIMAG}=0$

B3LYP/6-31G* Optimized Cartesian Coordinates

$\begin{array}{lrrr}\mathrm{C} & 0.67449400 & -2.50610300 & 0.07343400 \\ \mathrm{C} & 1.88944000 & -2.48666800 & 0.20298900 \\ \mathrm{C} & 3.29611300 & -2.55570700 & 0.37590500 \\ \mathrm{C} & 3.89872900 & -3.78721000 & 0.71011100 \\ \mathrm{C} & 5.27325500 & -3.87783800 & 0.88753300 \\ \mathrm{C} & 4.12163400 & -1.41098700 & 0.22262800 \\ \mathrm{C} & 6.08053900 & -2.74146900 & 0.73488200 \\ \mathrm{C} & 5.50897100 & -1.51991000 & 0.40519700 \\ \mathrm{~N} & 3.45368400 & -0.21406100 & -0.11860400 \\ \mathrm{~N} & 4.19968500 & 0.79581900 & -0.24209500 \\ \mathrm{C} & 3.52176900 & 1.98960800 & -0.59177800 \\ \mathrm{H} & 3.26764300 & -4.66223400 & 0.82711400 \\ \mathrm{H} & 5.72106900 & -4.83359200 & 1.14497300 \\ \mathrm{H} & 7.15556000 & -2.81612200 & 0.87391700 \\ \mathrm{H} & 6.11229400 & -0.62782400 & 0.28034300 \\ \mathrm{C} & -0.67504500 & -2.50605100 & -0.07361800 \\ \mathrm{C} & -1.88997900 & -2.48659800 & -0.20328300 \\ \mathrm{C} & -3.29668300 & -2.55535000 & -0.37605500 \\ \mathrm{C} & -3.89958500 & -3.78669300 & -0.71032300 \\ \mathrm{C} & -5.27415600 & -3.87704100 & -0.88755000 \\ \mathrm{C} & -4.12195900 & -1.41047200 & -0.22258600 \\ \mathrm{C} & -6.08119800 & -2.74053500 & -0.73465800\end{array}$




$\begin{array}{lrrr}\mathrm{C} & -5.50934200 & -1.51911500 & -0.40495700 \\ \mathrm{~N} & -3.45374200 & -0.21371900 & 0.11870600 \\ \mathrm{~N} & -4.19948100 & 0.79638900 & 0.24193000 \\ \mathrm{C} & -3.52130700 & 1.98999700 & 0.59173600 \\ \mathrm{H} & -3.26868600 & -4.66182500 & -0.82753000 \\ \mathrm{H} & -5.72218700 & -4.83268900 & -1.14500900 \\ \mathrm{H} & -7.15626000 & -2.81496900 & -0.87350000 \\ \mathrm{H} & -6.11247600 & -0.62692200 & -0.27993900 \\ \mathrm{C} & -4.33086500 & 3.12728500 & 0.71664800 \\ \mathrm{C} & -2.13560700 & 2.08603100 & 0.81461200 \\ \mathrm{C} & -3.76694600 & 4.35490100 & 1.05969200 \\ \mathrm{C} & -1.58027700 & 3.31324000 & 1.15754000 \\ \mathrm{C} & -2.39175700 & 4.44960000 & 1.28021000 \\ \mathrm{C} & 4.33165900 & 3.12661100 & -0.71713800 \\ \mathrm{C} & 3.76801600 & 4.35437300 & -1.06010400 \\ \mathrm{C} & 2.39276700 & 4.44950900 & -1.28007500 \\ \mathrm{C} & 1.58095400 & 3.31344000 & -1.15694200 \\ \mathrm{C} & 2.13601300 & 2.08608200 & -0.81410700 \\ \mathrm{H} & -5.39701300 & 3.02160900 & 0.54001000 \\ \mathrm{H} & -1.52676600 & 1.19389300 & 0.71779900 \\ \mathrm{H} & -4.39790200 & 5.23412900 & 1.15577800 \\ \mathrm{H} & -0.51019900 & 3.39005200 & 1.33022300 \\ \mathrm{H} & -1.94876200 & 5.40458600 & 1.55009700 \\ \mathrm{H} & 5.39783900 & 3.02059600 & -0.54089800 \\ \mathrm{H} & 4.39922900 & 5.23337500 & -1.15656200 \\ \mathrm{H} & 1.94998700 & 5.40461600 & -1.54988800 \\ \mathrm{H} & 0.51082600 & 3.39059100 & -1.32916200 \\ \mathrm{H} & 1.52690900 & 1.19415900 & -0.71697000\end{array}$

\section{anti-15a}

Point Group: CI

B3LYP/6-31G* $=-1296.64307 \mathrm{au}$

B3LYP/6-31G* Zero Point Corrected Energy $=-1296.25982 \mathrm{au}$ $\mathrm{NIMAG}=0$

B3LYP/6-31G* Optimized Cartesian Coordinates

$\begin{array}{llll}\mathrm{C} & 0.46514100 & -0.49429300 & 0.00289300 \\ \mathrm{C} & 1.31414300 & -1.37337100 & 0.00813800 \\ \mathrm{C} & 2.22112100 & -2.46449000 & 0.01413100 \\ \mathrm{C} & 1.72108400 & -3.78440600 & 0.01379000 \\ \mathrm{C} & 2.58701000 & -4.87009000 & 0.01970800 \\ \mathrm{C} & 3.62695700 & -2.26731100 & 0.02055700 \\ \mathrm{C} & 3.97425200 & -4.66530400 & 0.02612300 \\ \mathrm{C} & 4.48815600 & -3.37562200 & 0.02653400 \\ \mathrm{~N} & 4.05982100 & -0.92259300 & 0.02045600 \\ \mathrm{~N} & 5.31189400 & -0.76753400 & 0.02584600 \\ \mathrm{C} & 5.73919500 & 0.58279400 & 0.02565600 \\ \mathrm{H} & 0.64637500 & -3.93464200 & 0.00884400 \\ \mathrm{H} & 2.18533500 & -5.87952400 & 0.01938800 \\ \mathrm{H} & 4.64910100 & -5.51686800 & 0.03078700 \\ \mathrm{H} & 5.55663000 & -3.19196400 & 0.03140400\end{array}$




$\begin{array}{lrrr}\mathrm{C} & -0.46514100 & 0.49429300 & -0.00289300 \\ \mathrm{C} & -1.31414300 & 1.37337100 & -0.00813800 \\ \mathrm{C} & -2.22112100 & 2.46449000 & -0.01413100 \\ \mathrm{C} & -1.72108400 & 3.78440600 & -0.01379000 \\ \mathrm{C} & -2.58701000 & 4.87009000 & -0.01970800 \\ \mathrm{C} & -3.62695700 & 2.26731100 & -0.02055700 \\ \mathrm{C} & -3.97425200 & 4.66530400 & -0.02612300 \\ \mathrm{C} & -4.48815600 & 3.37562200 & -0.02653400 \\ \mathrm{~N} & -4.05982100 & 0.92259300 & -0.02045600 \\ \mathrm{~N} & -5.31189400 & 0.76753400 & -0.02584600 \\ \mathrm{C} & -5.73919500 & -0.58279400 & -0.02565600 \\ \mathrm{H} & -0.64637500 & 3.93464200 & -0.00884400 \\ \mathrm{H} & -2.18533500 & 5.87952400 & -0.01938800 \\ \mathrm{H} & -4.64910100 & 5.51686800 & -0.03078700 \\ \mathrm{H} & -5.55663000 & 3.19196400 & -0.03140400 \\ \mathrm{C} & 7.12765300 & 0.77434000 & 0.03235200 \\ \mathrm{C} & 4.87648200 & 1.69370100 & 0.01931400 \\ \mathrm{C} & 7.65742400 & 2.06337400 & 0.03283400 \\ \mathrm{C} & 5.41235800 & 2.97568600 & 0.01979200 \\ \mathrm{C} & 6.80075300 & 3.16558500 & 0.02655200 \\ \mathrm{H} & 7.76779600 & -0.10271600 & 0.03712100 \\ \mathrm{H} & 3.80548700 & 1.52397500 & 0.01415900 \\ \mathrm{H} & 8.73419000 & 2.20781400 & 0.03806100 \\ \mathrm{H} & 4.74934900 & 3.83687400 & 0.01488700 \\ \mathrm{H} & 7.20984400 & 4.17238500 & 0.02686300 \\ \mathrm{C} & -4.87648200 & -1.69370100 & -0.01931400 \\ \mathrm{C} & -5.41235800 & -2.97568600 & -0.01979200 \\ \mathrm{C} & -6.80075300 & -3.16558500 & -0.02655200 \\ \mathrm{C} & -7.65742400 & -2.06337400 & -0.03283400 \\ \mathrm{C} & -7.12765300 & -0.77434000 & -0.03235200 \\ \mathrm{H} & -3.80548700 & -1.52397500 & -0.01415900 \\ \mathrm{H} & -4.74934900 & -3.83687400 & -0.01488700 \\ \mathrm{H} & -7.20984400 & -4.17238500 & -0.02686300 \\ \mathrm{H} & -8.73419000 & -2.20781400 & -0.03806100 \\ \mathrm{H} & -7.76779600 & 0.10271600 & -0.03712100 \\ & & & \\ & & & \end{array}$

\section{TS syn-15a - anti-15a}

Point Group: C1

B3LYP/6-31G* $=-1296.64280$ au

B3LYP/6-31 G* Zero Point Corrected Energy $=-1296.25948$ au $\mathrm{NIMAG}=1$

B3LYP/6-31G* Optimized Cartesian Coordinates

$\begin{array}{llll}\mathrm{C} & -0.58355400 & -0.34755200 & -1.66037900 \\ \mathrm{C} & -1.63893700 & -0.96300500 & -1.65205700 \\ \mathrm{C} & -2.82378000 & -1.74377700 & -1.71440300 \\ \mathrm{C} & -2.86543800 & -2.88099500 & -2.54822500 \\ \mathrm{C} & -4.01379900 & -3.65862200 & -2.62775500 \\ \mathrm{C} & -3.97636900 & -1.40922200 & -0.95756100 \\ \mathrm{C} & -5.14825800 & -3.32002000 & -1.87670000 \\ \mathrm{C} & -5.12866600 & -2.20528700 & -1.04929300\end{array}$




$\begin{array}{lrrr}\mathrm{N} & -3.86273200 & -0.26094200 & -0.14204300 \\ \mathrm{~N} & -4.89878800 & 0.02802100 & 0.51698800 \\ \mathrm{C} & -4.77637100 & 1.17769900 & 1.33539600 \\ \mathrm{H} & -1.98380900 & -3.13677400 & -3.12698000 \\ \mathrm{H} & -4.02922900 & -4.53114700 & -3.27490200 \\ \mathrm{H} & -6.04471900 & -3.93058700 & -1.94134500 \\ \mathrm{H} & -5.99285200 & -1.92348600 & -0.45837400 \\ \mathrm{C} & 0.58355500 & 0.34755900 & -1.66037800 \\ \mathrm{C} & 1.63893800 & 0.96301200 & -1.65205500 \\ \mathrm{C} & 2.82378200 & 1.74378300 & -1.71439700 \\ \mathrm{C} & 2.86544300 & 2.88100200 & -2.54821700 \\ \mathrm{C} & 4.01380400 & 3.65862900 & -2.62774200 \\ \mathrm{C} & 3.97636900 & 1.40922500 & -0.95755300 \\ \mathrm{C} & 5.14826200 & 3.32002500 & -1.87668500 \\ \mathrm{C} & 5.12866600 & 2.20529000 & -1.04928100 \\ \mathrm{~N} & 3.86272900 & 0.26094600 & -0.14203700 \\ \mathrm{~N} & 4.89878900 & -0.02802800 & 0.51698400 \\ \mathrm{C} & 4.77636900 & -1.17770500 & 1.33539300 \\ \mathrm{H} & 1.98381600 & 3.13678300 & -3.12697400 \\ \mathrm{H} & 4.02923700 & 4.53115500 & -3.27488800 \\ \mathrm{H} & 6.04472300 & 3.93059200 & -1.94132600 \\ \mathrm{H} & 5.99285100 & 1.92348800 & -0.45835900 \\ \mathrm{C} & -5.92161200 & 1.51881400 & 2.06799100 \\ \mathrm{C} & -3.61218500 & 1.95927900 & 1.44626700 \\ \mathrm{C} & -5.91121300 & 2.63186500 & 2.90661100 \\ \mathrm{C} & -3.60950500 & 3.06716200 & 2.28489600 \\ \mathrm{C} & -4.75547300 & 3.40715100 & 3.01627500 \\ \mathrm{H} & -6.80458200 & 0.89584200 & 1.96169500 \\ \mathrm{H} & -2.73614200 & 1.67871300 & 0.87219600 \\ \mathrm{H} & -6.80077100 & 2.89348200 & 3.47282800 \\ \mathrm{H} & -2.71168400 & 3.67303600 & 2.37401100 \\ \mathrm{H} & -4.74318500 & 4.27542600 & 3.66972500 \\ \mathrm{C} & 3.61217500 & -1.95927100 & 1.44628100 \\ \mathrm{C} & 3.60949400 & -3.06715400 & 2.28490900 \\ \mathrm{C} & 4.75546900 & -3.40715900 & 3.01627100 \\ \mathrm{C} & 5.91121700 & -2.63188700 & 2.90659000 \\ \mathrm{C} & 5.92161700 & -1.51883600 & 2.06797000 \\ \mathrm{H} & 2.73612700 & -1.67869400 & 0.87222300 \\ \mathrm{H} & 2.71166700 & -3.67301700 & 2.37403700 \\ \mathrm{H} & 4.74318000 & -4.27543400 & 3.66972000 \\ \mathrm{H} & 6.80078000 & -2.89351600 & 3.47279300 \\ \mathrm{H} & 6.80459300 & -0.89587400 & 1.96166200 \\ & & & \end{array}$

15b

Point Group: CI

B3LYP/6-31G* = -1296.63587 au

B3LYP/6-31G* Zero Point Corrected Energy $=-1296.25340 \mathrm{au}$ $\mathrm{NIMAG}=0$

B3LYP/6-31G* Optimized Cartesian Coordinates
$\mathrm{C}$
$\begin{array}{lll}0.49150000 & 0.46566700 & -0.01426600\end{array}$ 


\begin{tabular}{|c|c|c|c|}
\hline $\mathrm{C}$ & 1.41336300 & 1.27128400 & -0.04060100 \\
\hline $\mathrm{C}$ & 2.31398700 & 2.36511300 & -0.06915900 \\
\hline $\mathrm{C}$ & 1.75484600 & 3.66181800 & -0.08854100 \\
\hline $\mathrm{C}$ & 2.55016400 & 4.80216500 & -0.11185800 \\
\hline $\mathrm{C}$ & 3.73717800 & 2.25365100 & -0.07383800 \\
\hline $\mathrm{C}$ & 3.94310900 & 4.68350400 & -0.11400400 \\
\hline $\mathrm{C}$ & 4.51886000 & 3.41878900 & -0.09328800 \\
\hline $\mathrm{N}$ & 4.49808100 & 1.06653700 & -0.05606100 \\
\hline $\mathrm{N}$ & 3.83745800 & -0.00710700 & -0.07904400 \\
\hline $\mathrm{C}$ & 4.61698400 & -1.18921000 & -0.05643300 \\
\hline $\mathrm{H}$ & 0.67300300 & 3.75032800 & -0.08439300 \\
\hline $\mathrm{H}$ & 2.08232200 & 5.78254400 & -0.12683300 \\
\hline $\mathrm{H}$ & 4.57161900 & 5.56904800 & -0.13078100 \\
\hline $\mathrm{H}$ & 5.59645600 & 3.28899300 & -0.09309600 \\
\hline $\mathrm{C}$ & -0.49150000 & -0.46566700 & 0.01426600 \\
\hline $\mathrm{C}$ & -1.41336300 & -1.27128400 & 0.04060100 \\
\hline $\mathrm{C}$ & -2.31398700 & -2.36511300 & 0.06915900 \\
\hline $\mathrm{C}$ & -1.75484600 & -3.66181800 & 0.08854100 \\
\hline $\mathrm{C}$ & -2.55016400 & -4.80216500 & 0.11185800 \\
\hline $\mathrm{C}$ & -3.73717800 & -2.25365100 & 0.07383800 \\
\hline $\mathrm{C}$ & -3.94310900 & -4.68350400 & 0.11400400 \\
\hline $\mathrm{C}$ & -4.51886000 & -3.41878900 & 0.09328800 \\
\hline $\mathrm{N}$ & -4.49808100 & -1.06653700 & 0.05606100 \\
\hline $\mathrm{N}$ & -3.83745800 & 0.00710700 & 0.07904400 \\
\hline $\mathrm{C}$ & -4.61698400 & 1.18921000 & 0.05643300 \\
\hline $\mathrm{H}$ & -0.67300300 & -3.75032800 & 0.08439300 \\
\hline $\mathrm{H}$ & -2.08232200 & -5.78254400 & 0.12683300 \\
\hline $\mathrm{H}$ & -4.57161900 & -5.56904800 & 0.13078100 \\
\hline $\mathrm{H}$ & -5.59645600 & -3.28899300 & 0.09309600 \\
\hline $\mathrm{C}$ & -6.02166800 & 1.22481000 & 0.00237200 \\
\hline $\mathrm{C}$ & -3.88688000 & 2.38555100 & 0.09132700 \\
\hline $\mathrm{C}$ & -6.67626500 & 2.45047900 & -0.01539400 \\
\hline $\mathrm{C}$ & -4.55067400 & 3.61082900 & 0.07341400 \\
\hline $\mathrm{C}$ & -5.94531700 & 3.64534200 & 0.01998300 \\
\hline $\mathrm{C}$ & 3.88688000 & -2.38555100 & -0.09132700 \\
\hline $\mathrm{C}$ & 4.55067400 & -3.61082900 & -0.07341400 \\
\hline $\mathrm{C}$ & 5.94531700 & -3.64534200 & -0.01998300 \\
\hline $\mathrm{C}$ & 6.67626500 & -2.45047900 & 0.01539400 \\
\hline $\mathrm{C}$ & 6.02166800 & -1.22481000 & -0.00237200 \\
\hline $\mathrm{H}$ & -6.56891900 & 0.28917800 & -0.02457200 \\
\hline $\mathrm{H}$ & -2.80367800 & 2.32384800 & 0.13199600 \\
\hline $\mathrm{H}$ & -7.76192200 & 2.48200300 & -0.05720300 \\
\hline $\mathrm{H}$ & -3.98255100 & 4.53653200 & 0.10088600 \\
\hline $\mathrm{H}$ & -6.46583600 & 4.59923600 & 0.00558900 \\
\hline $\mathrm{H}$ & 2.80367800 & -2.32384800 & -0.13199600 \\
\hline $\mathrm{H}$ & 3.98255100 & -4.53653200 & -0.10088600 \\
\hline $\mathrm{H}$ & 6.46583600 & -4.59923600 & -0.00558900 \\
\hline $\mathrm{H}$ & 7.76192200 & -2.48200300 & 0.05720300 \\
\hline $\mathrm{H}$ & 6.56891900 & -0.28917800 & 0.02457200 \\
\hline
\end{tabular}




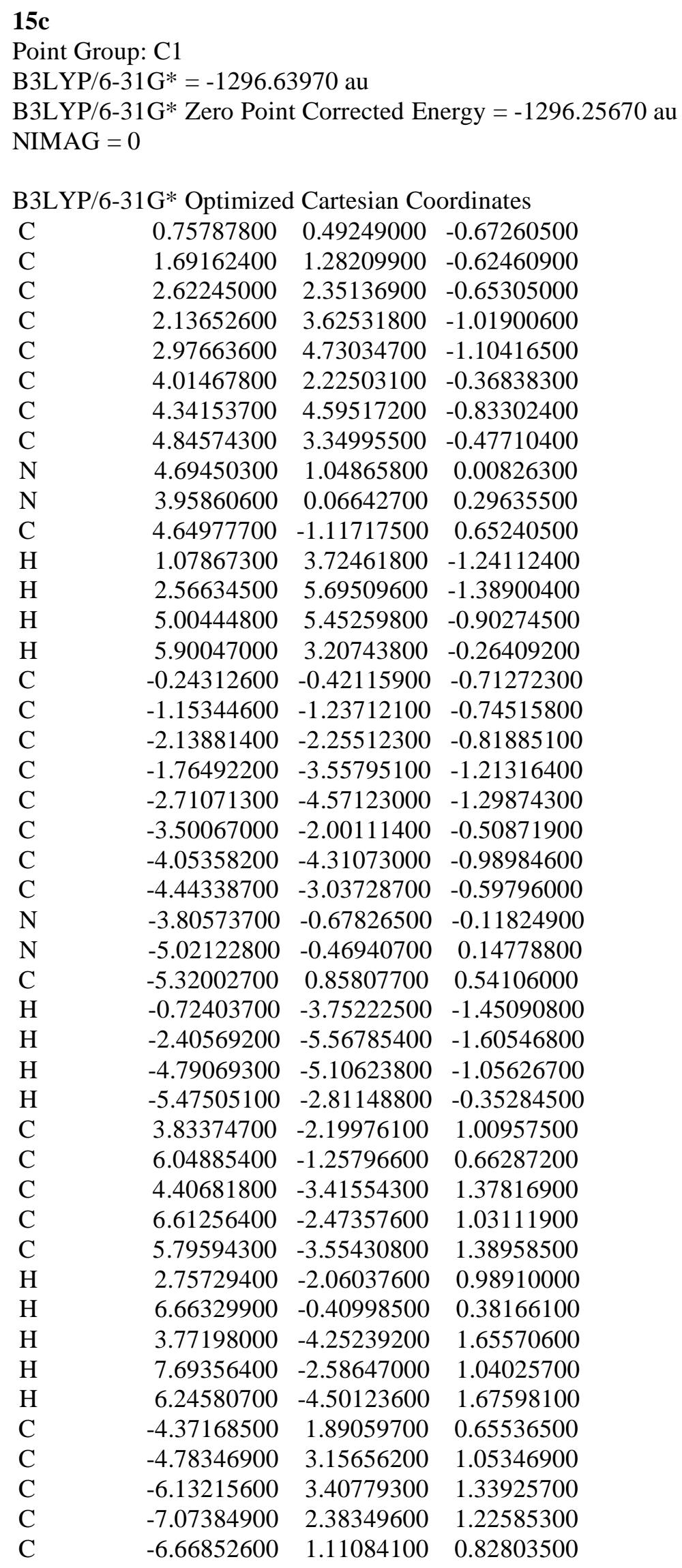




$\begin{array}{llll}\mathrm{H} & -3.33328600 & 1.67465100 & 0.42981700 \\ \mathrm{H} & -4.05366800 & 3.95678400 & 1.14434400 \\ \mathrm{H} & -6.44413800 & 4.40131900 & 1.65007900 \\ \mathrm{H} & -8.11997500 & 2.57574700 & 1.44722700 \\ \mathrm{H} & -7.37676700 & 0.29350600 & 0.73124800\end{array}$

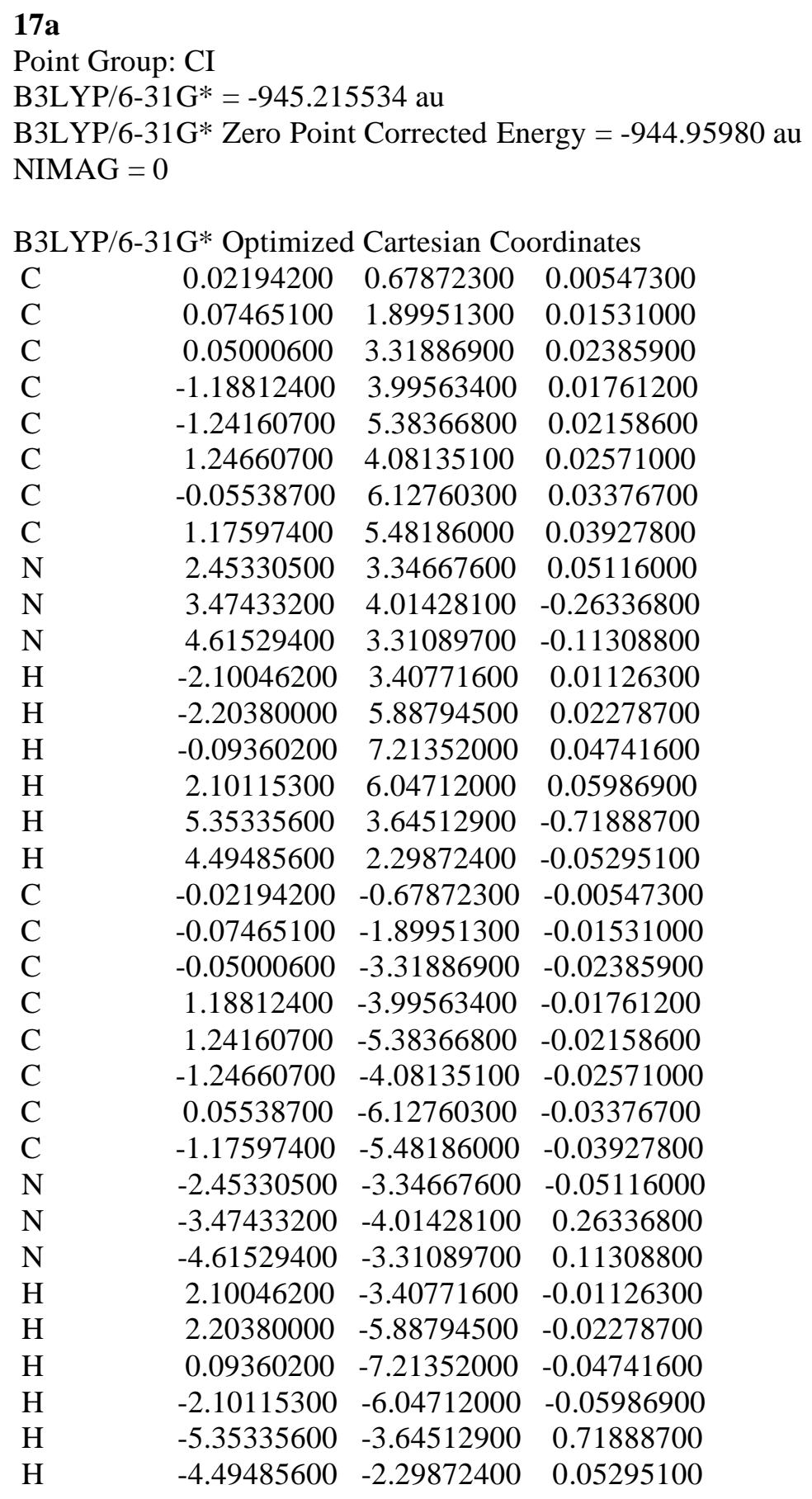

17b

Point Group: CI 


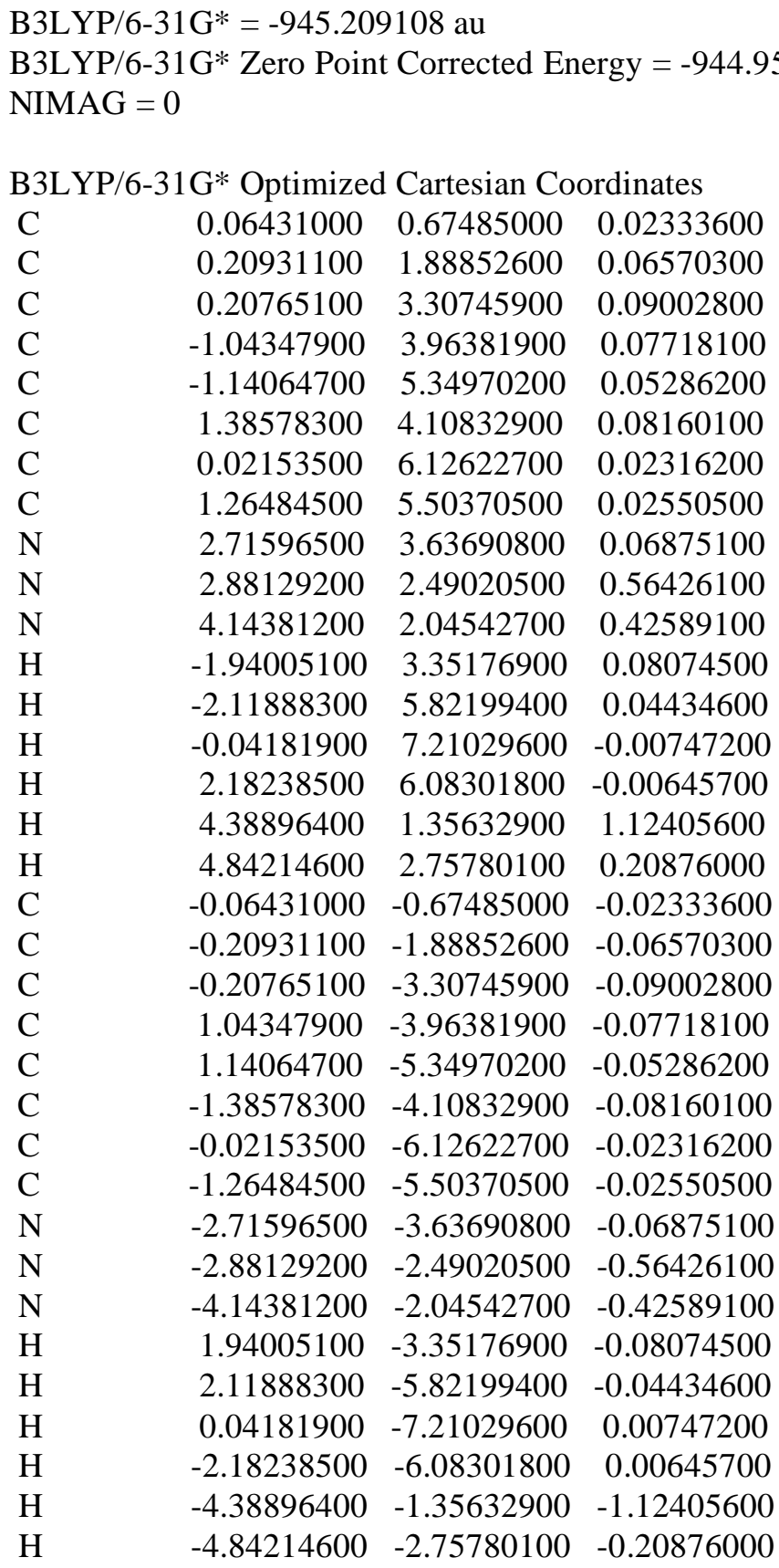

$17 \mathrm{c}$

Point Group: C1

B3LYP/6-31G* $=-945.212545 \mathrm{au}$

B3LYP/6-31G* Zero Point Corrected Energy $=-944.95691 \mathrm{au}$ $\mathrm{NIMAG}=0$

B3LYP/6-31G* Optimized Cartesian Coordinates

$\begin{array}{llll}\mathrm{C} & 0.82789600 & -0.73932000 & 0.07337400 \\ \mathrm{C} & 2.04407400 & -0.64061500 & 0.15038200 \\ \mathrm{C} & 3.45579800 & -0.66687600 & 0.30508400 \\ \mathrm{C} & 4.02166700 & -1.72860500 & 1.04420400\end{array}$




$\begin{array}{lrrr}\mathrm{C} & 5.39700200 & -1.85526500 & 1.19987100 \\ \mathrm{C} & 4.33457400 & 0.28504000 & -0.28594600 \\ \mathrm{C} & 6.25196900 & -0.92601600 & 0.60026600 \\ \mathrm{C} & 5.71856800 & 0.12188200 & -0.14257800 \\ \mathrm{~N} & 3.94564000 & 1.38044500 & -1.08881000 \\ \mathrm{~N} & 2.79807200 & 1.83468300 & -0.83885900 \\ \mathrm{~N} & 2.47320100 & 2.88559500 & -1.61595700 \\ \mathrm{H} & 3.35062600 & -2.45659000 & 1.48944700 \\ \mathrm{H} & 5.80051200 & -2.68313800 & 1.77589500 \\ \mathrm{H} & 7.32886800 & -1.02125500 & 0.70691000 \\ \mathrm{H} & 6.36089800 & 0.85113400 & -0.62639300 \\ \mathrm{H} & 1.47181800 & 3.00698300 & -1.68980400 \\ \mathrm{H} & 3.01271700 & 2.97976200 & -2.47727000 \\ \mathrm{C} & -0.52399300 & -0.81453600 & -0.03194600 \\ \mathrm{C} & -1.74145800 & -0.86468100 & -0.12548900 \\ \mathrm{C} & -3.14897200 & -0.99551900 & -0.26465600 \\ \mathrm{C} & -3.69416400 & -2.19981600 & -0.75707800 \\ \mathrm{C} & -5.06715500 & -2.35284900 & -0.90432500 \\ \mathrm{C} & -4.02880000 & 0.05902300 & 0.09128600 \\ \mathrm{C} & -5.92671200 & -1.30202500 & -0.56190700 \\ \mathrm{C} & -5.41172500 & -0.10710100 & -0.07163200 \\ \mathrm{~N} & -3.42131400 & 1.24336500 & 0.56666800 \\ \mathrm{~N} & -4.20588600 & 2.02333000 & 1.16893400 \\ \mathrm{~N} & -3.61429400 & 3.18765700 & 1.50829800 \\ \mathrm{H} & -3.01699700 & -3.00545100 & -1.02245700 \\ \mathrm{H} & -5.46914900 & -3.28469200 & -1.29175700 \\ \mathrm{H} & -7.00049100 & -1.41448900 & -0.68531700 \\ \mathrm{H} & -6.06715000 & 0.71674600 & 0.18842900 \\ \mathrm{H} & -4.05769000 & 3.62091400 & 2.30792300 \\ \mathrm{H} & -2.59332100 & 3.16822800 & 1.50900600\end{array}$

\section{$18 b$}

Point Group: $\mathrm{C} 1$

B3LYP/6-31G* $=-945.179151$ au

B3LYP/6-31G* Zero Point Corrected Energy = -944.925593 au NIMAG $=1$

B3LYP/6-31G* Optimized Cartesian Coordinates

$\begin{array}{lrrr}\mathrm{C} & -0.48645700 & -0.06771700 & -0.65537900 \\ \mathrm{C} & -1.71239100 & -0.17951100 & -0.52669300 \\ \mathrm{C} & -3.07790800 & -0.51607900 & -0.40755500 \\ \mathrm{C} & -3.47161000 & -1.84317400 & -0.70738400 \\ \mathrm{C} & -4.80431200 & -2.23072200 & -0.67721500 \\ \mathrm{C} & -4.09918200 & 0.41537100 & -0.05129200 \\ \mathrm{C} & -5.79800900 & -1.30557400 & -0.33609800 \\ \mathrm{C} & -5.43827700 & 0.00205100 & -0.03334100 \\ \mathrm{~N} & -3.88544200 & 1.77288000 & 0.25006200 \\ \mathrm{~N} & -2.70669700 & 2.05609400 & 0.59040900 \\ \mathrm{~N} & -2.54307900 & 3.37197000 & 0.86646300 \\ \mathrm{H} & -2.70046400 & -2.55760700 & -0.97892600 \\ \mathrm{H} & -5.06971400 & -3.25558900 & -0.92191900\end{array}$




$\begin{array}{lrrr}\mathrm{H} & -6.84213400 & -1.60348300 & -0.30915000 \\ \mathrm{H} & -6.18530400 & 0.74469000 & 0.22966400 \\ \mathrm{H} & -1.58008400 & 3.65671400 & 0.73993200 \\ \mathrm{H} & -3.24441700 & 3.98559100 & 0.44936800 \\ \mathrm{C} & 0.83123600 & 0.13600700 & -0.83281300 \\ \mathrm{C} & 2.00576500 & -0.11178900 & -0.37248000 \\ \mathrm{C} & 3.41740600 & 0.04922900 & -0.54333600 \\ \mathrm{C} & 4.09280200 & 0.58070800 & -1.65014900 \\ \mathrm{C} & 5.48185100 & 0.67768500 & -1.61364600 \\ \mathrm{C} & 4.16653500 & -0.38318800 & 0.57753400 \\ \mathrm{C} & 6.21246900 & 0.24549200 & -0.49398000 \\ \mathrm{C} & 5.55777100 & -0.28649600 & 0.61370600 \\ \mathrm{~N} & 3.39543000 & -0.87449000 & 1.64418100 \\ \mathrm{~N} & 2.20922200 & -0.96358900 & 1.22086700 \\ \mathrm{~N} & 1.21057300 & -1.32170600 & 2.05273200 \\ \mathrm{H} & 3.52705000 & 0.91801000 & -2.51293900 \\ \mathrm{H} & 6.00873300 & 1.09904700 & -2.46528900 \\ \mathrm{H} & 7.29535500 & 0.32784500 & -0.49248400 \\ \mathrm{H} & 6.09935100 & -0.62702000 & 1.49070300 \\ \mathrm{H} & 0.31691000 & -1.05613700 & 1.64460100 \\ \mathrm{H} & 1.34854500 & -1.01392700 & 3.01415200\end{array}$

\section{$18 c$}

Point Group: C1

B3LYP/6-31G* $=-945.185622 \mathrm{au}$

B3LYP/6-31G* Zero Point Corrected Energy $=-944.93100 \mathrm{au}$ NIMAG $=1$

B3LYP/6-31G* Optimized Cartesian Coordinates

$\begin{array}{lrrr}\mathrm{C} & 0.41869200 & -1.50662200 & -0.09629200 \\ \mathrm{C} & 1.64857200 & -1.46006800 & -0.02790700 \\ \mathrm{C} & 3.02995700 & -1.15184900 & 0.04895300 \\ \mathrm{C} & 3.99489600 & -2.17318100 & 0.17483300 \\ \mathrm{C} & 5.35142100 & -1.87629000 & 0.23124700 \\ \mathrm{C} & 3.47487800 & 0.19873300 & -0.02092800 \\ \mathrm{C} & 5.77443000 & -0.54389500 & 0.16146700 \\ \mathrm{C} & 4.84449100 & 0.48477600 & 0.03961400 \\ \mathrm{~N} & 2.47550400 & 1.19778500 & -0.09983000 \\ \mathrm{~N} & 2.86661100 & 2.27743200 & -0.62527400 \\ \mathrm{~N} & 1.93643400 & 3.24564600 & -0.59812400 \\ \mathrm{H} & 3.65005500 & -3.20120300 & 0.22331500 \\ \mathrm{H} & 6.07912400 & -2.67631800 & 0.33297300 \\ \mathrm{H} & 6.83358400 & -0.30581600 & 0.21226500 \\ \mathrm{H} & 5.16343500 & 1.52066700 & -0.00141100 \\ \mathrm{H} & 2.10333600 & 3.95809100 & -1.29551800 \\ \mathrm{H} & 0.96767900 & 2.95073300 & -0.44865800 \\ \mathrm{C} & -0.93294400 & -1.61570800 & -0.14974600 \\ \mathrm{C} & -1.93793400 & -0.80205100 & -0.09650400 \\ \mathrm{C} & -3.36238800 & -0.69875300 & -0.13880600 \\ \mathrm{C} & -4.29001200 & -1.68962900 & -0.49033500 \\ \mathrm{C} & -5.64888300 & -1.39331100 & -0.43228400\end{array}$




$\begin{array}{lrrc}\mathrm{C} & -3.83320900 & 0.57666000 & 0.25624300 \\ \mathrm{C} & -6.10302100 & -0.12210500 & -0.03700300 \\ \mathrm{C} & -5.19663100 & 0.87305600 & 0.31483300 \\ \mathrm{~N} & -2.82513500 & 1.48225600 & 0.60831500 \\ \mathrm{~N} & -1.72249600 & 0.94565000 & 0.29307600 \\ \mathrm{~N} & -0.56298300 & 1.57492200 & 0.61812200 \\ \mathrm{H} & -3.93806500 & -2.67222200 & -0.78806700 \\ \mathrm{H} & -6.37271600 & -2.16104900 & -0.69160700 \\ \mathrm{H} & -7.16912700 & 0.08234900 & -0.00408000 \\ \mathrm{H} & -5.52146500 & 1.86146800 & 0.62468700 \\ \mathrm{H} & 0.24101100 & 0.95036600 & 0.50699700 \\ \mathrm{H} & -0.60627600 & 2.02853200 & 1.53080200\end{array}$

\section{9b}

Point Group: $\mathrm{C} 1$

B3LYP/6-31G* $=-945.201928$ au

B3LYP/6-31G* Zero Point Corrected Energy = $-944.945385 \mathrm{au}$ $\mathrm{NIMAG}=0$

$\begin{array}{lrrr}\text { B3LYP/6-31G* Optimized Cartesian Coordinates } \\ \text { C } & -0.77081000 & 1.53903000 & 0.35859000 \\ \mathrm{C} & -2.03712000 & 1.03246000 & 0.13842000 \\ \mathrm{C} & -2.65279000 & -0.17958000 & -0.32772000 \\ \mathrm{C} & -2.16759000 & -1.39600000 & -0.82766000 \\ \mathrm{C} & -3.09419000 & -2.37358000 & -1.16787000 \\ \mathrm{C} & -4.04931000 & 0.02730000 & -0.18093000 \\ \mathrm{C} & -4.48491000 & -2.16104000 & -1.01825000 \\ \mathrm{C} & -4.98135000 & -0.96440000 & -0.52458000 \\ \mathrm{~N} & -4.31955000 & 1.28775000 & 0.31196000 \\ \mathrm{~N} & -3.14743000 & 1.82461000 & 0.48385000 \\ \mathrm{~N} & -3.02709000 & 3.13842000 & 0.89534000 \\ \mathrm{H} & -1.10074000 & -1.55947000 & -0.94892000 \\ \mathrm{H} & -2.74715000 & -3.32473000 & -1.56245000 \\ \mathrm{H} & -5.17380000 & -2.95254000 & -1.29931000 \\ \mathrm{H} & -6.04577000 & -0.78738000 & -0.40658000 \\ \mathrm{H} & -2.02472000 & 3.25371000 & 1.08834000 \\ \mathrm{H} & -3.59343000 & 3.26554000 & 1.73196000 \\ \mathrm{C} & 0.42554000 & 1.01501000 & 0.13397000 \\ \mathrm{C} & 1.59832000 & 0.58191000 & -0.00441000 \\ \mathrm{C} & 2.98183000 & 0.51799000 & -0.23713000 \\ \mathrm{C} & 3.63555000 & 1.55036000 & -0.95574000 \\ \mathrm{C} & 4.98038000 & 1.45130000 & -1.27756000 \\ \mathrm{C} & 3.76060000 & -0.60381000 & 0.18077000 \\ \mathrm{C} & 5.73197000 & 0.33933000 & -0.86490000 \\ \mathrm{C} & 5.12213000 & -0.67711000 & -0.14187000 \\ \mathrm{~N} & 3.18544000 & -1.66871000 & 0.87634000 \\ \mathrm{~N} & 2.09827000 & -1.35134000 & 1.43450000 \\ \mathrm{~N} & 1.45296000 & -2.38974000 & 2.00765000 \\ \mathrm{H} & 3.05409000 & 2.41106000 & -1.27067000 \\ \mathrm{H} & 5.45414000 & 2.24658000 & -1.84650000 \\ \mathrm{H} & 6.78884000 & 0.27510000 & -1.10683000\end{array}$




$\begin{array}{llll}\mathrm{H} & 5.67911000 & -1.54720000 & 0.19181000 \\ \mathrm{H} & 0.47170000 & -2.18443000 & 2.14553000 \\ \mathrm{H} & 1.66779000 & -3.30672000 & 1.61637000\end{array}$

19c

Point Group: C1

B3LYP/6-31G* $=-945.200995 \mathrm{au}$

B3LYP/6-31G* Zero Point Corrected Energy = -944.94413 au

$\mathrm{NIMAG}=0$

B3LYP/6-31G* Optimized Cartesian Coordinates

$\begin{array}{lrrr}\mathrm{C} & -0.39768300 & -1.39221600 & 0.13035500 \\ \mathrm{C} & -1.64420200 & -1.47726600 & 0.08879100 \\ \mathrm{C} & -3.01425100 & -1.15139700 & -0.02953900 \\ \mathrm{C} & -3.98730100 & -2.16754100 & -0.16733500 \\ \mathrm{C} & -5.33765800 & -1.86024100 & -0.26377300 \\ \mathrm{C} & -3.45240600 & 0.20613200 & 0.02209500 \\ \mathrm{C} & -5.75148600 & -0.52317900 & -0.19630900 \\ \mathrm{C} & -4.81955800 & 0.50073000 & -0.05232300 \\ \mathrm{~N} & -2.44662100 & 1.19005500 & 0.09836900 \\ \mathrm{~N} & -2.81053100 & 2.27756000 & 0.63097600 \\ \mathrm{~N} & -1.86153700 & 3.22223100 & 0.58756100 \\ \mathrm{H} & -3.64773200 & -3.19804000 & -0.19498600 \\ \mathrm{H} & -6.07034500 & -2.65333800 & -0.38129500 \\ \mathrm{H} & -6.80838300 & -0.27876900 & -0.26310200 \\ \mathrm{H} & -5.13412600 & 1.53807400 & -0.01327400 \\ \mathrm{H} & -2.00551100 & 3.94972800 & 1.27415800 \\ \mathrm{H} & -0.90108800 & 2.90783300 & 0.41426000 \\ \mathrm{C} & 0.93513100 & -1.52027200 & 0.25063900 \\ \mathrm{C} & 1.90089800 & -0.55155800 & 0.04290500 \\ \mathrm{C} & 3.32910300 & -0.65285200 & 0.12664100 \\ \mathrm{C} & 4.20037500 & -1.69040400 & 0.48618600 \\ \mathrm{C} & 5.56295800 & -1.43769600 & 0.43069900 \\ \mathrm{C} & 3.84128900 & 0.60455600 & -0.27871300 \\ \mathrm{C} & 6.07009200 & -0.17719900 & 0.02563300 \\ \mathrm{C} & 5.22400400 & 0.85733100 & -0.33490300 \\ \mathrm{~N} & 2.82204300 & 1.47634500 & -0.57727100 \\ \mathrm{~N} & 1.72642100 & 0.79262400 & -0.38503500 \\ \mathrm{~N} & 0.51006300 & 1.44444200 & -0.55983200 \\ \mathrm{H} & 3.80517500 & -2.65414200 & 0.79181400 \\ \mathrm{H} & 6.26427500 & -2.22222000 & 0.70175900 \\ \mathrm{H} & 7.14546800 & -0.02537400 & -0.00108300 \\ \mathrm{H} & 5.59814100 & 1.82718900 & -0.64727000 \\ \mathrm{H} & -0.26042800 & 0.78378800 & -0.41569100 \\ \mathrm{H} & 0.47985200 & 1.80090300 & -1.51495600\end{array}$

20b

Point Group: $\mathrm{C} 1$

B3LYP/6-31G* = -945.200687 au

B3LYP/6-31G* Zero Point Corrected Energy = -944.944569 au 


\begin{tabular}{lrrr} 
NIMAG = 1 & & \\
\multicolumn{4}{c}{} \\
B3LYP/6-31G* Optimized Cartesian Coordinates \\
$\mathrm{C}$ & -0.74886900 & 1.47760000 & 0.37727200 \\
$\mathrm{C}$ & -2.03380600 & 1.01716400 & 0.14399300 \\
$\mathrm{C}$ & -2.65639900 & -0.18570400 & -0.32757700 \\
$\mathrm{C}$ & -2.17740600 & -1.40619400 & -0.82556300 \\
$\mathrm{C}$ & -3.10903200 & -2.37649200 & -1.16849400 \\
$\mathrm{C}$ & -4.05215500 & 0.03479500 & -0.18613700 \\
$\mathrm{C}$ & -4.49941700 & -2.15268100 & -1.02294100 \\
$\mathrm{C}$ & -4.98936300 & -0.95263300 & -0.53288900 \\
$\mathrm{~N}$ & -4.31109700 & 1.29522300 & 0.30650900 \\
$\mathrm{~N}$ & -3.12986800 & 1.81888700 & 0.48593600 \\
$\mathrm{~N}$ & -3.00259300 & 3.13304100 & 0.90275100 \\
$\mathrm{H}$ & -1.11113900 & -1.57653000 & -0.94389600 \\
$\mathrm{H}$ & -2.76840100 & -3.33042000 & -1.56199400 \\
$\mathrm{H}$ & -5.19301200 & -2.93963700 & -1.30534300 \\
$\mathrm{H}$ & -6.05314900 & -0.76908600 & -0.41897500 \\
$\mathrm{H}$ & -2.00648300 & 3.24082300 & 1.12200900 \\
$\mathrm{H}$ & -3.58326300 & 3.25997300 & 1.72973100 \\
$\mathrm{C}$ & 0.43768800 & 0.96215900 & 0.17085400 \\
$\mathrm{C}$ & 1.61063100 & 0.49016200 & 0.04040700 \\
$\mathrm{C}$ & 2.98761100 & 0.49705000 & -0.22641200 \\
$\mathrm{C}$ & 3.61181200 & 1.53881300 & -0.95738300 \\
$\mathrm{C}$ & 4.95502700 & 1.45697000 & -1.28737600 \\
$\mathrm{C}$ & 3.79400300 & -0.60469900 & 0.19290500 \\
$\mathrm{C}$ & 5.73388800 & 0.36046500 & -0.87402900 \\
$\mathrm{C}$ & 5.15456100 & -0.66256600 & -0.13805500 \\
$\mathrm{~N}$ & 3.20760000 & -1.64475300 & 0.90326500 \\
$\mathrm{~N}$ & 2.09499300 & -1.29781800 & 1.39416600 \\
$\mathrm{~N}$ & 1.38304900 & -2.30658400 & 1.94966100 \\
$\mathrm{H}$ & 3.01315200 & 2.38631800 & -1.27586700 \\
$\mathrm{H}$ & 5.41116700 & 2.25481800 & -1.86715100 \\
$\mathrm{H}$ & 6.78921500 & 0.31781900 & -1.12685100 \\
$\mathrm{H}$ & 5.73137100 & -1.51835600 & 0.19886100 \\
$\mathrm{H}$ & 0.40737700 & -2.05164600 & 2.03906600 \\
$\mathrm{H}$ & 1.55593000 & -3.22529700 & 1.54294600
\end{tabular}

\footnotetext{
20c

Point Group: C1

B3LYP/6-31G* $=-945.19266$ au

B3LYP/6-31G* Zero Point Corrected Energy $=-944.93646$ au NIMAG $=1$

B3LYP/6-31G* Optimized Cartesian Coordinates

$\begin{array}{lrrr}\text { C } & -0.48990400 & -1.19445800 & 0.36390300 \\ \text { C } & -1.73858600 & -1.22962800 & 0.31988500 \\ \text { C } & -3.09933000 & -0.94587400 & 0.07134700 \\ \text { C } & -4.03025200 & -1.99154200 & -0.13288700 \\ \text { C } & -5.34507400 & -1.71913900 & -0.48657400 \\ \text { C } & -3.55620000 & 0.39848800 & -0.05288300\end{array}$
}




$\begin{array}{lrrr}\mathrm{C} & -5.76978100 & -0.39192700 & -0.62129600 \\ \mathrm{C} & -4.87866300 & 0.65977200 & -0.40845200 \\ \mathrm{~N} & -2.63627600 & 1.46889400 & 0.09202500 \\ \mathrm{~N} & -2.27142300 & 1.65104100 & 1.28494300 \\ \mathrm{~N} & -1.35685500 & 2.62460000 & 1.42201100 \\ \mathrm{H} & -3.68458400 & -3.01450900 & -0.02262800 \\ \mathrm{H} & -6.04259900 & -2.53479500 & -0.65332300 \\ \mathrm{H} & -6.80114300 & -0.17521400 & -0.88593600 \\ \mathrm{H} & -5.19928200 & 1.69218900 & -0.50991300 \\ \mathrm{H} & -0.87522500 & 2.55145000 & 2.30862400 \\ \mathrm{H} & -0.76289700 & 2.79234900 & 0.60699100 \\ \mathrm{C} & 0.83920100 & -1.34641400 & 0.49981000 \\ \mathrm{C} & 1.83313200 & -0.47943000 & 0.08187400 \\ \mathrm{C} & 3.26095500 & -0.61338900 & 0.14791800 \\ \mathrm{C} & 4.11089300 & -1.58816900 & 0.68739500 \\ \mathrm{C} & 5.47871500 & -1.39995600 & 0.55093700 \\ \mathrm{C} & 3.79843200 & 0.51664700 & -0.51624900 \\ \mathrm{C} & 6.01055100 & -0.26659800 & -0.11270100 \\ \mathrm{C} & 5.18478800 & 0.70353700 & -0.65581400 \\ \mathrm{~N} & 2.79508900 & 1.34349300 & -0.96769500 \\ \mathrm{~N} & 1.68883500 & 0.75047600 & -0.61402500 \\ \mathrm{~N} & 0.48439100 & 1.40142000 & -0.85500200 \\ \mathrm{H} & 3.69688500 & -2.45631000 & 1.19068000 \\ \mathrm{H} & 6.16369900 & -2.13780700 & 0.95968400 \\ \mathrm{H} & 7.08858100 & -0.16121500 & -0.19546000 \\ \mathrm{H} & 5.57840200 & 1.57578600 & -1.16800900 \\ \mathrm{H} & -0.27303600 & 0.71766800 & -0.79204100 \\ \mathrm{H} & 0.53160100 & 1.79942100 & -1.79174700\end{array}$

21

Point Group: C1

B3LYP/6-31G* $=-945.285052 \mathrm{au}$

B3LYP/6-31G* Zero Point Corrected Energy = -945.024474 au $\mathrm{NIMAG}=0$

B3LYP/6-31G* Optimized Cartesian Coordinates

$\begin{array}{lrrr}\mathrm{C} & 0.57848800 & -0.19016000 & 0.00000000 \\ \mathrm{C} & 1.93266700 & -0.54522600 & 0.00000000 \\ \mathrm{C} & 3.09933600 & 0.25147900 & 0.00000000 \\ \mathrm{C} & 3.35033900 & 1.64101100 & -0.00014500 \\ \mathrm{C} & 4.66446800 & 2.05663900 & -0.00019300 \\ \mathrm{C} & 4.17855300 & -0.69035300 & 0.00000000 \\ \mathrm{C} & 5.74083300 & 1.12066900 & -0.00016300 \\ \mathrm{C} & 5.52167700 & -0.23862700 & 0.00000000 \\ \mathrm{~N} & 3.72934300 & -1.96637700 & 0.00000000 \\ \mathrm{~N} & 2.39278600 & -1.83571500 & 0.00020800 \\ \mathrm{~N} & 1.55426000 & -2.95543700 & 0.00045900 \\ \mathrm{H} & 2.52768800 & 2.35033100 & -0.00017900 \\ \mathrm{H} & 4.89362600 & 3.11867400 & -0.00026700 \\ \mathrm{H} & 6.75999300 & 1.49803100 & -0.00022000 \\ \mathrm{H} & 6.33874900 & -0.95326900 & 0.00000000\end{array}$




$\begin{array}{lrrr}\mathrm{H} & 1.80519100 & -3.51105600 & -0.81802200 \\ \mathrm{H} & 1.80588400 & -3.51117200 & 0.81863800 \\ \mathrm{C} & -0.57848200 & 0.19054400 & 0.00000000 \\ \mathrm{C} & -1.93269900 & 0.54546600 & 0.00000000 \\ \mathrm{C} & -3.09924200 & -0.25142900 & 0.00000000 \\ \mathrm{C} & -3.35001400 & -1.64100100 & -0.00013500 \\ \mathrm{C} & -4.66407600 & -2.05684300 & -0.00018100 \\ \mathrm{C} & -4.17860700 & 0.69023300 & 0.00000000 \\ \mathrm{C} & -5.74059100 & -1.12104500 & -0.00016600 \\ \mathrm{C} & -5.52165800 & 0.23828600 & -0.00010200 \\ \mathrm{~N} & -3.72960000 & 1.96632800 & 0.00000000 \\ \mathrm{~N} & -2.39301700 & 1.83587500 & 0.00014400 \\ \mathrm{~N} & -1.55465800 & 2.95572000 & 0.00037300 \\ \mathrm{H} & -2.52724000 & -2.35017900 & -0.00012900 \\ \mathrm{H} & -4.89306500 & -3.11891400 & -0.00023200 \\ \mathrm{H} & -6.75969000 & -1.49857200 & -0.00022200 \\ \mathrm{H} & -6.33884900 & 0.95279200 & -0.00010200 \\ \mathrm{H} & -1.80562800 & 3.51096300 & 0.81909800 \\ \mathrm{H} & -1.80641600 & 3.51175700 & -0.81756000\end{array}$

21c

Point Group: C1

B3LYP/6-31G* $=-945.283778 \mathrm{au}$

B3LYP/6-31G* Zero Point Corrected Energy = -945.02340 au NIMAG $=0$

B3LYP/6-31G* Optimized Cartesian Coordinates

$\begin{array}{lrrr}\mathrm{C} & -0.60860000 & 0.61896200 & 0.02740600 \\ \mathrm{C} & -2.00808400 & 0.56768400 & 0.06948700 \\ \mathrm{C} & -2.90514500 & -0.51593500 & -0.07195500 \\ \mathrm{C} & -2.76093000 & -1.90201900 & -0.29794600 \\ \mathrm{C} & -3.90569000 & -2.66662300 & -0.37101300 \\ \mathrm{C} & -4.20411100 & 0.06997800 & 0.07158200 \\ \mathrm{C} & -5.19910400 & -2.08474700 & -0.22502200 \\ \mathrm{C} & -5.36669500 & -0.73572200 & -0.00511900 \\ \mathrm{~N} & -4.12988600 & 1.40662300 & 0.26992800 \\ \mathrm{~N} & -2.81244700 & 1.65891900 & 0.26666400 \\ \mathrm{~N} & -2.32610000 & 2.95461700 & 0.46871400 \\ \mathrm{H} & -1.77596900 & -2.34591500 & -0.41038100 \\ \mathrm{H} & -3.82822800 & -3.73642000 & -0.54384900 \\ \mathrm{H} & -6.07183700 & -2.72892200 & -0.29080000 \\ \mathrm{H} & -6.34952400 & -0.28845200 & 0.10590500 \\ \mathrm{H} & -2.50694800 & 3.48716200 & -0.38372800 \\ \mathrm{H} & -2.90926000 & 3.36020200 & 1.20025900 \\ \mathrm{C} & 0.60858200 & 0.61894100 & -0.02677900 \\ \mathrm{C} & 2.00805500 & 0.56768800 & -0.06920600 \\ \mathrm{C} & 2.90518600 & -0.51589100 & 0.07212500 \\ \mathrm{C} & 2.76106900 & -1.90195500 & 0.29829200 \\ \mathrm{C} & 3.90587100 & -2.66652400 & 0.37111100 \\ \mathrm{C} & 4.20409700 & 0.07004000 & -0.07183000 \\ \mathrm{C} & 5.19922700 & -2.08463000 & 0.22470000\end{array}$




$\begin{array}{lrrr}\mathrm{C} & 5.36672100 & -0.73562200 & 0.00461800 \\ \mathrm{~N} & 4.12977900 & 1.40667100 & -0.27025400 \\ \mathrm{~N} & 2.81233800 & 1.65892900 & -0.26666800 \\ \mathrm{~N} & 2.32591700 & 2.95459800 & -0.46873700 \\ \mathrm{H} & 1.77615100 & -2.34586700 & 0.41104100 \\ \mathrm{H} & 3.82848300 & -3.73630600 & 0.54407100 \\ \mathrm{H} & 6.07199600 & -2.72877600 & 0.29029800 \\ \mathrm{H} & 6.34950800 & -0.28833600 & -0.10672200 \\ \mathrm{H} & 2.90922700 & 3.36026800 & -1.20011300 \\ \mathrm{H} & 2.50649600 & 3.48711100 & 0.38378400\end{array}$

\section{2a}

Point Group: CI

B3LYP/6-31G* $=-869.056037 \mathrm{au}$

B3LYP/6-31G* Zero Point Corrected Energy $=-868.81123 \mathrm{au}$ $\mathrm{NIMAG}=0$

$\begin{array}{lrrr}\text { B3LYP/6-31G* Optimized Cartesian Coordinates } \\ \text { C } & 1.91424200 & -0.67189100 & 0.01167200 \\ \mathrm{C} & 2.13191500 & -2.06454400 & -0.03381900 \\ \mathrm{C} & 3.41870600 & -2.58799200 & -0.02920000 \\ \mathrm{C} & 3.03757900 & 0.19263900 & 0.05201900 \\ \mathrm{C} & 4.52143100 & -1.72673600 & 0.02377900 \\ \mathrm{C} & 4.33101600 & -0.35026700 & 0.06724200 \\ \mathrm{~N} & 2.76092100 & 1.57859300 & 0.11029200 \\ \mathrm{~N} & 3.74403100 & 2.31535600 & -0.16582700 \\ \mathrm{~N} & 3.47337800 & 3.62662100 & 0.01577300 \\ \mathrm{H} & 1.26695400 & -2.71915600 & -0.07199100 \\ \mathrm{H} & 3.56590800 & -3.66404300 & -0.05898700 \\ \mathrm{H} & 5.52977300 & -2.13165900 & 0.03908600 \\ \mathrm{H} & 5.17638000 & 0.32696300 & 0.11887000 \\ \mathrm{H} & 4.04722400 & 4.21667700 & -0.57285300 \\ \mathrm{H} & 2.47951300 & 3.85745900 & 0.05704400 \\ \mathrm{C} & 0.58327400 & -0.17186100 & 0.00481500 \\ \mathrm{C} & -0.58327400 & 0.17186100 & -0.00481500 \\ \mathrm{C} & -1.91424200 & 0.67189100 & -0.01167200 \\ \mathrm{C} & -2.13191500 & 2.06454400 & 0.03381900 \\ \mathrm{C} & -3.41870600 & 2.58799200 & 0.02920000 \\ \mathrm{C} & -3.03757900 & -0.19263900 & -0.05201900 \\ \mathrm{C} & -4.52143100 & 1.72673600 & -0.02377900 \\ \mathrm{C} & -4.33101600 & 0.35026700 & -0.06724200 \\ \mathrm{~N} & -2.76092100 & -1.57859300 & -0.11029200 \\ \mathrm{~N} & -3.74403100 & -2.31535600 & 0.16582700 \\ \mathrm{~N} & -3.47337800 & -3.62662100 & -0.01577300 \\ \mathrm{H} & -1.26695400 & 2.71915600 & 0.07199100 \\ \mathrm{H} & -3.56590800 & 3.66404300 & 0.05898700 \\ \mathrm{H} & -5.52977300 & 2.13165900 & -0.03908600 \\ \mathrm{H} & -5.17638000 & -0.32696300 & -0.11887000 \\ \mathrm{H} & -4.04722400 & -4.21667700 & 0.57285300 \\ \mathrm{H} & -2.47951300 & -3.85745900 & -0.05704400 \\ & & & \\ & & & \end{array}$




$$
\begin{aligned}
& \text { 22b } \\
& \text { Point Group: CI } \\
& \text { B3LYP/6-31G* }=-869.050207 \mathrm{au} \\
& \text { B3LYP/6-31G* Zero Point Corrected Energy }=-868.80556 \mathrm{au} \\
& \mathrm{NIMAG}=0 \\
& \text { B3LYP/6-31G* Optimized Cartesian Coordinates } \\
& \begin{array}{llll}
\text { C } & -1.98965100 & 0.37922000 & 0.05209300
\end{array} \\
& \begin{array}{llll}
\text { C } & -2.35908200 & 1.73694300 & 0.16499900
\end{array} \\
& \begin{array}{llll}
\text { C } & -3.69173800 & 2.13160000 & 0.14571200
\end{array} \\
& \begin{array}{llll}
\text { C } & -3.02275200 & -0.58960000 & -0.07876600
\end{array} \\
& \begin{array}{lllll}
\text { C } & & -4.69858800 & 1.17391100 & -0.00998800
\end{array} \\
& \begin{array}{llll}
\text { C } & -4.35798800 & -0.16887000 & -0.13515700
\end{array} \\
& \begin{array}{llll}
\mathrm{N} & -2.83078000 & -1.98243900 & -0.23140000
\end{array} \\
& \mathrm{~N} \quad-1.82428700 \quad-2.44851100 \quad 0.36286500 \\
& \begin{array}{llll}
\mathrm{N} & -1.61992700 & -3.75640900 & 0.08480700
\end{array} \\
& \begin{array}{llll}
\mathrm{H} & -1.56595600 & 2.47208700 & 0.26254400
\end{array} \\
& \begin{array}{llll}
\mathrm{H} & -3.94477700 & 3.18422100 & 0.23766900
\end{array} \\
& \mathrm{H} \quad-5.74251600 \quad 1.47332200 \quad-0.03988500 \\
& \mathrm{H} \quad-5.12011400 \quad-0.93081400 \quad-0.26740400 \\
& \mathrm{H} \quad-1.14402000 \quad-4.22988300 \quad 0.84217900 \\
& \mathrm{H} \quad-2.42545100 \quad-4.24188500 \quad-0.31462800 \\
& \begin{array}{llll}
\text { C } & -0.60457800 & 0.06362000 & 0.02300700
\end{array} \\
& \text { C } \quad 0.60457800 \quad-0.06362000 \quad-0.02300700 \\
& \begin{array}{llll}
\text { C } & 1.98965100 & -0.37922000 & -0.05209300
\end{array} \\
& \text { C } \quad 2.35908200 \quad-1.73694300 \quad-0.16499900 \\
& \begin{array}{llll}
\text { C } & 3.69173800 & -2.13160000 & -0.14571200
\end{array} \\
& \begin{array}{llll}
\mathrm{C} & 3.02275200 & 0.58960000 & 0.07876600
\end{array} \\
& \begin{array}{llll}
\text { C } & 4.69858800 & -1.17391100 & 0.00998800
\end{array} \\
& \begin{array}{llll}
\mathrm{C} & 4.35798800 & 0.16887000 & 0.13515700
\end{array} \\
& \begin{array}{llll}
\mathrm{N} & 2.83078000 & 1.98243900 & 0.23140000
\end{array} \\
& \mathrm{~N} \quad \begin{array}{llll}
1.82428700 & 2.44851100 & -0.36286500
\end{array} \\
& \mathrm{~N} \quad 1.61992700 \quad 3.75640900 \quad-0.08480700 \\
& \mathrm{H} \quad \begin{array}{llll}
1.56595600 & -2.47208700 & -0.26254400
\end{array} \\
& \mathrm{H} \quad 3.94477700 \quad-3.18422100 \quad-0.23766900 \\
& \mathrm{H} \quad \begin{array}{llll}
\mathrm{H} & 5.74251600 & -1.47332200 & 0.03988500
\end{array} \\
& \begin{array}{llll}
\mathrm{H} & 5.12011400 & 0.93081400 & 0.26740400
\end{array} \\
& \mathrm{H} \quad 1.14402000 \quad 4.22988300 \quad-0.84217900 \\
& \begin{array}{llll}
\mathrm{H} & 2.42545100 & 4.24188500 & 0.31462800
\end{array}
\end{aligned}
$$

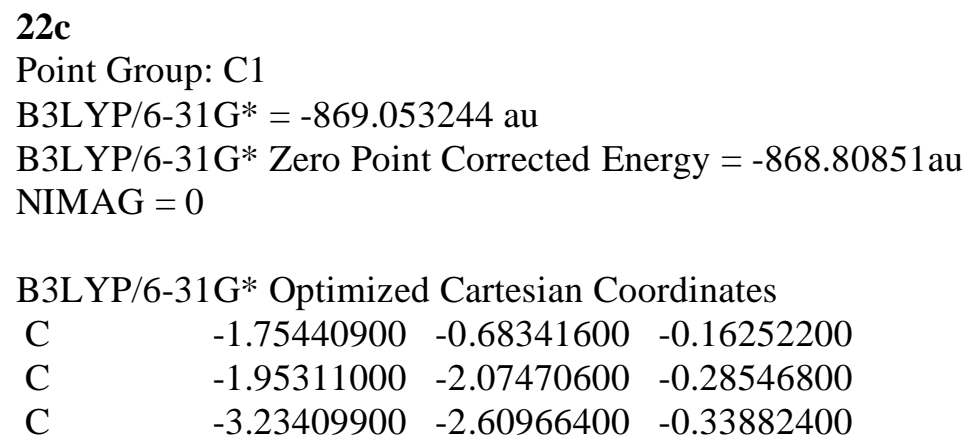




$\begin{array}{lrrr}\mathrm{C} & -2.88774500 & 0.16424200 & -0.08110200 \\ \mathrm{C} & -4.34791500 & -1.76359300 & -0.26897600 \\ \mathrm{C} & -4.17504100 & -0.38982200 & -0.14347000 \\ \mathrm{~N} & -2.62578900 & 1.55001500 & 0.02710100 \\ \mathrm{~N} & -3.61125100 & 2.23717200 & 0.40373500 \\ \mathrm{~N} & -3.35472800 & 3.56413400 & 0.37881200 \\ \mathrm{H} & -1.07945800 & -2.71674800 & -0.34174300 \\ \mathrm{H} & -3.36860900 & -3.68282200 & -0.44283600 \\ \mathrm{H} & -5.35141700 & -2.17749000 & -0.32087400 \\ \mathrm{H} & -5.02914400 & 0.27685900 & -0.09872600 \\ \mathrm{H} & -3.92382500 & 4.07135400 & 1.04432200 \\ \mathrm{H} & -2.36331600 & 3.80775200 & 0.35388700 \\ \mathrm{C} & -0.42888600 & -0.17286800 & -0.11698000 \\ \mathrm{C} & 0.74465200 & 0.14576000 & -0.08032700 \\ \mathrm{C} & 2.07735400 & 0.63970100 & -0.07838400 \\ \mathrm{C} & 2.27395500 & 2.03515700 & -0.15488300 \\ \mathrm{C} & 3.54701900 & 2.58945900 & -0.21746700 \\ \mathrm{C} & 3.22653400 & -0.19902700 & -0.06148900 \\ \mathrm{C} & 4.66885600 & 1.75490400 & -0.22377800 \\ \mathrm{C} & 4.50059600 & 0.37583400 & -0.16053200 \\ \mathrm{~N} & 3.21267500 & -1.61190300 & -0.02149300 \\ \mathrm{~N} & 2.25221100 & -2.11626000 & 0.61649600 \\ \mathrm{~N} & 2.21645300 & -3.46424400 & 0.51591300 \\ \mathrm{H} & 1.39438300 & 2.67117200 & -0.17483800 \\ \mathrm{H} & 3.66434800 & 3.66794700 & -0.27636800 \\ \mathrm{H} & 5.66805100 & 2.17688700 & -0.28522500 \\ \mathrm{H} & 5.35446200 & -0.29465900 & -0.17557600 \\ \mathrm{H} & 1.77464600 & -3.88875000 & 1.32139200 \\ \mathrm{H} & 3.08631500 & -3.89565500 & 0.19785200\end{array}$

\section{3b}

Point Group: $\mathrm{C} 1$

B3LYP/6-31G* $=-869.014254$ au

B3LYP/6-31G* Zero Point Corrected Energy $=-868.77067 \mathrm{au}$ NIMAG $=1$

B3LYP/6-31G* Optimized Cartesian Coordinates

$\begin{array}{lrrr}\mathrm{C} & 2.12159700 & 0.16153600 & -0.38058800 \\ \mathrm{C} & 2.70479100 & 1.16369600 & -1.16771200 \\ \mathrm{C} & 4.09307100 & 1.26101300 & -1.22117600 \\ \mathrm{C} & 2.96192500 & -0.71980600 & 0.33591000 \\ \mathrm{C} & 4.91604000 & 0.37458000 & -0.50502600 \\ \mathrm{C} & 4.35320600 & -0.62628200 & 0.28143800 \\ \mathrm{~N} & 2.28006700 & -1.68280100 & 1.09982900 \\ \mathrm{~N} & 1.04838900 & -1.48240300 & 0.93054700 \\ \mathrm{~N} & 0.14278200 & -2.21203800 & 1.62232400 \\ \mathrm{H} & 2.06747200 & 1.84628300 & -1.72097200 \\ \mathrm{H} & 4.54838200 & 2.03778700 & -1.82963700 \\ \mathrm{H} & 5.99596300 & 0.47273700 & -0.56460800 \\ \mathrm{H} & 4.96367800 & -1.32319500 & 0.84745000 \\ \mathrm{H} & -0.74891400 & -2.21609400 & 1.13565600\end{array}$




$\begin{array}{lrrr}\mathrm{H} & 0.49558600 & -3.12808900 & 1.89324700 \\ \mathrm{C} & 0.72552800 & -0.10872000 & -0.20785100 \\ \mathrm{C} & -0.46268900 & 0.25187600 & -0.54817500 \\ \mathrm{C} & -1.79523400 & -0.22296700 & -0.50067600 \\ \mathrm{C} & -2.12095100 & -1.51148600 & -1.00745100 \\ \mathrm{C} & -3.43326200 & -1.96379400 & -1.05436300 \\ \mathrm{C} & -2.87906100 & 0.58736600 & -0.04106600 \\ \mathrm{C} & -4.48220100 & -1.15683100 & -0.58914300 \\ \mathrm{C} & -4.19658800 & 0.10998200 & -0.09781900 \\ \mathrm{~N} & -2.69546200 & 1.88915200 & 0.43105000 \\ \mathrm{~N} & -1.50247700 & 2.14943400 & 0.75444300 \\ \mathrm{~N} & -1.29883300 & 3.47078300 & 0.99735200 \\ \mathrm{H} & -1.31745300 & -2.12665100 & -1.40562100 \\ \mathrm{H} & -3.64412800 & -2.94941600 & -1.46206800 \\ \mathrm{H} & -5.50759200 & -1.51281400 & -0.62368300 \\ \mathrm{H} & -4.98486000 & 0.76875800 & 0.25467800 \\ \mathrm{H} & -0.56843700 & 3.60714600 & 1.68579100 \\ \mathrm{H} & -2.15799600 & 3.99767800 & 1.16312900\end{array}$

\section{3c}

Point Group: C1

B3LYP/6-31G* $=-869.010832 \mathrm{au}$

B3LYP/6-31G* Zero Point Corrected Energy $=-868.76732 \mathrm{au}$

NIMAG $=1$

B3LYP/6-31G* Optimized Cartesian Coordinates

$\begin{array}{lrrr}\mathrm{C} & 2.25897800 & -0.33826400 & -0.43150400 \\ \mathrm{C} & 2.93960500 & -0.60021900 & -1.62407900 \\ \mathrm{C} & 4.32572800 & -0.45557100 & -1.65243900 \\ \mathrm{C} & 2.98925500 & 0.06644100 & 0.70644400 \\ \mathrm{C} & 5.04074000 & -0.04907200 & -0.51219400 \\ \mathrm{C} & 4.37727100 & 0.21269500 & 0.68337900 \\ \mathrm{~N} & 2.19094000 & 0.26945600 & 1.84256800 \\ \mathrm{~N} & 0.98938300 & 0.17440400 & 1.45429900 \\ \mathrm{~N} & -0.02175900 & 0.26772100 & 2.34525700 \\ \mathrm{H} & 2.38461200 & -0.92100700 & -2.50018500 \\ \mathrm{H} & 4.86506400 & -0.66696500 & -2.57178000 \\ \mathrm{H} & 6.12026100 & 0.05861600 & -0.56447100 \\ \mathrm{H} & 4.90695400 & 0.52656800 & 1.57751300 \\ \mathrm{H} & -0.88307800 & -0.05891300 & 1.91636800 \\ \mathrm{H} & 0.19665900 & -0.15648800 & 3.24491200 \\ \mathrm{C} & 0.84862900 & -0.44547000 & -0.15003000 \\ \mathrm{C} & -0.25225300 & -0.88168400 & -0.66487100 \\ \mathrm{C} & -1.64441400 & -0.98140200 & -0.38572200 \\ \mathrm{C} & -2.23683300 & -2.26715100 & -0.26802400 \\ \mathrm{C} & -3.59138600 & -2.41981400 & -0.01511400 \\ \mathrm{C} & -2.50838000 & 0.15166800 & -0.28539300 \\ \mathrm{C} & -4.42674500 & -1.29572800 & 0.07410900 \\ \mathrm{C} & -3.88502800 & -0.02482200 & -0.07080500 \\ \mathrm{~N} & -1.88414400 & 1.41626800 & -0.37366000 \\ \mathrm{~N} & -2.65637100 & 2.37140500 & -0.65101100\end{array}$




$\begin{array}{lrrr}\mathrm{N} & -2.03506900 & 3.57565700 & -0.57383700 \\ \mathrm{H} & -1.59594600 & -3.13688300 & -0.37730200 \\ \mathrm{H} & -4.00783400 & -3.41776000 & 0.09591000 \\ \mathrm{H} & -5.49117700 & -1.41565700 & 0.25556000 \\ \mathrm{H} & -4.51013400 & 0.85881400 & 0.00293100 \\ \mathrm{H} & -2.46178600 & 4.25107800 & -1.19589500 \\ \mathrm{H} & -1.01545800 & 3.52458700 & -0.61142400\end{array}$

\begin{tabular}{lrrr} 
24c & \multicolumn{4}{l}{} \\
Point Group: C1 & \\
B3LYP/6-31G* $=-869.017468$ au \\
B3LYP/6-31G* Zero Point Corrected Energy $=-868.7721$ \\
NIMAG = 0 \\
\multicolumn{4}{c}{} \\
B3LYP/6-31G* Optimized Cartesian Coordinates \\
$\mathrm{C}$ & 2.25526500 & -0.20231200 & -0.45733500 \\
$\mathrm{C}$ & 2.95412600 & -0.15822500 & -1.66676100 \\
$\mathrm{C}$ & 4.33461500 & 0.00006800 & -1.61263400 \\
$\mathrm{C}$ & 2.94771700 & -0.09479300 & 0.76759400 \\
$\mathrm{C}$ & 5.02039800 & 0.11132800 & -0.38165600 \\
$\mathrm{C}$ & 4.33967600 & 0.06323900 & 0.82668700 \\
$\mathrm{~N}$ & 2.07190700 & -0.17500800 & 1.83786900 \\
$\mathrm{~N}$ & 0.89967600 & -0.30852900 & 1.31326800 \\
$\mathrm{~N}$ & -0.19926900 & -0.33693100 & 2.13639900 \\
$\mathrm{H}$ & 2.42063000 & -0.24927300 & -2.60769600 \\
$\mathrm{H}$ & 4.90464800 & 0.03738400 & -2.53698800 \\
$\mathrm{H}$ & 6.09948300 & 0.23540800 & -0.38536000 \\
$\mathrm{H}$ & 4.85047400 & 0.14586100 & 1.78076600 \\
$\mathrm{H}$ & -0.98359600 & -0.74622500 & 1.63144100 \\
$\mathrm{H}$ & 0.03765000 & -0.84492900 & 2.98531800 \\
$\mathrm{C}$ & 0.85607900 & -0.37018600 & -0.13434800 \\
$\mathrm{C}$ & -0.20558600 & -0.62592300 & -0.94299100 \\
$\mathrm{C}$ & -1.57190600 & -0.84519100 & -0.56877100 \\
$\mathrm{C}$ & -2.10605200 & -2.16280400 & -0.60646200 \\
$\mathrm{C}$ & -3.44144900 & -2.40570200 & -0.31833700 \\
$\mathrm{C}$ & -2.47506300 & 0.21927200 & -0.27312400 \\
$\mathrm{C}$ & -4.31661200 & -1.34641000 & -0.03350200 \\
$\mathrm{C}$ & -3.83042300 & -0.04516700 & -0.02191600 \\
$\mathrm{~N}$ & -1.88489800 & 1.50393500 & -0.24947700 \\
$\mathrm{~N}$ & -2.69355500 & 2.46707300 & -0.21394800 \\
$\mathrm{~N}$ & -2.06648300 & 3.66327200 & -0.06757900 \\
$\mathrm{H}$ & -1.44396700 & -2.98355600 & -0.86697000 \\
$\mathrm{H}$ & -3.81081300 & -3.42817400 & -0.33095000 \\
$\mathrm{H}$ & -5.36495200 & -1.53962100 & 0.17461500 \\
$\mathrm{H}$ & -4.48368400 & 0.79219700 & 0.19944600 \\
$\mathrm{H}$ & -2.60651500 & 4.41329500 & -0.48146200 \\
$\mathrm{H}$ & -1.07571000 & 3.64777700 & -0.31654100 \\
& & & \\
& &
\end{tabular}

25c

Point Group: C1 


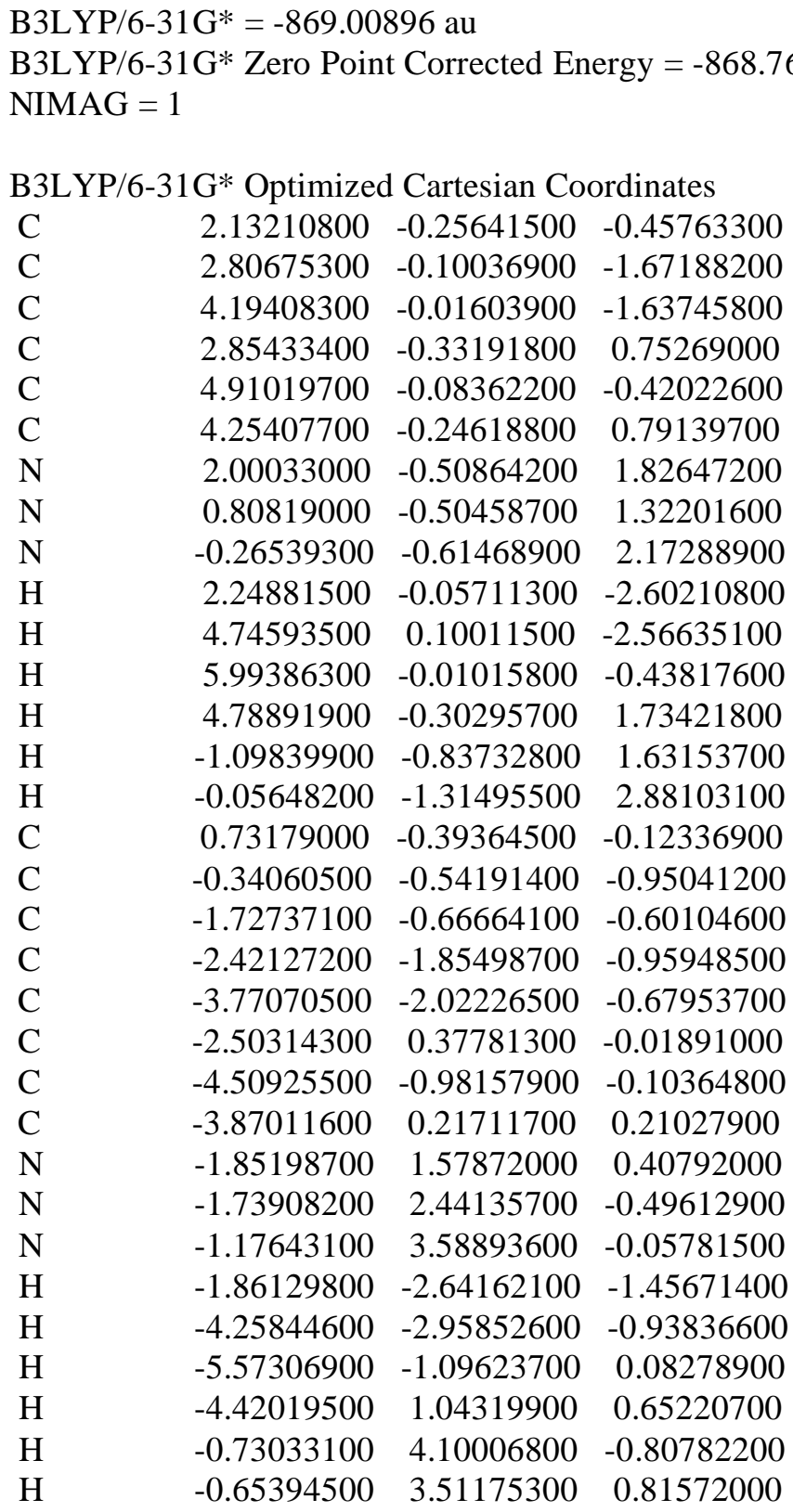

\section{6}

Point Group: $\mathrm{C} 1$

B3LYP/6-31G* $=-869.124392 \mathrm{au}$

B3LYP/6-31G* Zero Point Corrected Energy $=-868.87403 \mathrm{au}$ NIMAG $=0$

B3LYP/6-31G* Optimized Cartesian Coordinates

$\begin{array}{lrrr}\mathrm{C} & -0.01699800 & 0.71545700 & -0.26285300 \\ \mathrm{C} & 0.89054700 & 1.69446100 & 0.18819900 \\ \mathrm{C} & 2.15037900 & 1.67212100 & 0.83299900 \\ \mathrm{C} & 2.73866600 & 2.87484000 & 1.15281900 \\ \mathrm{C} & 0.25317900 & 2.94516400 & -0.10377600 \\ \mathrm{C} & 2.10711400 & 4.11804200 & 0.84958300\end{array}$




$\begin{array}{lrrr}\mathrm{C} & 0.87927800 & 4.17214500 & 0.23183000 \\ \mathrm{~N} & -0.94851500 & 2.76624600 & -0.69383600 \\ \mathrm{~N} & -1.07043900 & 1.42978100 & -0.76345200 \\ \mathrm{~N} & -2.19710700 & 0.85433000 & -1.36527000 \\ \mathrm{H} & 2.63754300 & 0.73113100 & 1.06653700 \\ \mathrm{H} & 3.70580500 & 2.88569900 & 1.64796700 \\ \mathrm{H} & 2.61377100 & 5.04097200 & 1.11916700 \\ \mathrm{H} & 0.39025800 & 5.11391100 & 0.00287400 \\ \mathrm{H} & -3.00824500 & 1.30852300 & -0.94533600 \\ \mathrm{H} & -2.18528000 & 1.13846000 & -2.34608200 \\ \mathrm{C} & 0.00936500 & -0.73602200 & -0.20691900 \\ \mathrm{C} & -0.90869200 & -1.67891300 & 0.29675600 \\ \mathrm{C} & -2.18328700 & -1.60907900 & 0.90831400 \\ \mathrm{C} & -2.77862300 & -2.78449800 & 1.30686800 \\ \mathrm{C} & -0.26486600 & -2.94732900 & 0.11675600 \\ \mathrm{C} & -2.14001000 & -4.04623900 & 1.11556300 \\ \mathrm{C} & -0.89840400 & -4.14578300 & 0.53199300 \\ \mathrm{~N} & 0.95024500 & -2.81237700 & -0.45731600 \\ \mathrm{~N} & 1.07399600 & -1.48502000 & -0.62667300 \\ \mathrm{~N} & 2.21394100 & -0.95578700 & -1.24554900 \\ \mathrm{H} & -2.67595900 & -0.65372800 & 1.05594200 \\ \mathrm{H} & -3.75708500 & -2.75881400 & 1.77867200 \\ \mathrm{H} & -2.65261600 & -4.94646400 & 1.44420500 \\ \mathrm{H} & -0.40413000 & -5.10155400 & 0.38820500 \\ \mathrm{H} & 2.22338100 & -1.31322400 & -2.20209700 \\ \mathrm{H} & 3.01533700 & -1.37603800 & -0.77468200\end{array}$

27b

Point Group: C2

B3LYP/6-31G* $=-1296.61773 \mathrm{au}$

B3LYP/6-31G* Zero Point Corrected Energy $=-1296.23711$ au NIMAG $=1$

B3LYP/6-31G* Optimized Cartesian Coordinates

$\begin{array}{lrrr}\mathrm{C} & -0.18856100 & 0.62551300 & -1.11425400 \\ \mathrm{C} & -0.41487800 & 1.87131300 & -1.00916600 \\ \mathrm{C} & -1.15065700 & 3.02753900 & -1.32705300 \\ \mathrm{C} & -2.25152500 & 3.04161500 & -2.21248200 \\ \mathrm{C} & -2.91143600 & 4.23247100 & -2.47614800 \\ \mathrm{C} & -0.75697400 & 4.25618400 & -0.72879900 \\ \mathrm{C} & -2.51022800 & 5.44288800 & -1.87471900 \\ \mathrm{C} & -1.43341900 & 5.45467700 & -1.00334800 \\ \mathrm{~N} & 0.33024400 & 4.22939300 & 0.12748900 \\ \mathrm{~N} & 0.75697400 & 3.04524800 & 0.27841300 \\ \mathrm{C} & 1.90900000 & 2.83445200 & 1.05913100 \\ \mathrm{H} & -2.56537800 & 2.11399400 & -2.68068500 \\ \mathrm{H} & -3.75459300 & 4.23002600 & -3.16188700 \\ \mathrm{H} & -3.04466700 & 6.36159800 & -2.09656500 \\ \mathrm{H} & -1.09328500 & 6.36640000 & -0.52183200 \\ \mathrm{C} & 0.18856100 & -0.62551300 & -1.11425400 \\ \mathrm{C} & 0.41487800 & -1.87131300 & -1.00916600\end{array}$




$\begin{array}{lrrr}\mathrm{C} & 1.15065700 & -3.02753900 & -1.32705300 \\ \mathrm{C} & 2.25152500 & -3.04161500 & -2.21248200 \\ \mathrm{C} & 2.91143600 & -4.23247100 & -2.47614800 \\ \mathrm{C} & 0.75697400 & -4.25618400 & -0.72879900 \\ \mathrm{C} & 2.51022800 & -5.44288800 & -1.87471900 \\ \mathrm{C} & 1.43341900 & -5.45467700 & -1.00334800 \\ \mathrm{~N} & -0.33024400 & -4.22939300 & 0.12748900 \\ \mathrm{~N} & -0.75697400 & -3.04524800 & 0.27841300 \\ \mathrm{C} & -1.90900000 & -2.83445200 & 1.05913100 \\ \mathrm{H} & 2.56537800 & -2.11399400 & -2.68068500 \\ \mathrm{H} & 3.75459300 & -4.23002600 & -3.16188700 \\ \mathrm{H} & 3.04466700 & -6.36159800 & -2.09656500 \\ \mathrm{H} & 1.09328500 & -6.36640000 & -0.52183200 \\ \mathrm{C} & -2.71900100 & -3.88735600 & 1.52131900 \\ \mathrm{C} & -2.24151300 & -1.50457900 & 1.36050700 \\ \mathrm{C} & -3.84494900 & -3.60103300 & 2.28420300 \\ \mathrm{C} & -3.37345300 & -1.23211100 & 2.12571900 \\ \mathrm{C} & -4.17685700 & -2.27566600 & 2.58959400 \\ \mathrm{C} & 2.24151300 & 1.50457900 & 1.36050700 \\ \mathrm{C} & 3.37345300 & 1.23211100 & 2.12571900 \\ \mathrm{C} & 4.17685700 & 2.27566600 & 2.58959400 \\ \mathrm{C} & 3.84494900 & 3.60103300 & 2.28420300 \\ \mathrm{C} & 2.71900100 & 3.88735600 & 1.52131900 \\ \mathrm{H} & -2.44922400 & -4.90733700 & 1.27208100 \\ \mathrm{H} & -1.60955600 & -0.70391600 & 0.99414200 \\ \mathrm{H} & -4.47167900 & -4.41362500 & 2.64203600 \\ \mathrm{H} & -3.62746700 & -0.20211900 & 2.35961100 \\ \mathrm{H} & -5.05999600 & -2.06027700 & 3.18476300 \\ \mathrm{H} & 1.60955600 & 0.70391600 & 0.99414200 \\ \mathrm{H} & 3.62746700 & 0.20211900 & 2.35961100 \\ \mathrm{H} & 5.05999600 & 2.06027700 & 3.18476300 \\ \mathrm{H} & 4.47167900 & 4.41362500 & 2.64203600 \\ \mathrm{H} & 2.44922400 & 4.90733700 & 1.27208100\end{array}$

\footnotetext{
27c

Point Group: C1

B3LYP/6-31G* $=-1296.61796$ au

B3LYP/6-31G* Zero Point Corrected Energy $=-1296.23675$ au $\mathrm{NIMAG}=1$

B3LYP/6-31G* Optimized Cartesian Coordinates

$\begin{array}{lrrr}\mathrm{C} & 0.21330600 & -1.24067300 & -1.10719300 \\ \mathrm{C} & -0.93182900 & -1.69611900 & -1.00165400 \\ \mathrm{C} & -2.19524300 & -2.30434500 & -0.87642200 \\ \mathrm{C} & -2.31408100 & -3.71290100 & -0.97647100 \\ \mathrm{C} & -3.55035500 & -4.32832700 & -0.85698600 \\ \mathrm{C} & -3.37560700 & -1.54252700 & -0.64878900 \\ \mathrm{C} & -4.71014400 & -3.56766800 & -0.63140700 \\ \mathrm{C} & -4.61819600 & -2.18844100 & -0.52872700 \\ \mathrm{~N} & -3.18640800 & -0.15139300 & -0.56172800 \\ \mathrm{~N} & -4.23025800 & 0.52547100 & -0.34023500\end{array}$
}




$\begin{array}{lrrr}\mathrm{H} & -1.41993000 & -4.30265000 & -1.15194000 \\ \mathrm{H} & -3.61832500 & -5.40992900 & -0.93980800 \\ \mathrm{H} & -5.67475300 & -4.05870600 & -0.53890800 \\ \mathrm{H} & -5.49431100 & -1.57335300 & -0.35578200 \\ \mathrm{C} & 1.44019200 & -0.74615900 & -1.29397900 \\ \mathrm{C} & 2.60809300 & -0.35495800 & -0.92861300 \\ \mathrm{C} & 3.84257100 & 0.10492600 & -1.47627400 \\ \mathrm{C} & 4.17291000 & 0.24128000 & -2.83345200 \\ \mathrm{C} & 5.43362600 & 0.71758300 & -3.17497200 \\ \mathrm{C} & 4.80228100 & 0.45617400 & -0.50093200 \\ \mathrm{C} & 6.38359600 & 1.06125800 & -2.19087000 \\ \mathrm{C} & 6.07362100 & 0.93593000 & -0.84461300 \\ \mathrm{~N} & 4.36967100 & 0.31120700 & 0.80794600 \\ \mathrm{~N} & 3.21286500 & -0.21609500 & 0.81367100 \\ \mathrm{H} & 3.44082100 & -0.02030800 & -3.59091300 \\ \mathrm{H} & 5.69259300 & 0.83038800 & -4.22422900 \\ \mathrm{H} & 7.36040400 & 1.42767900 & -2.49186400 \\ \mathrm{H} & 6.78106300 & 1.19345900 & -0.06255300 \\ \mathrm{C} & -4.01996900 & 1.92406000 & -0.26379500 \\ \mathrm{C} & -2.77593700 & 2.55804700 & -0.43524600 \\ \mathrm{C} & -5.16258500 & 2.69197700 & 0.00108000 \\ \mathrm{C} & -2.69188100 & 3.94193900 & -0.33856000 \\ \mathrm{H} & -1.90292200 & 1.94883700 & -0.64130500 \\ \mathrm{C} & -5.07030100 & 4.07926200 & 0.09731400 \\ \mathrm{H} & -6.10968900 & 2.17596600 & 0.12731700 \\ \mathrm{C} & -3.83495400 & 4.70712700 & -0.07226100 \\ \mathrm{H} & -1.73146500 & 4.43337700 & -0.47173100 \\ \mathrm{H} & -5.95941500 & 4.66910000 & 0.30306000 \\ \mathrm{H} & -3.75910900 & 5.78884200 & 0.00087900 \\ \mathrm{C} & 2.51768900 & -0.34936300 & 2.03475800 \\ \mathrm{C} & 2.88414700 & 0.39870800 & 3.16676500 \\ \mathrm{C} & 1.44110300 & -1.24578100 & 2.09276700 \\ \mathrm{C} & 2.17505600 & 0.23804000 & 4.35106300 \\ \mathrm{H} & 3.71404500 & 1.09251700 & 3.09569000 \\ \mathrm{C} & 0.73799500 & -1.39312000 & 3.28676200 \\ \mathrm{H} & 1.16401600 & -1.81722600 & 1.21620900 \\ \mathrm{C} & 1.10027400 & -0.65607400 & 4.41538000 \\ \mathrm{H} & 2.45508400 & 0.81681100 & 5.22685900 \\ \mathrm{H} & -0.09698800 & -2.08597200 & 3.33124100 \\ \mathrm{H} & 0.54552600 & -0.77230000 & 5.34216800\end{array}$

\footnotetext{
28c

Point Group: $\mathrm{C} 1$

B3LYP/6-31G* $=-1296.63976$ au

B3LYP/6-31G* Zero Point Corrected Energy $=-1296.25597 \mathrm{au}$ $\mathrm{NIMAG}=0$

B3LYP/6-31G* Optimized Cartesian Coordinates

$\begin{array}{lrrr}\mathrm{C} & 1.56811500 & 1.24243200 & 0.03324700 \\ \mathrm{C} & 2.10846700 & 0.03175700 & 0.43687900 \\ \mathrm{C} & 1.63400300 & -1.05388100 & 1.24292600\end{array}$
}




$\begin{array}{lrrr}\mathrm{C} & 0.41143700 & -1.33841500 & 1.87448900 \\ \mathrm{C} & 0.31799700 & -2.52472000 & 2.58600400 \\ \mathrm{C} & 2.72081900 & -1.95876000 & 1.34499900 \\ \mathrm{C} & 1.40966000 & -3.42741600 & 2.68531800 \\ \mathrm{C} & 2.61923900 & -3.16284100 & 2.07192200 \\ \mathrm{~N} & 3.80191100 & -1.50487200 & 0.64786500 \\ \mathrm{~N} & 3.43725900 & -0.34876200 & 0.12494200 \\ \mathrm{H} & -0.42562900 & -0.65166800 & 1.80084800 \\ \mathrm{H} & -0.61506800 & -2.77571700 & 3.08288000 \\ \mathrm{H} & 1.28465900 & -4.34263600 & 3.25697400 \\ \mathrm{H} & 3.46331900 & -3.84194200 & 2.13859400 \\ \mathrm{C} & 0.30062200 & 1.62500300 & 0.26989500 \\ \mathrm{C} & -0.84511100 & 2.09352000 & 0.40062400 \\ \mathrm{C} & -2.11137100 & 2.67799600 & 0.56393100 \\ \mathrm{C} & -2.22838800 & 4.00580500 & 1.04742300 \\ \mathrm{C} & -3.47430600 & 4.58283700 & 1.23526200 \\ \mathrm{C} & -3.30479700 & 1.96389000 & 0.25206300 \\ \mathrm{C} & -4.64300200 & 3.87499500 & 0.90632400 \\ \mathrm{C} & -4.55590500 & 2.58218600 & 0.41068600 \\ \mathrm{~N} & -3.12710000 & 0.63632700 & -0.17791800 \\ \mathrm{~N} & -4.15031300 & 0.09170600 & -0.68133100 \\ \mathrm{H} & -1.32233100 & 4.55806500 & 1.27495700 \\ \mathrm{H} & -3.54431200 & 5.59568100 & 1.62257800 \\ \mathrm{H} & -5.61547700 & 4.34116100 & 1.03818300 \\ \mathrm{H} & -5.44256900 & 2.01478900 & 0.15063400 \\ \mathrm{C} & 4.40003900 & 0.38028000 & -0.64075600 \\ \mathrm{C} & 4.01508100 & 1.12622500 & -1.75963900 \\ \mathrm{C} & 5.74380600 & 0.30647500 & -0.25654600 \\ \mathrm{C} & 4.98941500 & 1.81473400 & -2.48176700 \\ \mathrm{H} & 2.97384600 & 1.17159200 & -2.04725400 \\ \mathrm{H} & 6.70529500 & 0.99285500 & -0.99308200 \\ \mathrm{H} & -3.01604200 & -0.28363900 & 0.61069300 \\ \mathrm{H} & -3.33151100 & 1.75187900 & -2.10478900 \\ \mathrm{C} & -1.81904800 & -3.88979700 & -1.18164300 \\ \mathrm{H} & -3.99278800 & 2.39558400 & -3.35027700 \\ \mathrm{H} & 7.74776100 & 0.93847400 & -0.69269400 \\ \mathrm{H} & 7.08379200 & 2.28888300 & -2.67576600 \\ \mathrm{C} & -3.97875200 & -1.26404500 & -1.05176000 \\ \mathrm{C} & -2.78660000 & -1.99498800 & -0.89699200 \\ \mathrm{C} & -5.10684800 & -1.88055400 & -1.61154700 \\ \mathrm{C} & -2.73964200 & -3.32425200 & -1.29996200 \\ \mathrm{H} & -1.92088200 & -1.50439000 & -0.46649000 \\ \mathrm{H} & -5.05247400 & -3.21419300 & -2.01203800 \\ \mathrm{H} & -6.01245100 & -1.29146900 & -1.72073100 \\ \mathrm{H} & -3.86904500 & -3.93852200 & -1.85690700 \\ \mathrm{H} & -37851100 & -2.16888400\end{array}$

29c

Point Group: C1

B3LYP/6-31G* $=-1296.62906 \mathrm{au}$ 
B3LYP/6-31G* Zero Point Corrected Energy = -1296.24593 au NIMAG $=1$

\begin{tabular}{|c|c|c|c|}
\hline \multicolumn{4}{|c|}{ B3LYP/6-31G* Optimized Cartesian Coordinates } \\
\hline $\mathrm{C}$ & 1.34341000 & -1.33809200 & -0.14087800 \\
\hline $\mathrm{C}$ & 1.88895500 & -0.10100800 & -0.46083000 \\
\hline $\mathrm{C}$ & 1.40655100 & 1.06543000 & -1.13877300 \\
\hline $\mathrm{C}$ & 0.15839000 & 1.44430200 & -1.66269300 \\
\hline $\mathrm{C}$ & 0.06975600 & 2.68287100 & -2.27763300 \\
\hline $\mathrm{C}$ & 2.52202700 & 1.93506100 & -1.24824300 \\
\hline $\mathrm{C}$ & 1.18961100 & 3.54982600 & -2.38421000 \\
\hline $\mathrm{C}$ & 2.42366700 & 3.19458900 & -1.87542500 \\
\hline $\mathrm{N}$ & 3.62719700 & 1.38923400 & -0.66670500 \\
\hline $\mathrm{N}$ & 3.25068900 & 0.20378600 & -0.21826800 \\
\hline $\mathrm{H}$ & -0.70157800 & 0.78795600 & -1.58023900 \\
\hline $\mathrm{H}$ & -0.88205300 & 3.00585700 & -2.69056600 \\
\hline $\mathrm{H}$ & 1.06691800 & 4.50971500 & -2.87814800 \\
\hline $\mathrm{H}$ & 3.29015900 & 3.84400600 & -1.95077800 \\
\hline $\mathrm{C}$ & 0.03952500 & -1.64324900 & -0.30448600 \\
\hline $\mathrm{C}$ & -1.13092200 & -2.05597500 & -0.38624200 \\
\hline $\mathrm{C}$ & -2.42786800 & -2.59566000 & -0.49716800 \\
\hline $\mathrm{C}$ & -2.61857100 & -3.97186700 & -0.77402100 \\
\hline $\mathrm{C}$ & -3.89145600 & -4.50214300 & -0.93477700 \\
\hline $\mathrm{C}$ & -3.58187400 & -1.78113300 & -0.35935500 \\
\hline $\mathrm{C}$ & -5.01366300 & -3.67910500 & -0.78621000 \\
\hline $\mathrm{C}$ & -4.85815000 & -2.32330500 & -0.49817400 \\
\hline $\mathrm{N}$ & -3.43872100 & -0.37440300 & -0.16780400 \\
\hline $\mathrm{N}$ & -3.33952200 & -0.02082200 & 1.03011900 \\
\hline $\mathrm{H}$ & -1.73885000 & -4.60088300 & -0.86631900 \\
\hline $\mathrm{H}$ & -4.01453000 & -5.55736200 & -1.16088700 \\
\hline $\mathrm{H}$ & -6.01246500 & -4.09402200 & -0.88988400 \\
\hline $\mathrm{H}$ & -5.72186100 & -1.67483400 & -0.38638600 \\
\hline $\mathrm{C}$ & 4.23267400 & -0.61901000 & 0.41506600 \\
\hline $\mathrm{C}$ & 3.89439200 & -1.45532200 & 1.48426000 \\
\hline $\mathrm{C}$ & 5.55222400 & -0.54768300 & -0.04685200 \\
\hline $\mathrm{C}$ & 4.88828400 & -2.23510300 & 2.07529800 \\
\hline $\mathrm{H}$ & 2.87211000 & -1.49900300 & 1.83306500 \\
\hline $\mathrm{C}$ & 6.53438200 & -1.32555300 & 0.55984500 \\
\hline $\mathrm{H}$ & 5.78910200 & 0.11302400 & -0.87249000 \\
\hline $\mathrm{C}$ & 6.20570100 & -2.17451500 & 1.61958800 \\
\hline $\mathrm{H}$ & 4.62695300 & -2.88636600 & 2.90441300 \\
\hline $\mathrm{H}$ & 7.55739200 & -1.27193600 & 0.19837800 \\
\hline $\mathrm{H}$ & 6.97377900 & -2.78280600 & 2.08918400 \\
\hline $\mathrm{C}$ & -3.21897300 & 1.38539500 & 1.23331600 \\
\hline $\mathrm{C}$ & -3.32474600 & 2.35157600 & 0.22040900 \\
\hline $\mathrm{C}$ & -2.99718500 & 1.77440400 & 2.55892900 \\
\hline $\mathrm{C}$ & -3.20080600 & 3.69747000 & 0.54502300 \\
\hline $\mathrm{H}$ & -3.50907300 & 2.02831900 & -0.79837100 \\
\hline $\mathrm{C}$ & -2.86795700 & 3.12584100 & 2.87713500 \\
\hline $\mathrm{H}$ & -2.92628800 & 1.00118300 & 3.31770700 \\
\hline $\mathrm{C}$ & -2.97024700 & 4.08732900 & 1.87082500 \\
\hline $\mathrm{H}$ & -3.28500600 & 4.45125500 & -0.23320300 \\
\hline
\end{tabular}




$\begin{array}{llll}\mathrm{H} & -2.69082700 & 3.42728900 & 3.90555700 \\ \mathrm{H} & -2.87417600 & 5.14179900 & 2.11523000\end{array}$

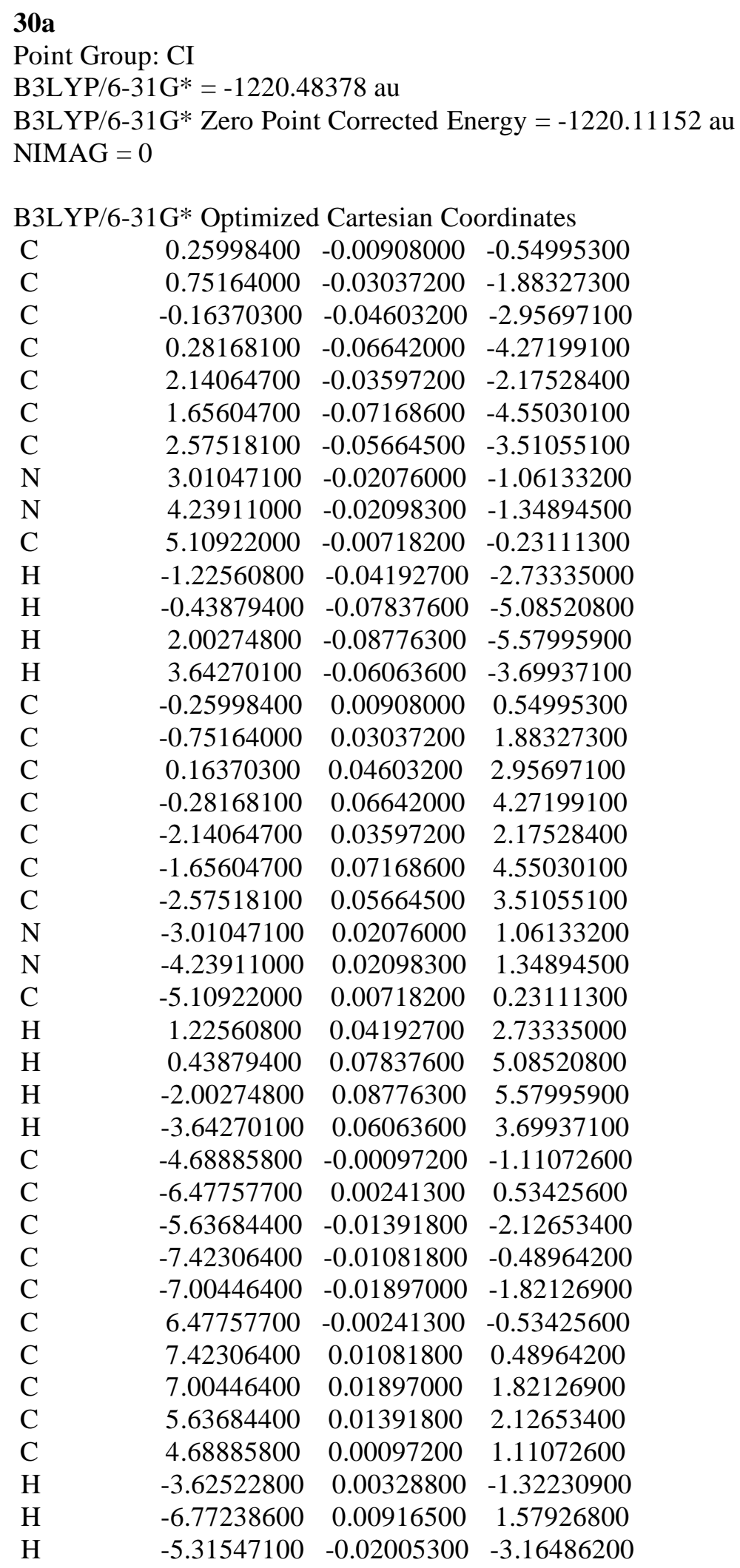




$\begin{array}{lrrr}\mathrm{H} & -8.48271200 & -0.01461500 & -0.24988600 \\ \mathrm{H} & -7.73853300 & -0.02907600 & -2.62250900 \\ \mathrm{H} & 6.77238600 & -0.00916500 & -1.57926800 \\ \mathrm{H} & 8.48271200 & 0.01461500 & 0.24988600 \\ \mathrm{H} & 7.73853300 & 0.02907600 & 2.62250900 \\ \mathrm{H} & 5.31547100 & 0.02005300 & 3.16486200 \\ \mathrm{H} & 3.62522800 & -0.00328800 & 1.32230900\end{array}$

\begin{tabular}{lrrr} 
30b & & & \\
Point Group: CI & & \\
B3LYP/6-31G* $=-1220.47645$ au \\
B3LYP/6-31G* Zero Point Corrected Energy $=-1220.104$ \\
NIMAG = 0 \\
\multicolumn{4}{c}{} \\
B3LYP/6-31G* Optimized Cartesian Coordinates \\
$\mathrm{C}$ & 0.43960200 & 0.39872700 & 0.13708300 \\
$\mathrm{C}$ & 1.29349200 & 1.48113900 & 0.47581900 \\
$\mathrm{C}$ & 0.73523800 & 2.77751600 & 0.47967100 \\
$\mathrm{C}$ & 1.48078800 & 3.89212700 & 0.84817200 \\
$\mathrm{C}$ & 2.65763200 & 1.34292100 & 0.86488200 \\
$\mathrm{C}$ & 2.81136600 & 3.74324200 & 1.25194200 \\
$\mathrm{C}$ & 3.37937900 & 2.47499600 & 1.27253700 \\
$\mathrm{~N}$ & 3.39743900 & 0.14286800 & 0.93141200 \\
$\mathrm{~N}$ & 2.97289600 & -0.80561600 & 0.21766700 \\
$\mathrm{C}$ & 3.71745300 & -2.00800700 & 0.33461200 \\
$\mathrm{H}$ & -0.30426800 & 2.88482000 & 0.18645900 \\
$\mathrm{H}$ & 1.01919400 & 4.87562500 & 0.83337300 \\
$\mathrm{H}$ & 3.39584800 & 4.60753800 & 1.55375600 \\
$\mathrm{H}$ & 4.40658900 & 2.32182700 & 1.58853800 \\
$\mathrm{C}$ & -0.43960200 & -0.39872700 & -0.13708300 \\
$\mathrm{C}$ & -1.29349200 & -1.48113900 & -0.47581900 \\
$\mathrm{C}$ & -0.73523800 & -2.77751600 & -0.47967100 \\
$\mathrm{C}$ & -1.48078800 & -3.89212700 & -0.84817200 \\
$\mathrm{C}$ & -2.65763200 & -1.34292100 & -0.86488200 \\
$\mathrm{C}$ & -2.81136600 & -3.74324200 & -1.25194200 \\
$\mathrm{C}$ & -3.37937900 & -2.47499600 & -1.27253700 \\
$\mathrm{~N}$ & -3.39743900 & -0.14286800 & -0.93141200 \\
$\mathrm{~N}$ & -2.97289600 & 0.80561600 & -0.21766700 \\
$\mathrm{C}$ & -3.71745300 & 2.00800700 & -0.33461200 \\
$\mathrm{H}$ & 0.30426800 & -2.88482000 & -0.18645900 \\
$\mathrm{H}$ & -1.01919400 & -4.87562500 & -0.83337300 \\
$\mathrm{H}$ & -3.39584800 & -4.60753800 & -1.55375600 \\
$\mathrm{H}$ & -4.40658900 & -2.32182700 & -1.58853800 \\
$\mathrm{C}$ & -3.41083400 & 2.99986300 & 0.60733600 \\
$\mathrm{C}$ & -4.69383200 & 2.25127500 & -1.31581200 \\
$\mathrm{C}$ & -4.08011700 & 4.22246300 & 0.58077000 \\
$\mathrm{C}$ & -5.35209100 & 3.47548800 & -1.34085600 \\
$\mathrm{C}$ & -5.05018300 & 4.46279500 & -0.39413300 \\
$\mathrm{C}$ & 4.69383200 & -2.25127500 & 1.31581200 \\
$\mathrm{C}$ & 5.35209100 & -3.47548800 & 1.34085600 \\
$\mathrm{C}$ & 5.05018300 & -4.46279500 & 0.39413300
\end{tabular}




$\begin{array}{lrrr}\mathrm{C} & 4.08011700 & -4.22246300 & -0.58077000 \\ \mathrm{C} & 3.41083400 & -2.99986300 & -0.60733600 \\ \mathrm{H} & -2.65441600 & 2.78215600 & 1.35551900 \\ \mathrm{H} & -4.91286100 & 1.47452000 & -2.04007200 \\ \mathrm{H} & -3.84482400 & 4.98589900 & 1.31718700 \\ \mathrm{H} & -6.10396600 & 3.66813000 & -2.10176100 \\ \mathrm{H} & -5.56907100 & 5.41723000 & -0.42197500 \\ \mathrm{H} & 4.91286100 & -1.47452000 & 2.04007200 \\ \mathrm{H} & 6.10396600 & -3.66813000 & 2.10176100 \\ \mathrm{H} & 5.56907100 & -5.41723000 & 0.42197500 \\ \mathrm{H} & 3.84482400 & -4.98589900 & -1.31718700 \\ \mathrm{H} & 2.65441600 & -2.78215600 & -1.35551900\end{array}$

\section{0c}

Point Group: $\mathrm{C} 1$

B3LYP/6-31G* $=-1220.48039$ au

B3LYP/6-31G* Zero Point Corrected Energy $=-1220.10821 \mathrm{au}$ $\mathrm{NIMAG}=0$

$\begin{array}{lrrr}\text { B3LYP/6-31G* Optimized Cartesian Coordinates } \\ \text { C } & -0.01369000 & -0.36379200 & -0.41276700 \\ \mathrm{C} & -0.41900400 & -1.72258200 & -0.49475100 \\ \mathrm{C} & 0.55154900 & -2.71936900 & -0.73276700 \\ \mathrm{C} & 0.18697100 & -4.05587600 & -0.83151100 \\ \mathrm{C} & -1.77584000 & -2.11399500 & -0.35471700 \\ \mathrm{C} & -1.15667500 & -4.43292000 & -0.69220500 \\ \mathrm{C} & -2.12758700 & -3.46982600 & -0.45486300 \\ \mathrm{~N} & -2.69754400 & -1.07362700 & -0.10029400 \\ \mathrm{~N} & -3.90536100 & -1.43501900 & -0.04697600 \\ \mathrm{C} & -4.82221600 & -0.39217100 & 0.23508000 \\ \mathrm{H} & 1.58901000 & -2.41896000 & -0.83875200 \\ \mathrm{H} & 0.94779700 & -4.80950900 & -1.01547900 \\ \mathrm{H} & -1.43876500 & -5.47963000 & -0.76663100 \\ \mathrm{H} & -3.17205800 & -3.73643400 & -0.33905600 \\ \mathrm{C} & 0.46578900 & 0.75401400 & -0.35713000 \\ \mathrm{C} & 0.84333800 & 2.12320400 & -0.34630900 \\ \mathrm{C} & -0.17743800 & 3.08478900 & -0.50275700 \\ \mathrm{C} & 0.10237800 & 4.44619200 & -0.55374000 \\ \mathrm{C} & 2.18647600 & 2.58974000 & -0.24317400 \\ \mathrm{C} & 1.42439600 & 4.89327400 & -0.46746000 \\ \mathrm{C} & 2.44951600 & 3.96544300 & -0.32852100 \\ \mathrm{~N} & 3.34432000 & 1.79751300 & -0.09628000 \\ \mathrm{~N} & 3.15722000 & 0.62577500 & 0.32959200 \\ \mathrm{C} & 4.33293400 & -0.16165000 & 0.43046900 \\ \mathrm{H} & -1.19998700 & 2.73184500 & -0.59393800 \\ \mathrm{H} & -0.71024300 & 5.15733500 & -0.67354900 \\ \mathrm{H} & 1.65165800 & 5.95421600 & -0.51612600 \\ \mathrm{H} & 3.48784200 & 4.27643200 & -0.26884500 \\ \mathrm{C} & 4.16320300 & -1.41792000 & 1.02903200 \\ \mathrm{C} & 5.60215800 & 0.23012500 & -0.02950400 \\ \mathrm{C} & 5.25142800 & -2.27665800 & 1.17615400\end{array}$




$\begin{array}{lrrr}\mathrm{C} & 6.68183300 & -0.63286900 & 0.11707100 \\ \mathrm{C} & 6.51172800 & -1.88603200 & 0.71994600 \\ \mathrm{C} & -4.45792600 & 0.92766600 & 0.55583700 \\ \mathrm{C} & -5.44701900 & 1.86642100 & 0.82349500 \\ \mathrm{C} & -6.80008800 & 1.50576900 & 0.77400100 \\ \mathrm{C} & -7.16273100 & 0.19514300 & 0.45856500 \\ \mathrm{C} & -6.17588900 & -0.75283400 & 0.19389900 \\ \mathrm{H} & 3.17113800 & -1.69267700 & 1.37469000 \\ \mathrm{H} & 5.71313800 & 1.20365000 & -0.49395700 \\ \mathrm{H} & 5.11684300 & -3.24760900 & 1.64480600 \\ \mathrm{H} & 7.66382300 & -0.33380600 & -0.24023400 \\ \mathrm{H} & 7.36151000 & -2.55451000 & 0.82951600 \\ \mathrm{H} & -3.40471500 & 1.18326400 & 0.59365300 \\ \mathrm{H} & -5.16878600 & 2.88631700 & 1.07618800 \\ \mathrm{H} & -7.56658200 & 2.24653400 & 0.98555300 \\ \mathrm{H} & -8.21101600 & -0.08800300 & 0.42219200 \\ \mathrm{H} & -6.42660800 & -1.78064700 & -0.05055600\end{array}$

\section{1b}

Point Group: C2

B3LYP/6-31G* $=-1220.45772 \mathrm{au}$

B3LYP/6-31G* Zero Point Corrected Energy $=-1220.08684 \mathrm{au}$

NIMAG $=1$

\begin{tabular}{lrrr}
\multicolumn{4}{c}{ B3LYP/6-31G* Optimized Cartesian Coordinates } \\
$\mathrm{C}$ & 1.30505900 & 1.47222500 & 1.12484800 \\
$\mathrm{C}$ & 2.46024700 & 1.11564900 & 1.85992900 \\
$\mathrm{C}$ & 3.43718500 & 2.06377900 & 2.11807200 \\
$\mathrm{C}$ & 1.19470300 & 2.81659800 & 0.66791200 \\
$\mathrm{C}$ & 3.31254800 & 3.39249800 & 1.65948600 \\
$\mathrm{C}$ & 2.19125600 & 3.76858900 & 0.94060300 \\
$\mathrm{~N}$ & 0.04946500 & 3.16043600 & -0.02419900 \\
$\mathrm{~N}$ & -0.65394100 & 2.13208700 & -0.27355500 \\
$\mathrm{C}$ & -1.92235700 & 2.31776600 & -0.85884600 \\
$\mathrm{H}$ & 2.56272200 & 0.09646400 & 2.21876200 \\
$\mathrm{H}$ & 4.31733700 & 1.77580800 & 2.68714900 \\
$\mathrm{H}$ & 4.09418700 & 4.11519800 & 1.87343600 \\
$\mathrm{H}$ & 2.05671100 & 4.78148900 & 0.57329700 \\
$\mathrm{C}$ & -1.30505900 & -1.47222500 & 1.12484800 \\
$\mathrm{C}$ & -2.46024700 & -1.11564900 & 1.85992900 \\
$\mathrm{C}$ & -3.43718500 & -2.06377900 & 2.11807200 \\
$\mathrm{C}$ & -1.19470300 & -2.81659800 & 0.66791200 \\
$\mathrm{C}$ & -3.31254800 & -3.39249800 & 1.65948600 \\
$\mathrm{C}$ & -2.19125600 & -3.76858900 & 0.94060300 \\
$\mathrm{~N}$ & -0.04946500 & -3.16043600 & -0.02419900 \\
$\mathrm{~N}$ & 0.65394100 & -2.13208700 & -0.27355500 \\
$\mathrm{C}$ & 1.92235700 & -2.31776600 & -0.85884600 \\
$\mathrm{H}$ & -2.56272200 & -0.09646400 & 2.21876200 \\
$\mathrm{H}$ & -4.31733700 & -1.77580800 & 2.68714900 \\
$\mathrm{H}$ & -4.09418700 & -4.11519800 & 1.87343600 \\
$\mathrm{H}$ & -2.05671100 & -4.78148900 & 0.57329700
\end{tabular}




$\begin{array}{lrrr}\mathrm{C} & 2.55675800 & -3.56994400 & -0.93558400 \\ \mathrm{C} & 2.55385200 & -1.17602900 & -1.37440000 \\ \mathrm{C} & 3.80863700 & -3.66852200 & -1.53296500 \\ \mathrm{C} & 3.80863700 & -1.28741100 & -1.96907800 \\ \mathrm{C} & 4.43887500 & -2.53114900 & -2.05096800 \\ \mathrm{C} & -2.55385200 & 1.17602900 & -1.37440000 \\ \mathrm{C} & -3.80863700 & 1.28741100 & -1.96907800 \\ \mathrm{C} & -4.43887500 & 2.53114900 & -2.05096800 \\ \mathrm{C} & -3.80863700 & 3.66852200 & -1.53296500 \\ \mathrm{C} & -2.55675800 & 3.56994400 & -0.93558400 \\ \mathrm{H} & 2.05498200 & -4.43942500 & -0.52587100 \\ \mathrm{H} & 2.04707200 & -0.21992400 & -1.30594200 \\ \mathrm{H} & 4.30052300 & -4.63586800 & -1.59259000 \\ \mathrm{H} & 4.29409700 & -0.40201800 & -2.36987500 \\ \mathrm{H} & 5.41837700 & -2.61606500 & -2.51356800 \\ \mathrm{H} & -2.04707200 & 0.21992400 & -1.30594200 \\ \mathrm{H} & -4.29409700 & 0.40201800 & -2.36987500 \\ \mathrm{H} & -5.41837700 & 2.61606500 & -2.51356800 \\ \mathrm{H} & -4.30052300 & 4.63586800 & -1.59259000 \\ \mathrm{H} & -2.05498200 & 4.43942500 & -0.52587100 \\ \mathrm{C} & 0.24234900 & 0.59137300 & 0.85757100 \\ \mathrm{C} & -0.24234900 & -0.59137300 & 0.85757100\end{array}$

\section{$31 \mathrm{c}$}

Point Group: $\mathrm{C} 1$

B3LYP/6-31G* $=-1220.45134$ au

B3LYP/6-31G* Zero Point Corrected Energy $=-1220.08022$ au $\mathrm{NIMAG}=1$

B3LYP/6-31G* Optimized Cartesian Coordinates

$\begin{array}{lrrr}\mathrm{C} & -2.54697500 & -1.28085200 & -0.98871000 \\ \mathrm{C} & -2.74816500 & -2.10455700 & -2.10215000 \\ \mathrm{C} & -3.74040300 & -3.07913100 & -2.04324600 \\ \mathrm{C} & -3.35631200 & -1.45831300 & 0.15314900 \\ \mathrm{C} & -4.53814300 & -3.25100100 & -0.89366100 \\ \mathrm{C} & -4.35747800 & -2.43662600 & 0.21559000 \\ \mathrm{~N} & -3.08928700 & -0.55178800 & 1.17087500 \\ \mathrm{~N} & -2.05199300 & 0.11623800 & 0.85462100 \\ \mathrm{H} & -2.13705500 & -1.96910500 & -2.98901400 \\ \mathrm{H} & -3.90785100 & -3.72003800 & -2.90461300 \\ \mathrm{H} & -5.30072600 & -4.02396800 & -0.88078300 \\ \mathrm{H} & -4.95728200 & -2.54264300 & 1.11416700 \\ \mathrm{C} & -1.60807300 & -0.20232000 & -0.83186400 \\ \mathrm{C} & -0.75541300 & 0.45941900 & -1.52177400 \\ \mathrm{C} & 0.19809100 & 1.44273700 & -1.79942400 \\ \mathrm{C} & -0.19253100 & 2.63481900 & -2.47878000 \\ \mathrm{C} & 0.73683000 & 3.60177000 & -2.80818900 \\ \mathrm{C} & 1.58798300 & 1.28039500 & -1.49723700 \\ \mathrm{C} & 2.10611800 & 3.41946400 & -2.52322400 \\ \mathrm{C} & 2.51920300 & 2.26548700 & -1.88383900 \\ \mathrm{~N} & 1.91665800 & 0.11593700 & -0.79447500\end{array}$




$\begin{array}{lrrr}\mathrm{N} & 3.15411200 & -0.09441900 & -0.61314500 \\ \mathrm{H} & -1.24022200 & 2.77060500 & -2.72840700 \\ \mathrm{H} & 0.40577400 & 4.50803500 & -3.30943700 \\ \mathrm{H} & 2.82879700 & 4.17876500 & -2.80796300 \\ \mathrm{H} & 3.56317100 & 2.09036000 & -1.64778000 \\ \mathrm{C} & 3.45103300 & -1.26254200 & 0.12708500 \\ \mathrm{C} & 2.49787200 & -2.19082400 & 0.58607100 \\ \mathrm{C} & 4.81109800 & -1.47928000 & 0.39425300 \\ \mathrm{C} & 2.91185500 & -3.30766600 & 1.30278500 \\ \mathrm{H} & 1.45068100 & -2.01789500 & 0.36430300 \\ \mathrm{C} & 5.21850400 & -2.59979800 & 1.11551800 \\ \mathrm{H} & 5.52748500 & -0.75146000 & 0.02495500 \\ \mathrm{C} & 4.27031600 & -3.51720200 & 1.57258300 \\ \mathrm{H} & 2.17424100 & -4.02476800 & 1.65453100 \\ \mathrm{H} & 6.27413900 & -2.75828300 & 1.31940200 \\ \mathrm{H} & 4.58468100 & -4.39344400 & 2.13337800 \\ \mathrm{C} & -1.63112500 & 1.19463900 & 1.66316600 \\ \mathrm{C} & -2.54056200 & 1.90062300 & 2.46514700 \\ \mathrm{C} & -0.27273000 & 1.53630300 & 1.64214000 \\ \mathrm{C} & -2.08038800 & 2.95229600 & 3.24975000 \\ \mathrm{H} & -3.58710100 & 1.61605500 & 2.45601000 \\ \mathrm{C} & 0.17240400 & 2.59315800 & 2.43413300 \\ \mathrm{H} & 0.41712600 & 0.96136600 & 1.03436600 \\ \mathrm{C} & -0.72561100 & 3.30258000 & 3.23399800 \\ \mathrm{H} & -2.77908500 & 3.50546500 & 3.87115100 \\ \mathrm{H} & 1.22520300 & 2.85913500 & 2.42567500 \\ \mathrm{H} & -0.37280700 & 4.12856400 & 3.84529900\end{array}$

$32 \mathrm{c}$

Point Group: C1

B3LYP/6-31G* $=-1220.46081 \mathrm{au}$

B3LYP/6-31G* Zero Point Corrected Energy $=-1220.08760 \mathrm{au}$ NIMAG $=0$

B3LYP/6-31G* Optimized Cartesian Coordinates

$\begin{array}{lrrr}\mathrm{C} & -2.56162400 & 1.71482600 & 0.52611600 \\ \mathrm{C} & -2.59414300 & 2.83918800 & 1.36322300 \\ \mathrm{C} & -3.61741500 & 3.75335800 & 1.17116100 \\ \mathrm{C} & -3.55216000 & 1.53453300 & -0.46981300 \\ \mathrm{C} & -4.61219200 & 3.56618900 & 0.17369100 \\ \mathrm{C} & -4.59663600 & 2.46396400 & -0.65846700 \\ \mathrm{~N} & -3.31165700 & 0.40237700 & -1.19377400 \\ \mathrm{~N} & -2.25722700 & -0.16817200 & -0.65099200 \\ \mathrm{H} & -1.83180400 & 2.97367100 & 2.12399100 \\ \mathrm{H} & -3.66457600 & 4.64218000 & 1.79447000 \\ \mathrm{H} & -5.39667800 & 4.30998500 & 0.06768700 \\ \mathrm{H} & -5.34883700 & 2.30457700 & -1.42436100 \\ \mathrm{C} & -1.69560000 & 0.57108500 & 0.45038800 \\ \mathrm{C} & -0.66775600 & 0.26631400 & 1.29999300 \\ \mathrm{C} & -0.05005600 & -0.95897000 & 1.62898900 \\ \mathrm{C} & -0.78295200 & -2.00620600 & 2.26289300\end{array}$




$\begin{array}{lrrr}\mathrm{C} & -0.13436900 & -3.12706500 & 2.74906000 \\ \mathrm{C} & 1.35969000 & -1.13942000 & 1.48305500 \\ \mathrm{C} & 1.25672200 & -3.28981700 & 2.59107500 \\ \mathrm{C} & 1.99151500 & -2.30545500 & 1.95111200 \\ \mathrm{~N} & 2.00405000 & -0.07870400 & 0.84409000 \\ \mathrm{~N} & 3.18413000 & -0.29458900 & 0.43612300 \\ \mathrm{H} & -1.85612000 & -1.89610000 & 2.38731100 \\ \mathrm{H} & -0.71366900 & -3.90210600 & 3.24524400 \\ \mathrm{H} & 1.74647700 & -4.18415300 & 2.96530600 \\ \mathrm{H} & 3.06166500 & -2.40159200 & 1.80312800 \\ \mathrm{C} & 3.83166900 & 0.83719500 & -0.11407500 \\ \mathrm{C} & 5.10142800 & 0.60332200 & -0.66192600 \\ \mathrm{C} & 3.29248700 & 2.13686700 & -0.13616600 \\ \mathrm{C} & 5.82502200 & 1.64845000 & -1.23281900 \\ \mathrm{H} & 5.49641600 & -0.40773800 & -0.62792200 \\ \mathrm{C} & 4.02015700 & 3.17410100 & -0.70809600 \\ \mathrm{H} & 2.31242900 & 2.30214000 & 0.29800400 \\ \mathrm{C} & 5.28628500 & 2.93644100 & -1.25835400 \\ \mathrm{H} & 6.80794600 & 1.45922000 & -1.65595300 \\ \mathrm{H} & 3.60389800 & 4.17828300 & -0.72413500 \\ \mathrm{H} & 5.84923600 & 3.75436400 & -1.70015400 \\ \mathrm{C} & -1.73124300 & -1.34246600 & -1.27284400 \\ \mathrm{C} & -2.61587400 & -2.35612200 & -1.65265400 \\ \mathrm{C} & -0.36215500 & -1.44572300 & -1.52783700 \\ \mathrm{C} & -2.11548100 & -3.49474500 & -2.27890500 \\ \mathrm{H} & -3.67573900 & -2.24128100 & -1.45414000 \\ \mathrm{C} & 0.12549400 & -2.59426800 & -2.14947400 \\ \mathrm{H} & 0.30760200 & -0.63859700 & -1.25424900 \\ \mathrm{C} & -0.74534200 & -3.61899600 & -2.52365900 \\ \mathrm{H} & -2.79736500 & -4.28783400 & -2.57191800 \\ \mathrm{H} & 1.18995900 & -2.68000600 & -2.34591900 \\ \mathrm{H} & -0.35873700 & -4.51087700 & -3.00851800\end{array}$

\footnotetext{
$33 c$

Point Group: $\mathrm{C} 1$

B3LYP/6-31G* $=-1220.44440$ au

B3LYP/6-31G* Zero Point Corrected Energy $=-1220.07210 \mathrm{au}$ $\mathrm{NIMAG}=1$

B3LYP/6-31G* Optimized Cartesian Coordinates

$\begin{array}{lrrr}\mathrm{C} & -1.79885300 & 2.25753100 & 0.34367900 \\ \mathrm{C} & -1.27933900 & 3.42936300 & 0.91383900 \\ \mathrm{C} & -2.03788300 & 4.58407700 & 0.81650400 \\ \mathrm{C} & -3.06161300 & 2.27037600 & -0.30300600 \\ \mathrm{C} & -3.30853100 & 4.59134700 & 0.17903300 \\ \mathrm{C} & -3.83582900 & 3.44853200 & -0.38863600 \\ \mathrm{~N} & -3.34532200 & 1.05340100 & -0.84212900 \\ \mathrm{~N} & -2.36562500 & 0.24462500 & -0.46119700 \\ \mathrm{H} & -0.30894800 & 3.41209800 & 1.39961000 \\ \mathrm{H} & -1.65565600 & 5.51314900 & 1.23064600 \\ \mathrm{H} & -3.86993600 & 5.52057000 & 0.13751400\end{array}$
}




$\begin{array}{lrrr}\mathrm{H} & -4.80490700 & 3.43904600 & -0.87730600 \\ \mathrm{C} & -1.32436600 & 0.90416300 & 0.28274700 \\ \mathrm{C} & -0.18385900 & 0.42031900 & 0.87973000 \\ \mathrm{C} & 0.00478200 & -0.91123200 & 1.36332600 \\ \mathrm{C} & -1.00827100 & -1.54563500 & 2.13475400 \\ \mathrm{C} & -0.80398000 & -2.77328300 & 2.75362300 \\ \mathrm{C} & 1.23902100 & -1.61851000 & 1.22273200 \\ \mathrm{C} & 0.39110200 & -3.46692900 & 2.54818500 \\ \mathrm{C} & 1.38682000 & -2.89923300 & 1.74981200 \\ \mathrm{~N} & 2.32649400 & -1.15787600 & 0.41198000 \\ \mathrm{~N} & 2.81488100 & -0.05791200 & 0.76864100 \\ \mathrm{H} & -1.95284800 & -1.02556100 & 2.26432500 \\ \mathrm{H} & -1.59210300 & -3.20854600 & 3.36237900 \\ \mathrm{H} & 0.54598600 & -4.44498000 & 2.99470000 \\ \mathrm{H} & 2.31386200 & -3.43111100 & 1.55457600 \\ \mathrm{C} & 3.89566700 & 0.39832700 & -0.03960400 \\ \mathrm{C} & 4.44386800 & 1.62752400 & 0.34486200 \\ \mathrm{C} & 4.41661600 & -0.28862600 & -1.14859500 \\ \mathrm{C} & 5.50695800 & 2.17482900 & -0.37258600 \\ \mathrm{H} & 4.01583500 & 2.13230500 & 1.20540200 \\ \mathrm{C} & 5.47747000 & 0.26108000 & -1.85888600 \\ \mathrm{H} & 3.97816800 & -1.24029400 & -1.42858900 \\ \mathrm{C} & 6.02469500 & 1.49240900 & -1.47446400 \\ \mathrm{H} & 5.92981700 & 3.12986800 & -0.07343400 \\ \mathrm{H} & 5.88449600 & -0.26709100 & -2.71729300 \\ \mathrm{H} & 6.85346400 & 1.91539300 & -2.03607100 \\ \mathrm{C} & -2.34190000 & -1.06981000 & -1.01958500 \\ \mathrm{C} & -3.53001900 & -1.80647800 & -1.05387700 \\ \mathrm{C} & -1.16224100 & -1.58948000 & -1.55841000 \\ \mathrm{C} & -3.52673400 & -3.08192300 & -1.61277000 \\ \mathrm{H} & -4.43597200 & -1.37210500 & -0.64609400 \\ \mathrm{C} & -1.17056300 & -2.87143900 & -2.10602100 \\ \mathrm{H} & -0.25604900 & -0.99462000 & -1.56074200 \\ \mathrm{C} & -2.34776900 & -3.62082000 & -2.13415400 \\ \mathrm{H} & -4.44786100 & -3.65730100 & -1.63677300 \\ \mathrm{H} & -0.25306400 & -3.27762800 & -2.52162400 \\ \mathrm{H} & -2.34876800 & -4.61772600 & -2.56568600\end{array}$

34

Point Group: C2

B3LYP/6-31G* $=-1220.58501 \mathrm{au}$

B3LYP/6-31G* Zero Point Corrected Energy $=-1220.20679$ au

$\mathrm{NIMAG}=0$

B3LYP/6-31G* Optimized Cartesian Coordinates

$\begin{array}{llll}\mathrm{C} & -1.64710000 & 0.86559900 & -0.97646700 \\ \mathrm{C} & -2.60188500 & 0.08433600 & -1.67400900 \\ \mathrm{C} & -3.73254400 & 0.71346400 & -2.14202200 \\ \mathrm{C} & -1.85995500 & 2.26882400 & -0.78685000 \\ \mathrm{C} & -3.94915500 & 2.11099500 & -1.93965500 \\ \mathrm{C} & -3.03650300 & 2.89492300 & -1.27430800\end{array}$




$\begin{array}{lrrr}\mathrm{N} & -0.83527500 & 2.84188700 & -0.12903200 \\ \mathrm{~N} & 0.02573400 & 1.82507200 & 0.10894200 \\ \mathrm{C} & 1.22903000 & 2.13217600 & 0.81836500 \\ \mathrm{H} & -2.44656200 & -0.97904700 & -1.82844500 \\ \mathrm{H} & -4.48336100 & 0.14012600 & -2.67867700 \\ \mathrm{H} & -4.86053500 & 2.55882100 & -2.32680300 \\ \mathrm{H} & -3.19229300 & 3.95811200 & -1.12036000 \\ \mathrm{C} & 1.64710000 & -0.86559900 & -0.97646700 \\ \mathrm{C} & 2.60188500 & -0.08433600 & -1.67400900 \\ \mathrm{C} & 3.73254400 & -0.71346400 & -2.14202200 \\ \mathrm{C} & 1.85995500 & -2.26882400 & -0.78685000 \\ \mathrm{C} & 3.94915500 & -2.11099500 & -1.93965500 \\ \mathrm{C} & 3.03650300 & -2.89492300 & -1.27430800 \\ \mathrm{~N} & 0.83527500 & -2.84188700 & -0.12903200 \\ \mathrm{~N} & -0.02573400 & -1.82507200 & 0.10894200 \\ \mathrm{C} & -1.22903000 & -2.13217600 & 0.81836500 \\ \mathrm{H} & 2.44656200 & 0.97904700 & -1.82844500 \\ \mathrm{H} & 4.48336100 & -0.14012600 & -2.67867700 \\ \mathrm{H} & 4.86053500 & -2.55882100 & -2.32680300 \\ \mathrm{H} & 3.19229300 & -3.95811200 & -1.12036000 \\ \mathrm{C} & -1.85995500 & -3.35349000 & 0.55951900 \\ \mathrm{C} & -1.74808800 & -1.25418700 & 1.77401300 \\ \mathrm{C} & -3.02165800 & -3.68605000 & 1.25271200 \\ \mathrm{C} & -2.91670600 & -1.59511700 & 2.45449400 \\ \mathrm{C} & -3.55743500 & -2.80766500 & 2.19711200 \\ \mathrm{C} & 1.74808800 & 1.25418700 & 1.77401300 \\ \mathrm{C} & 2.91670600 & 1.59511700 & 2.45449400 \\ \mathrm{C} & 3.55743500 & 2.80766500 & 2.19711200 \\ \mathrm{C} & 3.02165800 & 3.68605000 & 1.25271200 \\ \mathrm{C} & 1.85995500 & 3.35349000 & 0.55951900 \\ \mathrm{H} & -1.42461300 & -4.02654600 & -0.16969300 \\ \mathrm{H} & -1.24638900 & -0.31868500 & 1.99171500 \\ \mathrm{H} & -3.51061800 & -4.63474600 & 1.04996800 \\ \mathrm{H} & -3.31908400 & -0.91023100 & 3.19530200 \\ \mathrm{H} & -4.46575000 & -3.06881900 & 2.73261500 \\ \mathrm{H} & 1.24638900 & 0.31868500 & 1.99171500 \\ \mathrm{H} & 3.31908400 & 0.91023100 & 3.19530200 \\ \mathrm{H} & 4.46575000 & 3.06881900 & 2.73261500 \\ \mathrm{H} & 3.51061800 & 4.63474600 & 1.04996800 \\ \mathrm{H} & 1.42461300 & 4.02654600 & -0.16969300 \\ \mathrm{C} & -0.40047700 & 0.60892200 & -0.38040300 \\ \mathrm{C} & 0.40047700 & -0.60892200 & -0.38040300\end{array}$

